\title{
Inflammatory Disease Processes and Interactions with Nutrition
}

P. C. Calder ${ }^{1}$, R. Albers ${ }^{2}$, J.-M. Antoine ${ }^{3}$, S. Blum ${ }^{4}$, R. Bourdet-Sicard ${ }^{3}$, G. A. Ferns ${ }^{5}$, G. Folkerts ${ }^{6}$, P. S. Friedmann ${ }^{1}$, G. S. Frost ${ }^{7}$, F. Guarner ${ }^{8}$, M. Løvik ${ }^{9,10}$, S. Macfarlane ${ }^{11}$, P. D. Meyer ${ }^{12}$, L. M'Rabet ${ }^{13}$, M. Serafini ${ }^{14}$, W. van $\operatorname{Eden}^{15}$, J. van Loo ${ }^{16}$, W. Vas Dias ${ }^{17}$, S. Vidry ${ }^{18 *}$, B. M. Winklhofer-Roob ${ }^{19}$ and J. Zhao ${ }^{20}$

1. School of Medicine, University of Southampton, Southampton SO16 6YD, UK

2. Unilever Food \& Health Research Institute, Unilever R\&D Vlaardingen, 3130 AC Vlaardingen, The Netherlands

3. Danone Vitapole, 91767 Palaiseau Cedex, France

4. Nutrition \& Health Department, Nestlé Research Center, Vers-chez-les-Blanc, 1000 Lausanne 26, Switzerland

5. Postgraduate Medical School, University of Surrey, Guildford GU2 7WG, UK

6. School of Biomedical and Life Sciences, University of Surrey, Guildford GU2 7XH, UK

7. Department of Pharmacology and Pathophysiology, Utrecht Institute for Pharmaceutical Sciences, University of Utrecht, 3508 TB Utrecht, The Netherlands

8. Digestive System Research Unit, University Hospital Vall d'Hebron, 08035 Barcelona, Spain

9. Division of Environmental Medicine, Norwegian Institute of Public Health, 0403 Oslo, Norway

10. Institute for Cancer Research and Molecular Medicine, NTNU, Trondheim, Norway

11. Division of Pathology and Neuroscience, Ninewells Hospital and Medical School, Dundee University, Dundee DD1 9SY, UK

12. Royal Cosun, 4704 RA Roosendaal, The Netherlands

13. Danone Research - Centre for Specialised Nutrition, 6700 CA Wageningen, The Netherlands

14. Antioxidant Research Laboratory, Unit of Human Nutrition, Istituto Nazionale di Ricerca per gli Alimenti e la Nutrizione, 00178 Rome, Italy

15. Division of Immunology, Faculty of Veterinary Medicine, University of Utrecht, 5384 CL Utrecht, The Netherlands

16. Südzucker/BENEO Group, 3300 Tienen, Belgium

17. Seven Seas Limited, Marfleet, Hull HU9 5NJ, UK

18. International Life Sciences Institute Europe, Av. E. Mounier 83, Box 6 - 1200 Brussels, Belgium

19. Human Nutrition \& Metabolism Research and Training Center, Institute of Molecular Biosciences, Karl-Franzens University, 8010 Graz, Austria

20. Yakult Europe, 1332 EN Alemere, The Netherlands

*Corresponding author: Dr Stéphane Vidry, ILSI Europe

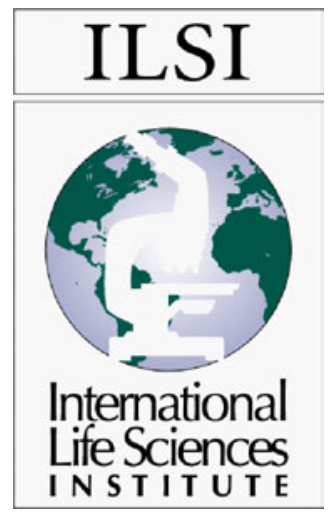

Commissioned by the

ILSI Europe Nutrition and Immunity Task Force

Correspondence: ILSI Europe a.i.s.b.l. - Avenue E. Mounier 83, Box 6 - 1200 Brussels - Belgium Email: publications@ilsieurope.be - Fax: +32 27620044 


\title{
Table of Contents
}

Key words: Inflammation, Cytokine, Eicosanoid, Leucocyte, Antioxidant, Fatty acid, Flavonoids, Prebiotic, Probiotic

Correspondence: ILSI Europe a.i.s.b.1., Avenue E. Mounier 83, Box 6 - 1200 Brussels, Belgium, fax: + 32276200 44, email: publications@ilsieurope.be

\begin{abstract}
Abbreviations: AA, ascorbic acid; AMPK, AMP-activated protein kinase; CCP, cyclic citrullinated peptides; CD, Crohn's disease; CEHC, carboxyethyl hydroxychromans; COPD, chronic obstructive pulmonary disease; COX, cyclo-oxygenase; CRP, C-reactive protein; DC, dendritic cells; EPA, eicosapentaenoic acid; HLA, human leucocyte antigen; IBD, inflammatory bowel disease; IFN, interferon; JIA, juvenile idiopathic arthritis; LTB4, leukotriene B4; LOX, lipoxygenase; NOD, nucleotide-binding oligomerisation domain; PAI-1, plasminogen activation inhibitor 1; RA, rheumatoid arthritis; ROS, reactive oxygen species; STAT, signal transducers and activators of transcription; TAC, total antioxidant capacity; TGF, transforming growth factor; UC, ulcerative colitis.

(C) ILSI Europe
\end{abstract}


Inflammation is a stereotypical physiological response to infections and tissue injury; it initiates pathogen killing as well as tissue repair processes and helps to restore homeostasis at infected or damaged sites. Acute inflammatory reactions are usually self-limiting and resolve rapidly, due to the involvement of negative feedback mechanisms. Thus, regulated inflammatory responses are essential to remain healthy and maintain homeostasis. However, inflammatory responses that fail to regulate themselves can become chronic and contribute to the perpetuation and progression of disease. Characteristics typical of chronic inflammatory responses underlying the pathophysiology of several disorders include loss of barrier function, responsiveness to a normally benign stimulus, infiltration of inflammatory cells into compartments where they are not normally found in such high numbers, and overproduction of oxidants, cytokines, chemokines, eicosanoids and matrix metalloproteinases. The levels of these mediators amplify the inflammatory response, are destructive and contribute to the clinical symptoms. Various dietary components including long chain $\omega-3$ fatty acids, antioxidant vitamins, plant flavonoids, prebiotics and probiotics have the potential to modulate predisposition to chronic inflammatory conditions and may have a role in their therapy. These components act through a variety of mechanisms including decreasing inflammatory mediator production through effects on cell signaling and gene expression ( $\omega-3$ fatty acids, vitamin E, plant flavonoids), reducing the production of damaging oxidants (vitamin $\mathrm{E}$ and other antioxidants), and promoting gut barrier function and anti-inflammatory responses (prebiotics and probiotics). However, in general really strong evidence of benefit to human health through anti-inflammatory actions is lacking for most of these dietary components. Thus, further studies addressing efficacy in humans linked to studies providing greater understanding of the mechanisms of action involved are required.

\section{Preamble}

Inflammation is a normal host defence mechanism that protects the host from infection and other insults; it initiates pathogen killing as well as tissue repair processes and helps to restore homeostasis at infected or damaged sites. It is typed by redness, swelling, heat, pain and loss of function, and involves interactions among many cell types and the production of, and responses to, a number of chemical mediators. Normally, the host is tolerant to microbes and other environmental components that do not pose a threat. This tolerance involves only a limited host response or an active response that is tightly controlled. Where an inflammatory response does occur, it is normally well regulated in order that it does not cause excessive damage to the host, is self-limiting and resolves rapidly. This self-regulation involves the activation of negative feedback mechanisms such as the secretion of anti-inflammatory cytokines, inhibition of pro-inflammatory signalling cascades, shedding of receptors for inflammatory mediators and activation of regulatory cells. As such, and controlled properly, regulated inflammatory responses are essential to remain healthy and maintain homeostasis. Pathological inflammation involves a loss of tolerance and/or of regulatory processes. Where this becomes excessive, irreparable damage to host tissues and disease can occur. Typically, diseases or conditions with a well-recognised inflammatory component are treated with general or specific anti-inflammatory pharmaceuticals. However, since many dietary components may influence various elements of inflammation, nutrition may play a role in predisposing to inflammatory conditions and altered nutrition may be useful in therapy of such conditions. A workshop was held in Oporto, Portugal, on 29-31 May 2006. The workshop aimed to consider the role of inflammation in various diseases and conditions, to identify common and unique mechanisms and markers of inflammation, and to review and consolidate evidence that dietary components can influence inflammatory processes and to understand their mechanisms of action. The present paper is based upon the presentations made at the workshop and the subsequent discussions.

\section{General aspects of the inflammatory process}

Inflammation may be classified into four types, the mechanisms of which in part overlap: (i) inflammation caused by innate and acquired immunity against infectious agents, in which cells are activated and mediators released to prevent or combat infection and remove foreign material; (ii) inflammation caused by different inhaled agents ('irritants') like diesel exhaust particles, ozone and endotoxin; (iii) allergic inflammation, in which specific IgE antibodies bound to mast cells upon cross-linking by allergen cause the immediate release of a number of inflammatory mediators and activation of inflammatory cells; and (iv) neurogenic inflammation, mediated by the neural system. Common to these forms of inflammation is that they have an afferent phase, in which the presence of a 'foreign material' is sensed by some types of cell, and an efferent phase, in which an inflammatory response is generated to eliminate the perceived hostile intruder. The purpose of the inflammatory response to micro-organisms is obvious, and the response is beneficial and necessary to protect the integrity of the body as long as it does not become unnecessarily destructive or long-lasting. Inflammation caused by non-pathogenic agents can also be beneficial and remove the foreign material e.g. by increasing mucous production and increasing the number of phagocytic cells, but it may also have negative health effects in particular if long-lasting. Allergic inflammation is triggered by minute amounts of innocuous foreign material, so-called allergens from plants, insects, animals and foods, and serves no obvious beneficial purpose, except that similar responses may protect against certain infectious agents (parasites). Neurogenic inflammation may be looked upon as an adjuct mechanism to the other three types of inflammation. Irrespective of the cause of the inflammation, the response involves four major events.

(i) An increased blood supply to the site of inflammation.

(ii) Increased capillary permeability caused by retraction of endothelial cells. This permits larger molecules, not normally capable of traversing the endothelium, to do so and thus delivers some soluble mediators to the site of inflammation.

(iii) Leucocyte migration from the capillaries into the surrounding tissue (Fig. 1). This is promoted by release of chemoattractants from the site of inflammation and by the upregulation of adhesion molecules on the endothelium. Once in the tissue the leucocytes move to the site of inflammation.

(iv) Release of mediators from leucocytes at the site of inflammation (Fig. 1). These may include lipid mediators 


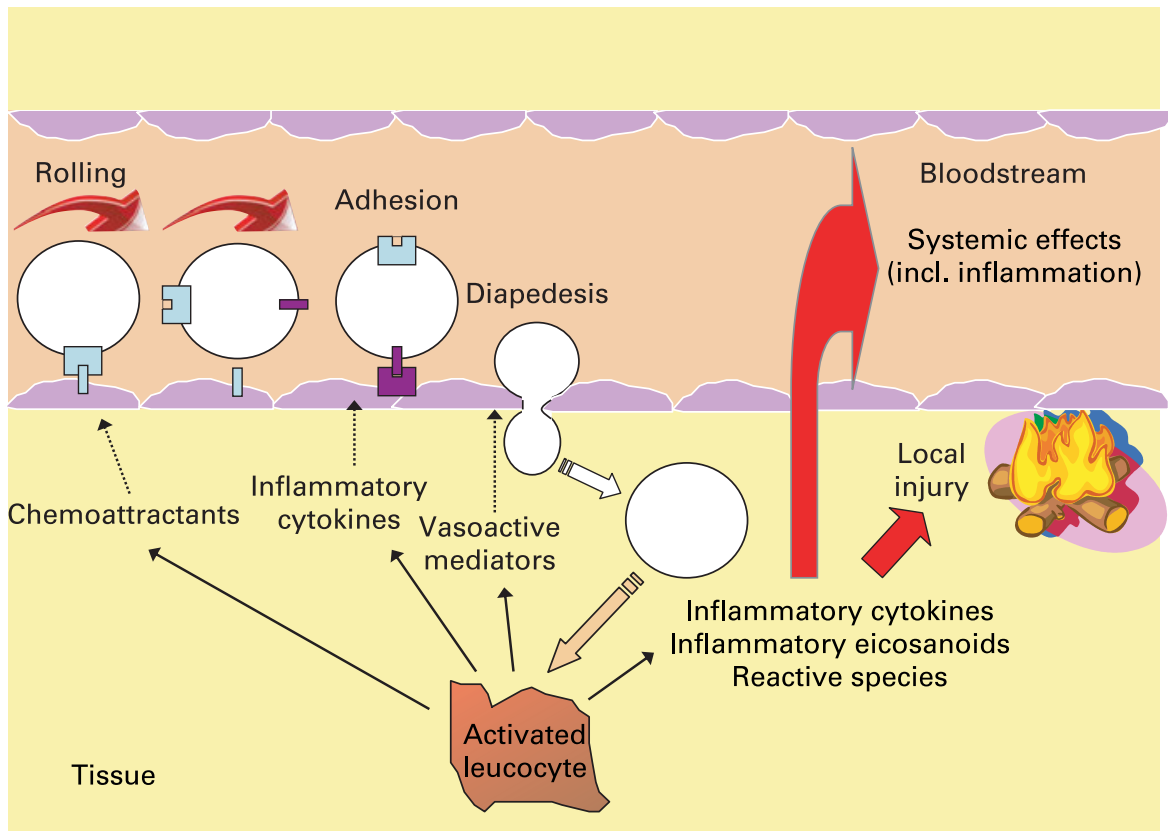

Fig. 1. Generalised view of inflammation

(e.g. PG, leukotrienes), peptide mediators (e.g. cytokines), reactive oxygen species (ROS, e.g. superoxide), amino acid derivatives (e.g. histamine) and enzymes (e.g. matrix proteases) depending upon the cell type involved, the nature of the inflammatory stimulus, the anatomical site involved and the stage during the inflammatory response. These mediators normally would play a role in host defence, but when produced inappropriately or in an unregulated fashion, they can cause damage to host tissues, leading to disease. Several of these mediators may act to amplify the inflammatory process acting, for example, as chemoattractants. Some of the inflammatory mediators may escape the inflammatory site into the circulation and from there they can exert systemic effects. For example, the cytokine IL-6 induces hepatic synthesis of the acute phase protein $\mathrm{C}$-reactive protein (CRP), while the cytokine TNF- $\alpha$ elicits metabolic effects within skeletal muscle, adipose tissue and bone.

\section{Characteristics of inflammatory conditions afflicting specific organ systems}

\section{Gut: celiac disease}

Normal function of mucosal immunity. The gastrointestinal mucosa is an interface for communication between the individual and the external environment. The large mucosal surface $\left(300-400 \mathrm{~m}^{2}\right.$ in an adult human) is adapted with specialised structures for the chemical and immunological recognition of substances and organisms passing through the tract. Intestinal epithelial cells play a crucial role in detecting foreign substances and mediating host innate and adaptive mucosal immune responses. Activation of innate host defence mechanisms is based on the rapid recognition of conserved molecular patterns in microbes by preformed receptors, tolllike receptors, mainly expressed in the cell membrane and nucleotide-binding oligomerisation domain (NOD)-family (also known as caspase recruitment domain-family) receptors in the cytosol ${ }^{(1)}$. In response to invading bacteria, the signals converge to transcription factors (NF- $\mathrm{B}$ ) and others), which initiate the transcription of genes responsible for the synthesis of proinflammatory proteins ${ }^{(2)}$. Intestinal epithelial cells also express human leucocyte antigen (HLA) class II molecules, suggesting that they can function as antigen-presenting cells $^{(3)}$. On the other hand, non-pathogenic bacteria and innocuous food antigens may elicit other types of cytokine responses that are transmitted to underlying immunocompetent cells ${ }^{(4)}$. Normally, responses to non-pathogenic bacteria involve regulatory cytokines such as transforming growth factor (TGF)- $\beta$ or IL-10, and are related with the induction of regulatory pathways of the immune system ${ }^{(5,6)}$. Acquired immune responses develop in specialised lymphoid tissues. Gut-associated lymphoid tissue is located in three compartments: organised structures (Peyer's patches and lymphoid follicles); the lamina propria; the surface epithelium ${ }^{(7)}$. The organised structures are the inductive sites for acquired immunity. These structures are covered by follicle-associated epithelium, which contains M-cells. These are specialised epithelial cells that transport micro-organisms and other antigens from the gut lumen into the organised lymphoid tissue. Antigens are presented to naïve T-cells by antigen-presenting cells such as dendritic cells (DC) and macrophages. In addition, luminal antigens may also be taken up and presented by epithelial cells. After priming, antigen-specific T-cell clones proliferate, but these $\mathrm{T}$-cells may differentiate into Th1, Th2 or regulatory T-cells, with different effector capabilities ${ }^{(8)}$. Th1 or Th2 cells have a polarised cytokine secretion either of interferon (IFN)- $\gamma$, TNF- $\alpha$ and IL-2 (Th1), or IL-4, IL-5 and IL-13 (Th2). Such skewing of the adaptive immune response depends on the presence of microenvironmental factors, including 'danger signals' from microbial products, and eventually induces pro-inflammatory systemic-type immunity, with the potential for tissue damage. By contrast, regulatory T-cells produce IL-10 and 
TGF- $\beta$ and lead to suppressive mechanisms that avoid local and peripheral hypersensitivity to innocuous antigens in food. Such mucosal tolerance to food antigens is an important adaptive immune function in view of the fact that as much as a ton of food may pass through the gut of an adult every year, resulting in substantial exposure to antigens. Thus, in the healthy state, the vulnerable gut mucosa exhibits virtually no proinflammatory response to food antigens ${ }^{(9,10)}$ and contains very few hyperactivated T-cells.

Definition and description of celiac disease. Celiac disease is an immune-mediated disorder that affects primarily the small intestinal mucosa. The disease is triggered by the ingestion of gluten in genetically susceptible individuals. Strictly speaking, gluten is a protein component in wheat, but the term is collectively applied to disease-activating proteins in wheat, rye and barley. Celiac disease is characterised by chronic inflammation of the small intestinal mucosa that may result in atrophy of intestinal villi. The progressive destruction of the small intestinal mucosa causes malabsorption, and a variety of clinical manifestations, including diarrhoea, abdominal pain, vitamin and mineral deficiencies, iron-deficiency anaemia, osteoporosis, growth delay, skin lesions, neurological disorders, etc. Diagnosis of the disease requires examination of biopsies of small intestinal mucosa ${ }^{(11)}$. The Marsh classification ${ }^{(12)}$ has been adopted to describe the progression of the abnormalities in the mucosa, from early stages with normal architecture and a lymphocytic infiltration of the villus epithelial layer up to total atrophy of the villi caused by chronic inflammation. A number of serologic tests are available commercially for identifying individuals who require an intestinal biopsy examination to diagnose celiac disease ${ }^{(13)}$. The best markers are the detection in serum of anti-tissue transglutaminase IgA by ELISA, or anti-endomysial IgA by immunofluorescence. Both tests appear to have equivalent diagnostic accuracy as the tissue transglutaminase is the specific protein that is recognised by the IgA-endomysial antibody. Antigliadin antibody tests are no longer routinely recommended because of their lower sensitivity and specificity ${ }^{(13)}$. The increasing use of serologic screening is leading to diagnosis in milder cases. It is presently recognised that, at certain points in time, the disease is not associated with obvious clinical signs and symptoms. Silent celiac disease is characterised by the presence of positive serologic markers (anti-tissue transglutaminase and/or endomysial antibody) and histological lesions on small bowel biopsy typical for celiac disease in an asymptomatic individual. In latent celiac disease, small bowel biopsy shows only minimal changes (increased intraepithelial lymphocyte infiltration) and anti-tissue transglutaminase or endomysial antibodies may be detected, but the characteristic feature is that the subjects develop symptoms and positive serologic and histological markers, while on a gluten-containing diet. Latent celiac disease precedes diagnosis of celiac disease or follows successful treatment of active disease with a gluten-free diet. Finally, active celiac disease is characterised by intestinal and/or extraintestinal symptoms, villus atrophy and strongly positive anti-tissue transglutaminase and endomysial antibodies.

Epidemiology of celiac disease. Population-based estimates of the incidence of confirmed celiac disease vary from two to thirteen new cases per 100000 person-years.
These rates have to be interpreted with caution because many patients diagnosed as adults are likely to have had several years of untreated celiac disease, and thus do not represent true new cases. The recent increase in the incidence rates has likely been due to increasing use of serologic screening leading to diagnosis in milder cases ${ }^{(14)}$. Although the prevalence of diagnosed celiac disease varies widely among different populations, the estimates of combined undiagnosed and diagnosed (latent, silent and active) celiac disease are remarkably similar, between $0.7 \%$ and $2.0 \%$, in most European and non-European white populations, including those in Finland, Sweden, Germany, Italy, Spain, Israel, the United States, Argentina, Australia and New Zealand ${ }^{(14,15)}$. However, celiac disease is virtually unknown in East Asian and African populations, whereas rates close to those in Europe have been reported from the Middle East and India ${ }^{(14)}$.

Genetic background appears to be a major risk factor for celiac disease. It is unequivocal that celiac disease is strongly associated with specific HLA class II genes that map to the DQ locus of the MHC used for cell-to-cell interaction during the process of antigen presentation ${ }^{(16)}$. The presence of specific alleles at the HLA-DQ locus appears to be necessary, although not sufficient, for the phenotypic expression of disease. Thus, virtually all affected individuals have either DQ2 (HLA-DQA1*05-DQB1*02) or DQ8 (HLADQA1*03-DQB1*0302) alleles, in comparison with the general population in which $39.5 \%$ have either DQ2 or DQ8. However, only $3 \%$ of individuals in the general population carrying DQ2 or DQ8 will develop evidence of celiac disease, suggesting that HLA typing could be used to identify genetic risk, but not for defining celiac disease ${ }^{(16)}$.

Environmental factors also play a major role. It is well established that celiac disease is strictly dependent on exposure to wheat gluten and related proteins in rye and barley. Thus, these cereal proteins are necessary causal factors both to initiate and to maintain the disease process. On the other hand, epidemiological studies have shown that breastfeeding is a protective factor. Introduction of gluten-containing foods while the infant is still breast-feeding reduces the risk for celiac disease ${ }^{(17)}$. The age of the infant at introduction of gluten-containing foods does not seem to be an independent risk factor, but it is well established that introduction of gluten-containing foods in large amounts, as compared with small or medium amounts, increases the risk for celiac disease. Infectious episodes affecting the gut mucosal barrier could potentially contribute to the development of celiac disease in genetically susceptible individuals ${ }^{(17)}$.

Description of the pathophysiological mechanisms involved in celiac disease. HLA class II molecules are expressed on the surface of antigen-presenting cells where they can bind and subsequently present 'foreign' peptides encountered in the extracellular environment to populations of naïve T-helper lymphocytes that recognise the DQ2- or DQ8peptide complex. In the case of celiac disease, glutamine residues that remain in glutamine/proline-rich peptides following the intestinal digestion of 'gluten' are converted by tissue transglutaminase to negatively charged glutamic acid that binds to DQ2 or DQ8 molecules on antigen-presenting cells $^{(18)}$. The DQ-'gluten' peptide complexes activate DQ2 or DQ8 restricted T-helper cells, respectively, that proliferate and produce mainly Th1-type cytokines, particularly IFN- $\gamma$. 
Of note, such gluten-activated Th1 cells were isolated from the small intestinal mucosa of patients with celiac disease, but they are not found in the intestinal mucosa of individuals without celiac disease, who also have the relevant diseaseassociated DQ2 or DQ8 HLA class II molecules ${ }^{(18)}$. Secretion of Th1 cytokines such as IFN- $\gamma$ and TNF- $\alpha$ activates the release of enzymes such as matrix metalloproteinases that can damage the intestinal mucosa, with a loss of villus structure $^{(18)}$. These cytokines increase epithelial permeability and proinflammatory cytokine production, which in turn will increase the passage of gluten peptides and peptide binding to DQ2 and DQ8 molecules on antigen-presenting cells, leading to a chronic feedback of the inflammatory process as long as gluten peptides are present in the intestinal lumen.

A strict gluten-free diet for life is the only accepted treatment for celiac disease ${ }^{(19)}$. Successful outcomes are highly dependent on compliance to such a diet. Advanced celiac disease may not respond to a gluten-free diet, and these refractory cases are being treated with immunosuppressant drugs, including azathioprine and anti-TNF therapies with variable results (Fig. 2).

\section{Gut: inflammatory bowel diseases}

Definition and description of inflammatory bowel diseases. Ulcerative colitis (UC) and Crohn's disease (CD) are the two main forms of inflammatory bowel disease (IBD). UC and $\mathrm{CD}$ are both acute and chronic disabling inflammatory illnesses, whose aetiologies are unknown. Present opinion holds that IBD are multifactorial conditions, in which they involve both genetic and environmental components, with the final outcome being determined by an aberrant immune response to normal commensal microbiota in individuals who have a weakened epithelial barrier ${ }^{(20-22)}$. IBD is incurable, but typical maintenance treatments involve the use of immunosuppressant and anti-inflammatory drugs, antibiotics and surgery. An important factor differentiating CD from
$\mathrm{UC}$ is that it can affect any part of the gastrointestinal tract, not just the large bowel as in UC. Whereas CD is characterised by patchy, transmural inflammation with inflammatory processes occurring deep in the tissues, the inflammatory response in UC is primarily located in the colonic mucosa and submucosa resulting in severe tissue damage, ulceration and haemorrhage ${ }^{(23)}$. In both forms of IBD, large infiltrates of neutrophils can be seen in the inflamed tissues, together with the presence of microabscesses in the lamina propria. The main clinical feature of UC is bloody diarrhoea; other symptoms such as colicky abdominal pain and an urgency to defaecate may also be present, whereas in CD the symptoms can be more heterogenous and include diarrhoea, abdominal pain, weight loss and malaise ${ }^{(23)}$. Both CD and UC are associated with a 5-fold increased risk of developing colon cancer.

Epidemiology of inflammatory bowel diseases. Up to two million people are affected by IBD globally, with the disease being mainly associated with industrialised countries in the northern hemisphere. The incidence of $\mathrm{CD}$ is increasing in the UK and is about five to ten per 100000 per annum, with a prevalence of 50-100 per 100000 , although this is believed to be an underestimate ${ }^{(23)}$. Overall, the incidence and prevalence of UC is approximately twice that of CD. IBD usually starts early in life with the greatest onset being in young adults aged between 15 and 25 years, but it can affect people of any age, with $15 \%$ of people aged over 60 years at diagnosis.

Although a genetic component is known to be involved in IBD, with both $\mathrm{CD}$ and UC sharing some susceptibility genes, there is stronger evidence for a genetic link in $\mathrm{CD}^{(21,22)}$. In $\mathrm{CD}$, a $44 \%$ concordance exists between monozygotic twins, and a $4 \%$ concordance between dizygotic twins. Between 4.5 and $16.6 \%$ of $\mathrm{CD}$ patients have the disease already in the family, suggesting a polygenetic inheritance. A mutation in the NOD2/caspase recruitment domain-15 (called IBD-1) gene on chromosome 16 has been found in $30 \%$ of patients with $\mathrm{CD}^{(24)}$. NOD2 is a cytoplasmic receptor

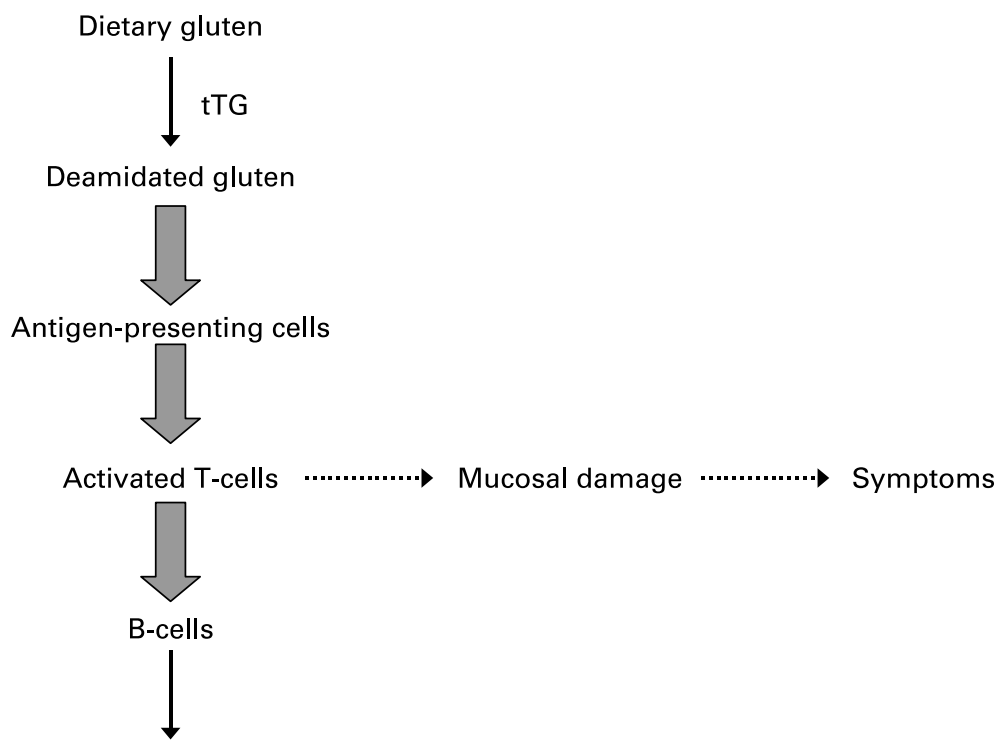

Anti-gluten and anti-tTG antibodies

Fig. 2. Generalised scheme for the development of celiac disease. 
for muramyl peptide found in bacterial cell walls, which may result in $\mathrm{CD}$ patients with this mutation having a reduced ability to clear invasive bacteria. A higher incidence of UC has been found in Jewish than in non-Jewish families, and Ashkenazi Jews born in the West have been found to have a higher incidence of $\mathrm{CD}$ than those born in Israel or nonAshkenazi Jews. Largely uncategorised environmental factors are thought to predispose towards IBD. However, the change to a more Western-style diet has been suggested as a link in IBD development. Smokers are more likely to develop CD and have more aggressive disease, with more severe relapses than non-smoking CD patients, although smoking has been shown to be protective in UC. Physiological stress, particularly long-term stress, may also be a precipitating factor in IBD. Stress can also bring about relapses in animal models of colitis and cotton-top tamarinds spontaneously develop colitis when kept in long-term captivity. Childhood illnesses and not being breast-fed are also thought to incur increased risk of developing IBD; however, appendectomy in early life can prevent the development of UC.

Description of the pathophysiological mechanisms involved in inflammatory bowel diseases. There is considerable evidence for microbial involvement in IBD. For example, while germ-free animals do not manifest an inflammatory response, knock-out or transgenic mice with genetic susceptibilities to IBD only acquire characteristic lesions when repopulated with normal commensal bacteria ${ }^{(25)}$. Human studies have shown that UC patients have increased levels of mucosal IgG against the normal colonic microflora, as well as increased antibody production against strictly anaerobic bacteria. UC also starts in the area of the gut with the highest concentration of micro-organisms. A number of different bacteria have been implicated in IBD in human subjects and animals, in various studies, but none have been definitively shown to be aetiologic agents in human subjects. The role of mucosal bacteria may be important due to their ability to interact more directly with the host immune system ${ }^{(26,27)}$. Nevertheless, $C D$ patients have been shown to have higher numbers of bacteroides and lower numbers of bifidobacteria in faecal $^{(28)}$ and mucosal samples, while higher numbers of peptostreptococci and lower numbers of bifidobacteria have been found on the mucosa in patients with $\mathrm{UC}^{(29)}$. Low numbers of bifidobacteria may be of significance in IBD because some species exhibit strong immunomodulatory properties ${ }^{(30)}$. This dysbiosis in mucosal bacterial populations in IBD, with the loss of beneficial commensal species, and perhaps a switch to a more pro-inflammatory phenotype, may explain why probiotic bacteria have been shown to be useful, in some cases, in the treatment of some forms of IBD, such as $\mathrm{UC}$ and pouchitis ${ }^{(27,29,31)}$.

In IBD, the interaction between the mucosal immune system and the commensal microflora in the gut is disturbed and dysregulation of the immune system occurs. The T-cell response profiles associated with $\mathrm{UC}$ and $\mathrm{CD}$ are different in that a Th1 pattern of cytokine formation develops in $\mathrm{CD}$ with increased production of TNF- $\alpha$, IFN- $\gamma$, IL-12, IL-6 and IL-1 $\beta$, whereas UC more resembles a modified Th2 profile, where cytokines including IL-5 and IL-10 are upregulated, although IL-4 is not ${ }^{(21)}$. In addition to this change in cytokine profile, intestinal $\mathrm{B}$ lymphocytes produce large amounts of IgG. TNF- $\alpha$ is expressed in the intestinal mucosa of patients with IBD and triggers inflammation via an NF- $\kappa \mathrm{B}$-dependent signalling cascade. Most of the pro-inflammatory responses in IBD are transcriptionally regulated by NF- $\mathrm{NB}$ and many of the cytokines involved act on signal transducers and activators of transcription (STAT) family ${ }^{(32)}$. IL-12 can act on CD4 + T-cells to induce differentiation into Th1 effector cells and activate the signalling proteins STAT-3 and STAT-4, which in turn leads to the production of IFN- $\gamma$. Animal studies have shown that STAT-4-deficient mice have impaired IFN- $\gamma$ production, and do not develop colitis, whereas mice overexpressing STAT-4 have diffuse infiltration of IFN- $\gamma$ and develop gut inflammation. STAT-3 signalling has been found in UC and CD where it has been shown to be confined to areas of active inflammation, infiltrating macrophages and T-cells. STAT-3 induces transcription of the pro-inflammatory cytokine IL-6, which can increase resistance of T-cells to apoptosis lengthening the chronicity of $\mathrm{CD}$, due to the accumulation of active T-cells. Increased numbers of blood mononuclear cells have also been found in IBD, which may lead to extraintestinal manifestations, while the production of free radicals from macrophages contributes to cellular damage. Other factors implicated in $\mathrm{CD}$ include generation of matrix metalloproteinases, such as collagenases and stromelysins, which can degrade extracellular matrices, cause ulceration and result in tissue destruction ${ }^{(22,33)}$. High levels of extracellular matrix metalloproteases, which can be upregulated by pro-inflammatory cytokines, have been shown in areas of tissue injury and foci of ulceration in CD patients. Group II phospholipase in serum and colonic mucosa has also been shown to be increased in patients with $\mathrm{CD}$, which can be inhibited by steroids and anti-inflammatory drugs. Corticosteroids are the main therapy used for treatment of active IBD, to downregulate the immune response, and allow the mucosa to heal. More recently, therapies involving the use of monoclonal antibodies such as Inflixamab against TNF- $\alpha$ have been found to be effective in inducing remission in CD.

\section{Lungs: airways and the allergic inflammation}

Normal functioning of the airways. The airways bring air into the alveoli of the lungs, where air and blood are separated only by the alveolar membrane that is about $0.001 \mathrm{~mm}$ thick. The turbulence of the air passing through the upper airways is such that most particles larger than $2 \mu \mathrm{m}$ in diameter are caught on the mucosal surface in the nasal cavity. This is beneficial with regard to starting an immune response against foreign material, because the back of the nasal cavity and the upper pharynx are rich in immune cells and lymphoid tissue. The trachea splits into two main bronchi that continue to branch out into bronchi and bronchioli, to end in the socalled alveolar sacs and alveoli. Alveoli have a good blood supply and are extremely thin-walled to allow gas exchange between blood and air. From the alveoli, the anatomical structure of the airways gradually changes towards the bronchi. The basic components are the epithelium that covers the inside of the airway tubes, and under the epithelium, the submucosa and then a layer with smooth muscle that can contract and thereby narrow the airways. In certain areas, the epithelial surface against the airway lumen has fine hair-like structures called cilia, which have a contractile 
apparatus and a synchronised beat so that mucus and particles will be moved up towards the larger airways and the larynx ('the mucociliary escalator').

Normal function of inflammatory responses to maintain homeostasis in the airways. The alveolar membrane $\left(140 \mathrm{~m}^{2}\right.$ surface area) is protected by three major, integrated defence systems along the airways. Firstly, the anatomy of the airways provides 'mechanical' defence, conditioning and cleaning the air as it passes through the nose. The mucociliary escalator helps remove particulate material. Secondly, the inflammatory response mediated by several cell types results in a rapid capacity to eliminate intruding microbes as well as foreign material and debris, but may become long-lasting if the material is not properly removed (in this case lung disease may result). Finally, the specific immune response is highly effective at eliminating microbes and other foreign material. The human lung is exposed daily to between 10000 and 20000 litres of ambient air containing large numbers of particles and gases that may cause oxidative stress and inflammation. Not surprisingly, the lungs have several systems to counter oxidative stress ${ }^{(34)}$.

Description of inflammatory airway and lung diseases: rhinoconjunctivitis, asthma and chronic obstructive pulmonary disease. The main diseases of the airways to be addressed are allergic rhinoconjunctivitis, allergic asthma and chronic obstructive pulmonary disease (COPD). These three are usually described as separate entities, but it is important to realise that there is a strong link between allergic rhinoconjunctivitis and asthma, and that asthma and COPD may be part of a continous spectrum of disease. At the one end of this spectrum would be allergic asthma with near-complete reversibility of bronchial obstruction and little inflammatory change and at the other end COPD with nearly no reversibility of airflow limitation, and pronounced inflammation and destruction of lung tissue. If asthma and COPD are considered as separate diseases, then many patients present components of both diseases to varying degrees. These components of airway disease include signs like variable airflow limitation, airway hyper-responsiveness, chronic airflow limitation and airway inflammation with different characteristics, as well as bronchiectasis and emphysema. Symptoms include chest tightness, wheezing, dyspnoea, cough and sputum and are all non-specific, since they can result from several disease components. Measures of lung function and inflammation are necessary to be certain as to which condition the symptoms are associated with ${ }^{(35)}$. Thus, many patients with COPD and asthma can be readily distinguished from one another, but many patients also have features of both disorders $^{(36)}$.

Allergic rhinoconjunctivitis is a fairly 'pure' allergic disease, in which the classical anti-allergen IgE-mediated release of histamine and other mediators from mast cells triggers the classical symptoms and signs of itching, sneezing, oedema, influx of eosinophils and watery mucous production. In contrast to allergic asthma, in rhinitis, there is no smooth muscle constriction narrowing the airways (narrowing is caused by swelling of the mucosa), and no significant tissue destruction. Subjects with allergic rhinoconjunctivitis are often found to have bronchial hyper-reactivity, and may later develop asthma.

Asthma is considered a chronic inflammatory disease of the lungs. Asthma is traditionally classified into allergic and non-allergic asthma. Allergic asthma is considered to be the most common form of asthma in children, while in adults asthma without known allergen triggers is more common. However, the distinction depends on the demonstration of triggering allergens, and is, therefore, somewhat unclear. It may be argued that asthma and allergy at least sometimes may be parallel but separate conditions (e.g. 'non-allergic' asthma in an individual that also has allergies). With both types of asthma, various 'non-specific' irritants may aggravate asthma and trigger an asthmatic attack, and the allergentriggered allergic inflammation can be looked upon as one of several such asthmagenic stimuli. Asthma has chest tightness, wheeze, cough and dyspnoea as prominent symptoms, and is functionally characterised as reversible bronchial obstruction, caused by contraction of the smooth muscle layer in the mucosa of the bronchi, by mucous production, mucosal oedema and mucosal inflammation. Airway hyperresponsiveness (oversensitivity and overreactivity to stimuli) is typically present in asthma, but is not an obligatory feature. Similarly, bronchial hyper-responsiveness is often found in individuals without manifest asthma. A prominent cell in the asthmatic inflammation is the eosinophil, together with lymphocytes. Granulocytes other than eosinophils may be present to varying degrees. The inflammation may lead to destruction and shedding of the epithelial cell layer. Over time, structural changes take place in asthma, so-called remodelling, inflammation will become permanent and more severe, and reversibility of the airways obstruction will become less complete. Chronic severe asthma may thus show features of COPD.

The definition of COPD rests much on functional criteria, with non-reversible or incompletely reversible airflow obstruction accompanied by shortness of breath, cough and sputum production, and intermittent exacerbations. However, some reversibility of airways obstruction may be present, and bronchodilators are the basic medication used in COPD. Underlying the symptoms are chronic lung inflammation and progressive, often pronounced tissue destruction. In addition to pulmonary manifestations, other organ systems may be affected in COPD. Systemic manifestations of COPD include weight loss, nutritional abnormalities and musculoskeletal dysfunction $^{(37-39)}$.

Epidemiology of allergic rhinoconjunctivitis, asthma and chronic obstructive pulmonary disease. Allergic rhinitis is triggered by allergens, but symptoms may be increased by air pollution. Allergic rhinitis may be constant (perennial) if the eliciting allergen is constantly present (e.g. mite allergens), seasonal if the allergen shows seasonal variation (e.g. in pollen allergy in the Nordic countries) or episodic (e.g. a person allergic to cats with low habitual exposure, who visits a home with cats). Allergic rhinitis starts in infancy and peaks in childhood and adolescence. Atopic individuals constitute the group at risk. In $80 \%$ of cases, the symptoms develop before the age of 20 years. Severity varies from trivial symptoms to periodically incapacitating disease. Allergic rhinitis may be associated with other upper-airway inflammatory conditions $^{(40,41)}$. A large international study showed considerable variation in prevalence between countries, from $0.8 \%$ to $14.9 \%$ in 6- to 7-year olds, and from 1.4 to $39.7 \%$ in 13 - to 14 -year olds ${ }^{(42)}$. There has been a general pattern of increased prevalence in the industrialised compared with the developing 
countries. Cross-sectional studies with similar methodology suggest a rising prevalence ${ }^{(43)}$.

Asthma severity varies from barely noticeable symptoms to lethal exacerbations. Prevalence of asthma can vary 3-fold in the same population depending on the diagnostic criteria used, and prevalence data should be interpreted cautiously ${ }^{(44)}$. Atopic individuals constitute the major risk group. However, as already mentioned, asthma is also frequently diagnosed in non-allergic individuals. Asthma is not only common in children but also develops in adult life. Diagnosis in small children is uncertain. For all age groups, incidence varies between countries from 2.7 to 4 per 1000 individuals per year. After 25 years of age, it has been estimated at 2.1 per 1000 individuals $^{(44)}$. Before puberty, asthma is more common in boys than girls, but after puberty this sex difference is reversed. Prevalence has been extensively studied in the last 30 years, and one major study is the International Study of Asthma and Allergies in Childhood ${ }^{(45,46)}$. The present study of nearly half a million children aged 13-14 years in 56 countries showed large variability between countries. The highest 12-month prevalence was found in the UK, Republic of Ireland, Australia and New Zealand (up to $36.8 \%$ ), and the lowest in Eastern European countries, Indonesia, China, India and Ethiopia (the lowest $1.6 \%$ ). A number of studies have found an increasing incidence and prevalence over the last decades ${ }^{(44,47)}$. However, some fairly recent studies have indicated that in some regions ${ }^{(48)}$, but not others ${ }^{(49)}$, the increase may have levelled out.

In 1990, COPD was the twelfth leading cause of morbidity and the sixth leading cause of death worldwide. Of all the major diseases, COPD is the one whose burden is rising the fastest, and it is projected to become the fifth leading cause of disability and the third leading cause of death by $2020^{(50)}$. COPD is strongly associated with cigarette smoking, but also occurs in non-smokers, and individuals vary greatly in their susceptibility to the effect of tobacco smoke. COPD is markedly underdiagnosed and frequently undermanaged, perhaps because it has been considered irreversible and that treatment has little to offer. However, COPD may be partially reversible and clinical responses to treatment do occur ${ }^{(51)}$.

Description of the pathophysiological mechanisms of lung disease, with an emphasis on inflammation

Environmental triggers. Asthma appears to be caused by environmental factors, such as allergens, irritants and infections, in genetically predisposed individuals. Once asthma has become manifest, essentially the same agents may trigger worsening of the disease and precipitate serious asthmatic attacks, as well as contribute to the development of chronic disease. A host of triggering as well as some protective factors are known, but the basic understanding of why and how asthma develops is still rudimentary. A major factor contributing to the development of allergy and asthma is the so-called Western lifestyle. This is illustrated by the 2- to 3-fold higher prevalence of asthma in former West Germany compared with East Germany, and Hong Kong compared with nearby cities in China ${ }^{(52,53)}$. Gradients in the prevalence of allergic disease corresponding to social gradients are also found within individual countries, and allergic diseases have been described as a price paid for wealth and a high standard of living.
Environmental and lifestyle factors have changed in a broad sense over the last decades in industrialised countries, but it is not known which specific conditions in the more affluent, industrialised or urbanised lifestyle drive the development of asthma and allergy. Also, asthma is a major health problem among the poor populations living in deprived inner city districts and the homeless in USA, with a very high prevalence of severe asthma, and prevalence is also high in some other underprivileged regions e.g. in South America. Asthma therefore does not, in all places, fit the 'hygiene hypothesis' model of more allergic disease among the 'clean and rich' and less among the poor (see later).

Allergen exposure will trigger asthma once the disease has become manifest, as will a wide array of non-specific irritants in the occupational setting, indoor environmental factors (e.g. second-hand tobacco smoke, strong odours and perfume) and outdoor air pollution factors like fine particulate matter, ozone and oxides of nitrogen. Also, physical exercise and psychological stimuli may precipitate asthma ${ }^{(44)}$.

For the causation of asthma, loss of protective environmental factors may be as important as asthma-provoking factors. The observation that the risk of asthma is reduced with increasing number of older siblings was explained by the Th1-Th2 pardigm of immune responses (see later) and the so-called hygiene hypothesis. In its original form, this hypothesis stated that younger siblings got more infections and got them earlier than the older siblings, and that the infections stimulated the development of Th1 immunity and counteracted the development of allergy-associated Th2 responses ${ }^{(54)}$. Although the focus has moved away from the hygiene hypothesis in the original form ${ }^{(55-57)}$, it is still considered that 'something with micro-organisms' is protective in relation to asthma and allergy ${ }^{(58)}$. Observations that more or less indirectly support this include the inverse relationship between family size and asthma ${ }^{(59)}$, the protective effect of early placement in day care settings ${ }^{(60)}$, the protective effect of exposure to farm animals ${ }^{(61)}$, the protective effect of endotoxin exposure (allergy more than asthma) ${ }^{(58)}$, the reduced prevalence of asthma among anthroposophic Steiner school attendants $^{(62)}$, the protective effect of extensive exposure to cats and $\operatorname{dogs}{ }^{(63)}$, the association between intestinal flora and atopic manifestations ${ }^{(64)}$ and the association between early use of antibiotics and increased incidence of allergy ${ }^{(65)}$. Other hypotheses of asthma causation include reduced intake of antioxidants from foods like fruit and vegetables ${ }^{(66,67)}$ and an altered balance of fatty acids in the diet ${ }^{(68)}$. Finally, obesity $^{(69)}$ and physical inactivity ${ }^{(70)}$ are associated with asthma and bronchial hyper-reactivity. Whatever the explanation, asthma must be said to represent 'an epidemic of dysregulated immunity, ${ }^{(71)}$.

For COPD, the major known environmental trigger is tobacco smoke. Also occupational chemicals and particulate matter may contribute to the development of the disease, as well as indoor and outdoor air pollution. Diet has been reported to be a modifying factor, with fruit and vegetable consumption having a protective effect ${ }^{(72)}$.

Perpetuating factors. In allergic diseases, like allergic rhinoconjunctivitis and allergic asthma, repeated and chronic allergen exposure will keep the disease active and worsening. Typically, general hypersensitivity and hyper-reactivity also to non-specific stimuli develop. These will contribute to 
perpetuating the disease, and viral infections and environmental pollutants (e.g. tobacco smoke and diesel exhaust particulates) will worsen the disease and may precipitate acute attacks of severe asthma. Largely, the same non-specific stimuli as seen in asthma, but in particular tobacco smoke, will contribute to perpetuating and accelerating COPD.

Mechanisms and mediators. Alveolar macrophages in the lumen of the airways are first-line defence cells, capable not only of engulfing foreign material to destroy it, but also of secreting several signal molecules (cytokines) that serve to attract and activate other cells to create inflammation. Macrophages can remove residual material after phagocytosis in two ways. They can go through the bronchiolar and alveolar walls to enter the lymphatic system carrying fluid and cells to the numerous lymph nodes located along the trachea and bronchi, or move out along the airways helped by the mucociliary escalator mentioned earlier, to be swallowed into the stomach or spat out. Particulate matter that is not dissolved may remain in the lymph nodes for a long time.

Epithelial cells are themselves active participants in immunoregulation and inflammation by secreting a number of inflammatory and immunoregulatory molecules. Furthermore, specialised cells (goblet cells) secrete protective mucus to cover the epithelial surface, and so-called type 2 cells secrete a family of molecules collectively referred to as surfactant. The surfactant has a number of beneficial functions, among others to regulate immune responses and inflammation $^{(73)}$.

In the submucosa, various cells of the specific and nonspecific immune systems can be found; among them, mast cells are important also in allergic reactions. The blood vessels bring various immune cells to the lung submucosa and epithelium. These include various subgroups of $\mathrm{T}$ and $\mathrm{B}$ lymphocytes and natural killer cells, monocytes, neutrophils, eosinophils and other cells. These various cells, in addition to performing defence tasks by cell-to-cell contact, carry with them or produce on-site two groups of molecules, signal molecules and defence molecules (with somewhat overlapping functions). Signal molecules more or less specifically regulate functions in other cells. For example, CD4 + T lymphocytes can secrete the cytokines IL-13, IL-4 and IL-9 to stimulate mucous production by epithelial cells, IL-13 and IL-9 to stimulate bronchial muscle contraction, IL-4 and IL-13 to stimulate antibody production by B lymphocytes, IL-3, IL-4, IL-5, IL-9, IL-13 and granulocyte-macrophage colony stimulating factor to attract and activate various other cells (sometimes in a very specific way), and IL-3, IL-4, IL-9 and IL-13 to stimulate the development of other cells. Accumulation and activation of cells is an important feature of the inflammatory response, and the cytokines mentioned will more or less contribute to such a response. Some other cytokines are particularly important for inflammation, like TNF- $\alpha$, IL-6, and IL-8 and IL-17, while IL-10 tends to downregulate inflammation as well as immune responses - an important function to protect against selfdestruction. Other defence molecules are designed to directly destroy the intruders: lysozyme; lactoferrin; complement; $\alpha-1$ antitrypsin; cationic proteins; lysophospholipases; catalases; non-specific esterases; others. The various granulocytes and the mast cells have in their cytoplasm granules containing preformed mediators that are released in the 'combat' situation.
At the heart of the allergic reaction is the interaction between $\operatorname{IgE}$ molecules bound to specific receptors on the membrane of mast cells and their corresponding allergens. When the IgE molecules are cross-linked by allergen, the mast cell is triggered to release the potent inflammatory mediators contained in its cytoplasmic granules, and the allergic inflammatory response develops. This response has two phases, an early virtually immediate reaction and a late response developing after some 6 to $8 \mathrm{~h}$. Mast cells are the key cells in the early response, while eosinophils are the predominant cell in the late response. Eosinophils are thought to play a central role in allergic rhinitis as well as asthma ${ }^{(41,74)}$. Asthma historically changed from being looked upon as a disease of the smooth musculature of the bronchi to being a Th2-dominated chronic inflammatory disease, with the eosinophil as the typical infiltrating cell. Other cells that regulate Th2 responses, asthma and allergy are NKT cells ${ }^{(75)}$, and the various types of regulatory T-cells (Treg cells) that have the capacity to inhibit the effector function of Th2 and Th1 cells $^{(76)}$. Increased levels of the Th2 cytokines IL-4, IL-5, IL-9 and IL-13 have been demonstrated in the asthmatic airway ${ }^{(77)}$. The Th2-driven inflammation has two arms, one via B-cells activated by IL-4 to produce $\operatorname{IgE}$, which triggers the mast cell-mediated allergic inflammation, and the other via IL-4-, but mainly via IL-13-mediated direct effects on epithelium and bronchial smooth muscle ${ }^{(78)}$. However, whereas the Th1/Th2 concept revolutionised immunological thinking when first proposed in 1987, it has been challenged over the past several years for several reasons ${ }^{(56,79)}$. Both Th1 and Th2 cells are pro-inflammatory, and Th1 cells do not always downregulate Th2 cells, but may instead exacerbate Th2mediated diseases ${ }^{(80-82)}$. It is of interest that the Th1 cytokine IFN- $\gamma$, now considered an aggravating factor in severe asthma, is also considered to be important in $\mathrm{COPD}^{(83)}$. Recently, TNF- $\alpha$ has also been reported to play an important role in severe asthma ${ }^{(84)}$. That Th1 cells play a role in asthma development is also illustrated by the finding that the Th1 transcription factor T-bet controls features of asthma. In the absence of T-bet (e.g. in T-bet-deficient mice), airway remodelling and airway hyper-reactivity develop spontaneously ${ }^{(85)}$. Additionally, it has been demonstrated that different asthmatic individuals display different 'individualised' cytokine patterns, caused probably both by different genetic factors and by differences in environmental exposure ${ }^{(82)}$. Neural mechanisms are central in the asthmatic inflammation ${ }^{(86,87)}$, but may also have a capacity to downregulate inflammation as has been shown in gastrointestinal inflammation ${ }^{(88)}$.

A number of genes have been implicated in asthma, e.g. ADAM $33^{(89)}$. It is estimated that more than a dozen polymorphic genes regulate features of asthma like the inflammatory response, IgE synthesis, cytokine and chemokine production, airway remodelling and airway function ${ }^{(90)}$.

On the molecular level, oxidative stress appears to play an important role in asthma ${ }^{(91,92)}$, and the endogenous antioxidant capacity of the lungs has been found to be defective in asthmatic patients ${ }^{(93)}$. Oxidative stress is caused by ROS. The inflammatory cells recruited into the asthmatic airways have a capacity to produce ROS as part of normal antimicrobial defence. Also, exogenous factors precipitating asthma, not only ozone and oxides of nitrogen, but also fine particulate matter, appear to cause oxidative stress $^{(94,95)}$. In animal 
models, factors that inhibit increased ROS generation have been found to reduce inflammation and hyper-reactivity, two important components of asthma ${ }^{(96)}$

While irreversible airflow limitation is a functional hallmark of COPD, the lesion is histopathologically characterised by inflammation and tissue destruction. Dominating inflammatory cells are neutrophils, macrophages and CD8 + -T lymphocytes and IFN- $\gamma$ is an important cytokine ${ }^{(83)}$. Oxidative stress appears to be important in the inflammation and tissue damage in COPD. Environmental agents linked to COPD, like tobacco smoke and particulate matter, induce oxidative stress and inflammation. Increased levels of markers of oxidative stress have been found in exhaled breath condensates of COPD patients $^{(97-99)}$, and in a mouse model of tobacco smokeinduced COPD tissue, damage due to oxidative stress was observed. Corticosteroid resistance in COPD has also been related to oxidative stress. An imbalance between protease and anti-protease enzymes is thought to be of importance for the development of the emphysema component of $\operatorname{COPD}^{(100)}$, and this is supported by findings in experimental models ${ }^{(101)}$.

\section{Joints: rheumatoid arthritis}

Normal function of inflammatory processes to maintain homeostasis. Controlled inflammation as a result of various triggers is likely to occur in normal joints. Possible triggers are local mechanical stress and transient infection. Local mechanical stress leads to induction of cellular stress proteins, which can be targeted by stress protein-specific regulatory T-cells ${ }^{(102)}$. Besides genetic factors and infection, there is some conflicting data on failing $\mathrm{T}$-cell regulation as a critical factor in the aetiology of rheumatoid arthritis (RA) ${ }^{(103)}$.

Definition and description of rheumatoid arthritis. RA is a common autoimmune disease characterised by chronic inflammation of the synovium of diarthrodial joints ${ }^{(104)}$. It can lead to long-term joint damage, resulting in chronic pain, loss of function and disability. Primarily, the small joints of the extremities are affected, but as the disease progresses joints become involved. The chronic inflammatory process changes the cellular composition (cellular infiltration) and the gene expression profile of the synovial membrane, resulting in hyperplasia of the synovial membrane, which causes structural damage in cartilage, bone and ligaments. Extra-articular disease affecting a variety of organs is a significant factor in morbidity and mortality of $\mathrm{RA}^{(105)}$. The severity of RA encompasses a wide spectrum, ranging from self-limiting disease to chronic progressive disease, causing varying degrees of joint destruction and extra-articular organ involvement.

Epidemiology of rheumatoid arthritis. RA occurs in $0.5-1.0 \%$ of the population worldwide ${ }^{(106)}$. RA is about two to three times more common in women than men. No increased incidence of RA has been noted over recent decades. In fact, decreases were documented, although this might have been artificially caused by a changed clinical classification of diseases previously characterised as $\mathrm{RA}^{(107)}$. Juvenile idiopathic arthritis (JIA) is one of the most common autoimmune diseases in childhood ${ }^{(108)}$. Although JIA has strong histopathological similarities to RA, it forms a separate clinical entity. Whereas untreated RA generally is progressive, JIA consists of different subtypes with striking differences in both severity and outcome. One subtype is persistent oligoarticular JIA, characterised by a remitting and often spontaneously selflimiting course. As a result of this, persistent oligoarticular JIA is unique among all autoimmune diseases. The selflimitation of an autoimmune process is often seen in experimental animal models but hardly ever in human subjects and seems to suggest the active involvement of immunoregulatory processes, such as regulatory T-cells. Indeed, the frequency of $\mathrm{CD} 4+\mathrm{CD} 25+$ regulatory $\mathrm{T}$-cells in the peripheral blood and in the synovial fluid of JIA patients has been found to correlate with the clinical course of the disease $^{(109,110)}$. Neither hygienic nor unhygienic living conditions are associated with the risk of developing juvenile $\mathrm{RA}^{(111)}$. Although the causes of RA remain elusive, the general consensus is that factors contributing to its occurrence and course (clinical heterogeneity) are probably both genetic and environmental. The main risk factors for the disease include genetic susceptibility, sex and age, smoking and infectious agents. In addition, hormonal, dietary, socio-economic and ethnic factors seem to contribute ${ }^{(112)}$. For RA, the main predisposing genetic factor is HLA-DR4. HLA-DR4 predisposes for a more severe progressive disease course. Besides HLA-DR4, other genetic factors have recently been discovered in RA, such as the genetic polymorphisms in the lymphoid protein tyrosine phosphatase (LYP), associated with both type 1 diabetes and $\mathrm{RA}^{(113)}$. As LYP is responsible for the tuning of T-cell activation, genetic variation of LYP will lead to variation in overall lymphocyte reactivity. The presence of anti-cyclic citrullinated peptides (CCP) antibodies (see later) seems to be an indicator for a higher grade of joint destruction and disease persistence. Smoking was found to be a risk factor for the development of anti-CCP antibodies. Anti-CCP positive RA may be a distinct disease entity that can react differently to treatment ${ }^{(114)}$.

Pathophysiological mechanisms of rheumatoid arthritis. RA involves many elements of the immune response. The synovium (or synovial membrane) is normally a relatively acellular structure consisting of one or two layers of synoviocytes. In RA, the synovium becomes hypertrophic and oedematous. Angioneogenesis, recruitment of inflammatory cells due to production of chemokines, local retention and cell proliferation contribute to the accumulation of cells in the inflamed synovium. Locally expressed degradative enzymes digest extracellular matrix and destroy articular structures $^{(115)}$. The synovial membrane that extends to the cartilage and bone is known as pannus. It actively invades and destroys the periarticular bone and cartilage at the margin between synovium and bone. T-cells are actively involved in the pathogenesis of RA. Activated T-cells that are abundantly present in the inflamed joints of RA patients can stimulate other cells (e.g. B-cells, macrophages and fibroblast-like synoviocytes) ${ }^{(116)}$. These T-cells are found to participate in the complex network of cell- and mediatordriven events leading to inflammation and joint destruction. B-cells play several critical roles in the pathogenesis of RA. They are the source of autoantibodies being produced in RA and contribute to immune complex formation (rheumatoid factors reactive with the constant region of their autologous IgG molecules) and complement activation in the joints ${ }^{(117)}$. Multiple other autoantibodies have been found in RA, with recent interest focused on those directed at $\mathrm{CCP}^{(118)}$ Antibodies that are directed to citrullinated protein ${ }^{(119)}$ 
detect $\mathrm{CCP}$ in many different proteins and are present in about $80 \%$ of the RA patients. Several lines of evidence suggest that citrullinated antigens have direct involvement in the rheumatoid disease process. Anti-CCP antibodies precede the clinical development of synovitis by many years. B-cells are also very efficient antigen-presenting cells, and can contribute to T-cell activation. The important role of B-cells in the disease aetiology is supported by the recent success of B-cell depletion therapy using rituximab ${ }^{(120)}$. The major effector cells in the pathogenesis of arthritis are synovial macrophages and fibroblasts. Activated macrophages are critical in RA, not only due to macrophage-derived cytokines (in particular TNF- $\alpha$ and IL-1) in the synovial compartments ${ }^{(121)}$, but also because of their localisation at strategic sites within the destructive pannus tissue. The variety and extent of macrophage-derived cytokines in RA and their widespread effect indicate that macrophages are local and systemic amplifiers of disease severity and perpetuation ${ }^{(122)}$. Among the many cell types present in the rheumatoid joint, the fibroblast-like synovial cell is prominent. It is now accepted that these cells are directly responsible for cartilage destruction, and also drive inflammation ${ }^{(123)}$. There is evidence for proliferation and expression of inflammatory cytokines and chemokines by fibroblast-like synovial cells in inflamed synovia. Various studies have shown the presence and activity of regulatory T-cells in RA, both in peripheral blood and in the synovial compartment during active disease ${ }^{(103,109)}$. Although in one of the RA studies anti-TNF interventions were shown to restore a compromised activity of regulatory $\mathrm{T}$-cells ${ }^{(124)}$, it seems that impaired $\mathrm{T}$-cell regulation is a more prominent feature of multiple sclerosis and type 1 diabetes than RA.

\section{Skin: atopic eczema and psoriasis}

Atopic eczema (also called atopic dermatitis) and psoriasis are among the commonest inflammatory skin diseases. Both involve interactions between the immune system and the skin. Although broad acting immunosuppressive therapies are effective against both, very different pathogenetic mechanisms are involved.

Atopic eczema. Fifteen per cent of children will have eczema at some time during the first 12 years of their life ${ }^{(125)}$. The clinical manifestations usually appear within the first year of life and often within a few weeks of birth. The rash of eczema is characteristically distributed (flexures) over ill-defined areas of intensely itchy redness containing an infiltrate of $\mathrm{T}$ lymphocytes and eosinophils. There is a clear genetic predisposition but expression of the phenotype is determined by environmental factors (among those suggested are lack of bacterial exposure providing an immune stimulus, intra-uterine exposure to allergens, poor fetal nutritional status). A major component in the pathogenesis of atopic eczema is the involvement of the immune system through a combination of immediate type (IgEmediated) and T-cell-mediated immune hypersensitivities to environmental aeroallergens and to food allergens. Although the different types of hypersensitivity are demonstrable by prick test, intradermal injection or epicutaneous patch test challenge with a range of allergens, proving that the causal relevance of specific allergies is difficult and unreliable. The main effective therapies for atopic dermatis include topical steroids and systemic immuno-suppressives.
A fundamental component of the atopic state appears to be a dysregulation of the immune system such that $\mathrm{T}$ lymphocytes responding to atopic allergens differentiate towards the Th2 phenotype ${ }^{(126,127)}$. This may represent a failure of maturation of the fetal immune system, which is maintained during pregnancy with a bias towards Th2 responses ${ }^{(128)}$. Signals from DC are thought to determine the differentiation pathway of Th cells. Microbial components including lipopolysaccharides signalling through innate receptors such as toll-like receptors are critical in this, as reflected by the hygiene hypothesis ${ }^{(54,129-131)}$. This proposes that early life exposure to microbes accelerates the maturation of the immune system away from the fetal Th2 bias and towards the adult Th1-biased differentiation pathway. Monocytes from atopic eczema sufferers release increased quantities of PGE2, which can drive T-cell differentiation towards the Th2 phenotype ${ }^{(132,133)}$.

Psoriasis. Two to three per cent of the population suffer from psoriasis; $10-15 \%$ of these have it severely and require management by dermatologists. Fifty per cent of these will have significant joint symptoms and psoriatic arthritis. There is a genetic susceptibility and a number of genetic loci have been identified that may contribute to this susceptibility. There are associations with hyperlipidaemia and $\mathrm{CHD}^{(134,135)}$ as well as $\mathrm{CD}^{(136)}$. Various factors are involved in the expression of the psoriatic phenotype: streptococcal infections; psychological stress; physical trauma to the skin. The rash of psoriasis is characterised by plaques of red scaly skin characteristically distributed over bony prominences. The pathophysiology involves an interaction between the immune system and the skin. There is an infiltrate of T lymphocytes (both CD4 + and CD8 + ) into the dermis, formation of clusters of neutrophils in the epidermis, involvement of two or three layers of the epidermis in proliferation and a greatly accelerated but incomplete differentiation. There are also proliferation and altered structure of the dermal capillaries, which become tortuous and dilated. Activation of the innate immune system by streptococcal products and, most likely, as yet unidentified factors, induces release of cytokines including IFN- $\alpha$ and $-\gamma$. The cellular source is unclear but could be plasmacytoid DC or other DC. This activates keratinocytes to proliferate and produce angiogenic factors that induce proliferation of dermal microvessels. The fundamental abnormality has not been identified, but the central feature is a failure of mechanisms of resolution of inflammation. The main treatment modalities include agents that modulate both keratinocyte proliferation and activation of T-cells. These include topical agents and systemic drugs. While many therapies have diverse effects on immune cells, cytokine production and/or cellular proliferation, it is unclear what the critical therapeutic target is. Retinoids, vitamin D analogues and glucocorticoids all have significant therapeutic efficacy and have in common action via the superfamily of ligand-activated nuclear transcription factors. The possibility is that nutritional intervention is suggested by the evidence of the involvement of eicosanoid mediators in psoriasis. Thus, there are reports that inhibition of leukotriene B4 (LTB4) with benoxaprofen was effective against psoriasis ${ }^{(127,138)}$.

\section{The vascular wall: CVD}

Medium-sized arteries, such as the coronary vessels, consist of three compartments termed the intima, media and adventitia, 
respectively. The intima is lined by a single continuous layer of endothelial cells. The endothelium forms the interface between the constituents of blood and the artery wall. Collectively, the cells of the endothelium have a mass of more than a kilogram and a surface area equivalent to that of a football pitch. Endothelial cells differ in their properties at different anatomical sites in the vascular tree. Such differences include variations in permeability and cytokine expression. Endothelial heterogeneity may be partially responsible for the characteristic regional distribution of atherosclerotic lesions described in human post-mortem studies. The endothelium appears to play a critical role in the regulation of vascular tone, and inhibiting leucocyte adhesion and platelet aggregation, through its release of mediators such as $\mathrm{NO}$ and prostacyclin $^{(139)}$. NO is derived from L-arginine through the action of the constitutive form of the enzyme, endothelial NO synthase. It inhibits platelet aggregation and adhesion, modulates smooth muscle cell proliferation, attenuates the generation of endothelin and reduces leucocyte adhesion to the endothelium. The intima and media are separated from each other by a thin layer of elastic tissue called the internal elastic laminar. The media is composed of spirally arranged smooth muscle cells responsible for the generation of tone within the artery and determining the luminal diameter.

Atherogenesis. Atherosclerosis, or 'hardening of arteries' is the major cause of CVD. It is most often due to the formation of atheroma. Endothelial dysfunction is thought to presage atherosclerosis, and is characterised by altered endothelial function, enhanced adhesion molecule expression, increased leucocyte adhesion and impaired endotheliumdependent vasodilator responses ${ }^{(140-143)}$. Although the endothelium appears to continue to elaborate NO, its biological activity appears to be compromised in the early phases of atherogenesis $^{(144)}$. The latter is likely to be due in part to the interaction of NO with other molecular species such as the superoxide radical. These interactions neutralise the protective effects of NO and generate products, such as peroxynitrite that may be cytotoxic and pro-inflammatory. These changes in the properties of the endothelium have given rise to the concept of endothelial dysfunction. Endothelial dysfunction is associated with enhanced thrombosis and impaired fibrinolysis, and a number of risk factors appear to contribute to endothelial injury, including smoking, hypertension and hyperlipidaemia. The emergence of the 'lipid oxidation' hypothesis ${ }^{(145)}$ provided yet another mechanism of endothelial injury, and an explanation for the formation of lipid-laden, macrophage-derived foam cells. Uptake of cholesterol by cells is normally mediated by the LDL receptor, and in the presence of high cellular levels of cholesterol, the LDL receptor is downregulated, limiting cholesterol accumulation. However, the uptake of oxidatively modified LDL is mediated by 'scavenger receptors' and this mechanism is not regulated by cellular cholesterol content. The putative protective effect of antioxidants, such as vitamin $\mathrm{E}$, that inhibit LDL oxidation is explained by this hypothesis, although it is important to note that these effects remain contentious ${ }^{(146,147)}$.

Leucocytes become attached to the dysfunctional endothelium and subsequently accumulate within the subendothelial space ${ }^{(148,149)}$. Macrophages are converted to lipid-laden foam cells within the artery wall, giving rise to a lesion termed the fatty streak. The initial adhesion event is mediated by pairs of adhesion molecules. The endothelial adhesion molecules have been shown to be upregulated early in atherogenesis.

The progression of the atherosclerotic plaque is complex process, and in its early stages of development may be reversed ${ }^{(150,151)}$. The conversion of the fatty streak into a fibrous plaque necessitates the recruitment and proliferation of vascular smooth muscle cells ${ }^{(152)}$. This process is driven by the synergistic interplay of several growth factors.

Fatal myocardial infarction is usually associated with thrombi and plaque fissuring. Unstable plaques are thought to be particularly prone to fissuring, and these are characterised by a large lipid pool, thin fibrous cap and large numbers of inflammatory cells ${ }^{(153)}$. Activated macrophages within the plaque are a rich source of matrix metalloproteinases that have the ability to degrade extracellular matrix ${ }^{(154)}$. They therefore have the potential to destabilise the plaque, leading to localised regions of de-endothelialisation that may subsequently lead to focal thrombosis and plaque rupture. In the later stages of its evolution, a plaque may be stabilised. Although such lesions may be sufficiently prominent to cause luminal narrowing, and hence angina, or claudication, they are less likely to rupture, and are therefore considered relatively safe.

The role of chronic inflammation in atherogenesis. Atherosclerosis bears many hallmarks of a chronic inflammatory disease, and every stage of its evolution is characterised by monocyte/macrophage and T-lymphocyte infiltration ${ }^{(155)}$. The possible stimuli to this inflammatory process include oxidised LDL, homocysteine, free radicals generated from cigarette smoking, and infectious micro-organisms, such as Chlamydia pneumoniae. The T-cell infiltrates are predominantly CD4 + cells and clones derived from human lesions have been found to react to antigens derived from oxidised LDL, heat-shock proteins and micro-organisms ${ }^{(156)}$. The cytokine milieu within atherosclerotic lesions is thought to promote a Th1-dominated response associated with macrophage activation and the elaboration of IFN- $\gamma$ and IL-1 $\beta^{(156)}$. Hence, if the original insult is not adequately neutralised, inflammation may persist, causing the local and systemic release of growth factors and cytokines. These can, in turn, lead to intimal thickening by stimulating smooth muscle cell migration, proliferation and extracellular matrix elaboration.

Inflammatory biomarkers and atherosclerosis. There has been an increasing interest in the use of inflammatory markers to estimate the risks of acute events in patients with established coronary disease. In part, the predictive value of these markers may be related to their ability to identify patients with vulnerable plaques, which are rich in activated leucocytes. CRP, IL-6 and circulating leucocyte count are elevated in patients with myocardial infarction ${ }^{(157)}$. There have also been reports of a positive relationship between serum CRP and subclinical atherosclerosis ${ }^{(158)}$, although analysis may be confounded because risk factors such as smoking habit, indices of adiposity, blood pressure, TAG and HDL are also associated with CRP concentrations. Adipose tissue may be an additional source of inflammatory cytokines that could stimulate CRP production $^{(159)}$. Other inflammatory conditions, such as RA and systemic lupus erythematosus, are associated with impaired endothelial function and increased coronary risk ${ }^{(160,161)}$.

Epidemiology of CVD. The Framingham Heart and Seven Countries Studies were key to identifying cigarette smoking, 
high serum cholesterol, physical inactivity, high blood pressure, the menopause and psychological factors as contributors to coronary disease. The importance of multiple, low-level risk factors has become clearly evident ${ }^{(162)}$. Death rates from CVD have been falling in many developed countries, including most countries of Western Europe, since the early $1970 \mathrm{~s}^{(163-166)}$ and much of this reduction appears to be due to successful implementation of primary intervention due to dietary change, statin treatment, antihypertensive treatment and fall in smoking habit. The rate of reduction in CVD mortality differs among countries. For example, the fall in the UK is slower than that in Australia, Canada and Sweden. However, death rates from CVD have risen rapidly in many Eastern European countries. Poland is an exception to this because rates have fallen in association with a fall in the prevalence of smoking, reduced saturated fat and increased PUFA and fruit consumption ${ }^{(167)}$. Besides national differences, there are very marked regional differences within countries ${ }^{(166,168)}$, which may reflect socio-economic differences. The incidence of CHD varies with race and ethnicity ${ }^{(169)}$. In the USA, black men and women have a higher risk of CHD than white men and women, respectively, while Hispanic groups have lower CHD rates. South Asians living in the UK have approximately $50 \%$ higher rates of premature CHD death, and the difference is widening as rates do not appear to be falling as fast among this group as for the UK as a whole. This may relate to a higher prevalence of diabetes in this group.

Interventions. The landmark statin trials have demonstrated that cholesterol lowering reduces cardiovascular mortality and morbidity ${ }^{(170)}$. The early benefits observed in some of the primary prevention trials have suggested that statins may be exerting effects other than those attributable to cholesterol lowering alone, so-called 'pleiotropic effects' including effects on inflammation, although this remains a matter of considerable debate. The role of low-dose aspirin treatment in the prevention of coronary disease is also well supported by trial data ${ }^{(171)}$. However, the mechanism for the protective effect may relate to the anti-platelet effects of aspirin, rather than an effect on inflammation. Although trials of antibiotic treatment have generally not shown a benefit in preventing coronary disease ${ }^{(172)}$, it has been argued that diet regimens used to date may not eliminate the carriage of organisms by peripheral monocytes. There have been few studies describing the effects of dietary constituents on inflammation in healthy subjects or patients with CHD. Fish oil supplements, providing very long-chain $n$-3 PUFA (VLC $n-3$ PUFA) alter the composition of inflammatory cells resulting in decreased production of inflammatory eicosanoids and cytokines ${ }^{(173)}$, alter cellular composition of human plaques, making them appear more stable $e^{(174)}$, and reduce mortality from sudden death in secondary prevention ${ }^{(175,176)}$. Meta-analyses support lowered cardiovascular mortality with increased VLC $n-3$ PUFA intake ${ }^{(177,178)}$

\section{Adipose tissue: obesity}

Adipose-derived mediators. The prevalence of obesity (defined as a BMI $>30 \mathrm{~kg} / \mathrm{m}^{2}$ ) is rising throughout the world, and obesity is occurring at a younger age. In the US and most Western countries, obesity has an incidence rate above $20 \%$. This is a major public health issue because of the direct effect of obesity on the risk of developing type 2 diabetes, CVD, hypertension, stroke, abnormal blood lipids, arthritis, asthma and cancer ${ }^{(179)}$. Until very recently, the role of adipose tissue has been thought to be passive and adipocytes were considered as little more than fat stores. However, it is now clear that this is far from the case and that adipose tissue is an important endocrine organ ${ }^{(180)}$. There is increased understanding that, depending on the nutritional state, the profile of hormones released from adipocytes can change from being beneficial to being detrimental ${ }^{(181)}$. The adipocyte plays a major role in the inflammatory response and releases a cocktail of inflammatory mediators and signalling molecules ${ }^{(182,183)}$.

Four findings ignited the interest in the adipocyte as an endocrine organ. Firstly, the discovery of leptin in 1995 changed the way the adipocyte was viewed; the discovery that leptin has a role in signalling the status of adipose tissue to the brain was the first description of a feedback loop involved in body weight control ${ }^{(182)}$. Although its role in human subjects is more complicated, the importance of leptin as a hormone involved in energy balance has been exemplified by the case studies of families with leptin deficiency; the children are obese and lose weight with leptin therapy ${ }^{(183)}$. As human subjects gain weight, they seem to develop leptin resistance. This may be mediated by suppressor of cytokine signalling, which increases in the hypothalamus in tandem with leptin. This blocks the central effect of leptin ${ }^{(184)}$. Leptin has important effects on peripheral metabolism, which have been demonstrated through lipodystrophy models. Leptin has been shown to have a role to play in insulin sensitivity and TAG clearance from the circulation. This observation in evolutionary terms may have allowed weight gain in times of plenty without gross metabolic change.

Secondly, the discovery that adipocytes release TNF- $\alpha$ also changed the view of adipocytes ${ }^{(185)}$. In fact, mRNA expression studies show that adipocytes can synthesise TNF- $\alpha$ and several other cytokines, notably IL-1 $\beta$ and IL-6 among an ever-increasing list. Circulating TNF- $\alpha$ and IL- 6 concentrations are mildly elevated in obesity. Most studies suggest increased TNF- $\alpha$ mRNA expression or secretion in vitro in adipose tissue from obese subjects. The factors regulating cytokine release within adipose tissue appear to include not only typical inflammatory stimuli such as lipopolysaccaride, but also the size of the fat cells per se and catecholamines. The effects of cytokines within adipose tissue include some actions that might be characterised as metabolic. TNF- $\alpha$ and IL-6 inhibit lipoprotein lipase, and TNF- $\alpha$ additionally stimulates hormone-sensitive lipase. TNF- $\alpha$ also downregulates insulin-stimulated glucose uptake via effects on GLUT-4, insulin receptor autophosphorylation and insulin receptor substrate-1. All these effects will oppose lipid accumulation within adipocyte ${ }^{(186)}$. Other effects appear more trophic, and include the induction of apoptosis, regulation of cell size and induction of de-differentiation (the latter involving reduced PPAR- $\gamma$ ). Cytokines are important stimulators and repressors of other cytokines. In addition, cytokines appear to modulate other regulatory systems. Examples of the latter include effects on leptin secretion (probably stimulation followed by inhibition) and reduction in $\beta 3$-adrenoceptor expression. There seems to be no clear agreement as to which cytokines derived from adipose tissue act as remote 
regulators, i.e. hormones. Leptin, which is structurally a cytokine, is also a hormone. IL-6 appears to be released systemically by adipose tissue, but TNF- $\alpha$ is probably not. Both leptin and IL- 6 appear to act on the hypothalamus, IL- 6 acts on the liver, while leptin may have actions on the pancreas ${ }^{(186)}$.

Thirdly, the discovery of adiponectin, a protein which is specifically and very highly expressed in adipose tissue ${ }^{(187)}$. This hormone enhances insulin sensitivity in muscle and liver and increases fatty acid oxidation in several tissues, including muscle fibres. It also decreases serum fatty acid, glucose and TAG concentrations: if normal, lean mice are given injections of adiponectin in conjunction with a meal high in fat and sugar, the normal postprandial increases in plasma glucose and TAG concentrations are smaller as the result of an increased rate of clearance from the blood rather than a reduced rate of absorption from the gut. By contrast, if insulin-resistant mice are treated with physiologic concentrations of adiponectin, glucose tolerance is improved and insulin resistance is reduced. In human subjects, plasma adiponectin concentrations fall with increasing obesity and this effect is greater in men than women. Reduced adiponectin concentrations correlate with insulin resistance and hyperinsulinaemia. In addition, several polymorphisms of the adiponectin gene (APM1, mapped to chromosome 3q27) have been identified that are associated with reduced plasma adiponectin concentration and that increase the risk of type 2 diabetes, insulin resistance or the metabolic syndrome. Interestingly, adiponectin appears to be implicated in the development of atherosclerosis. Adiponectin concentrations are reduced in patients with $\mathrm{CHD}$, and adiponectin inhibits TNF- $\alpha$-induced expression of adhesion molecules and the transformation of macrophages to foam cells, both of which are key components of atherogenesis. In mice deficient in apo E (and thus susceptible to atherosclerosis), treatment with human adiponectin inhibits lesion formation in the aortic sinus by $30 \%$ compared with that in untreated control animals $^{(181,184)}$. The central importance of adiponectin is exemplified by the observation that it inhibits NF- $\kappa \mathrm{B}$ and reduces the proinflammatory pathway.

Fourth is the observation that adipose tissue is not homogeneous. Macrophage numbers in adipose tissue increase with obesity, where they apparently function to scavenge moribund adipocytes, which increase dramatically with obesity. Macrophages are responsible for most of the cytokine production in adipose tissue, especially in obesity. In fact, adipose tissue macrophages are responsible for perhaps as much as $50 \%$ of adipose tissue $\mathrm{TNF}-\alpha$ production ${ }^{(184)}$. In obesity, adipose tissue contains an increased number of resident macrophages so that, in some circumstances, macrophages can constitute up to $40 \%$ of the cell population within an adipose tissue depot.

An integrated view of inflammation and obesity. The issues surrounding weight gain and inflammation cannot be seen in isolation, but need to be viewed alongside theories about variation in adipocyte differentiation, appetite regulation and control of appetite. Recent evidence has suggested that the formation of hypertrophic fat cells in environments of energy excess may be due to a genetically determined differentiation limit of stem cells to adipocytes or metabolic feedback controlling differentiation or more likely a mixture of the two. If there are limited numbers of adipocytes in an environment of energy excess, this will cause excessive lipid filling in the adipocyte and lead to hypertrophic adipocytes that are proinflammatory. The key issue at the beginning of the inflammatory process seems to be an excess energy intake. Many of the processes that follow excess energy intake may acutely be an advantage, but an environment of chronic excess energy intake and decreased energy output will work against human health. As the adipocyte stores TAG, there seems to be an increase in cytokine release. Of particular importance is the suppression of adiponectin and the increase in TNF- $\alpha$. It is possible that $\mathrm{TNF}-\alpha$ can decrease proliferation and differentiation of adipocytes. The control mechanisms for adipocyte proliferation and differentiation are complex, but examination of the transcriptional and extracellular signals necessary for stem cell differentiation into a preadipocyte, and from the preadipocyte into a mature fat cell, is being elucidated. Regulation of adipocyte proliferation and differentiation involves growth arrest and the coordinate activation/inactivation of nuclear transcription factors that regulate the expression of genes necessary for lipid storage and insulin sensitivity. ADD/SREB-1, C/EBP-a, $-b$ and $-d$ and PPAR $-\gamma$ are a few of the transcription factors known to be involved. These transcription factors are regulated in response to extracellular signals, such as PG, cytokines, and hormones including corticosteroids and insulin ${ }^{(188)}$. Defects in any one of these steps are potentially important in the failure of proliferation or differentiation of adipocytes. This will limit the number of adipocytes coping with the excess energy intake. This will have several effects.

(i) Effects on recruitement of monocytes into adipose tissue. As the lipid content of adipose tissue increases, the adipocytes increase leptin output and this has the effect of increasing expression of adhesion molecules such as ICAM-1 and platelet-endothelial cell adhesion molecule-1 in endothelial cells inducing adhesion and transmigration of monocyte/macrophages. The transmigration of macrophages is further enhanced by monocyte chemoattractant protein-1. The result is a tissue that produces the low-grade systemic inflammation seen in obesity ${ }^{(189)}$.

(ii) Effects on apoptosis and TNF- $\alpha$. In mature white adipocytes, TNF- $\alpha$ is reported to regulate cell size, the suggestion being that as the cells get bigger they produce more TNF- $\alpha$, which in turn initiates changes to limit adipocyte size or to induce apoptosis. This is possibly coupled with increasing local hypoxia. The net result is recruitment of greater numbers of macrophages that surround the apoptotic cell ${ }^{(186,189)}$.

(iii) Autocrine effects on adipokines. The increase in TNF- $\alpha$ has been shown to have several autocrine effects including reduced expression of the GLUT4 glucose transporter, increased hormone-sensitive lipase activity leading to increased lipolysis, decreased lipoprotein lipase activity, reduced insulin signalling and decreased C/EBP and PPAR- $\gamma$ activity, so creating an insulin insensitive adipocyte with a high output of NEFA ${ }^{(186)}$.

(iv) Effects on peripheral tissue. From the earlier adipocyte hypertrophy, increased insulin resistance and increased fatty acid output result and excess dietary fat may be shunted to the liver, skeletal muscle and the $\beta$-cell. Importantly, if fat oxidation cannot be increased to compensate for the increased influx of lipid within 
these tissues, then intracellular accumulation of lipids will occur. The oxidation of fat is mediated by activating AMP-activated protein kinase (AMPK). It is known that adiponectin activates AMPK as does leptin. So, in normal physiology, leptin and adiponectin signal transduction involves the phosphorylation of STAT pathways, but how this translates into the observed changes in lipid metabolism is not clear. One likely mechanism is via increased phosphorylation activation of AMPK, which phosphorylates acetyl CoA carboxylase and malonyl CoA decarboxylase. Phosphorylation inactivates carboxylase, but activates malonyl CoA decarboxylase. Because carboxylase catalyses malonyl $\mathrm{CoA}$ formation, and malonyl $\mathrm{CoA}$ decarboxylase catalyses its decarboxylation, the net effect of AMPK activation on these target enzymes is to lower malonyl CoA concentration. Malonyl CoA is the first committed step in lipogenesis and a powerful inhibitor of carnitine palmityl transferase-1-mediated fatty acid oxidation. The combination of an increase in fatty acid oxidation and a decrease in fatty acid synthesis could account for the reduction in the lipid content of a cell. At present, however, we do not understand how leptin activates AMPK. In the inflammatory state there is low adiponectin and leptin resistance and therefore low activity of AMPK, and reduced oxidation of fat and the storage of TAG in cells. It is likely that it is products generated from TAG such as unoxidised palmitoyl-CoA that lead to insulin resistance and cell apotosis. Also diacylglycerol has been shown to have effects on protein kinase $\mathrm{C}$; this can in turn affect the insulin signal by causing serine phosphorylation rather than tyrosine phosphorylation on IRS-1 so reducing the insulin signal. This theory is supported by the recent observation that there is a direct relationship between hepatocyte and myocyte lipid content and insulin sensitivity. Interestingly, IL-6 can also affect steroid hormone conversion, changing the balance of sex hormones, which is well known to regulate adipose tissue distribution ${ }^{(190-192)}$.

The release of other cytokines (e.g. plasminogen activation inhibitor 1 (PAI-1)) from the adipocyte can have dramatic effects on risk of disease. There is evidence for an association between increased PAI-1 levels and myocardial infarction. Interestingly, PAI-1 has been shown to predict myocardial events in univariate analysis, but the predictive power was not affected by adjustement for inflammatory parameters and disappeared after adjustment for BMI, TAG and HDL cholesterol. This suggests that the metabolic syndrome is a precursor to high plasma PAI-1 levels in patients prone to atherosclerosis ${ }^{(193)}$.

Effect of weight loss on inflammatory markers. The induction of a negative energy balance has profound effects on inflammatory markers in obesity: weight loss with diet and exercise has been shown to decrease circulating IL-6 and TNF- $\alpha$ concentrations in obese subjects ${ }^{(194,195)}$. Serum CRP concentration fell 4 months after bariatric surgery but remained significantly elevated relative to non-obese controls $^{(196)}$. Very recently, there has been a description of a fall in proinflammatory cytokines and macrophage numbers in white adipose tissue following weight loss ${ }^{(195)}$. A recent study on obese children has demonstrated falls in plasma TNF- $\alpha$ and CRP concentrations with weight loss ${ }^{(197)}$. Interestingly, liposuction has very little effect on inflammatory markers ${ }^{(198)}$. The success of lifestyle intervention has been demonstrated with the American and Finnish Diabetes Prevention trials, which both show long-term benefit in decreasing the risk of type 2 diabetes $^{(199,200)}$.

\section{Common features of inflammatory processes that may be suitable targets for nutritional intervention}

Although inflammation-induced tissue damage occurs in an organ-specific manner (gut, lungs, joints, skin, blood vessel wall, adipose tissue) in different diseases or conditions, there is some commonality among the responses seen in the different organs (summarised in Table 1). In general, the inflammatory response observed is normal but it occurs in the wrong context, which relates to inappropriate barrier function (epithelial or endothelial), inappropriate triggering (i.e. a response to a normally benign stimulus equivalent to a loss of tolerance), lack of downregulation to control the response and tissue destruction with a loss of function. In some cases, the inflammation is the result of tissue damage caused by endogenous molecules such as necrotic cell debris or oxidised LDL. In other cases, it is the result of exogenous triggers such as allergens or microbes. The involvement of different triggers is also reflected in the distinct associations with polymorphisms in receptors involved in 'pattern' recognition such as NOD2 in CD or with other molecules involved in specific adaptive immune responses such as HLA-DR subtypes in UC and RA (Table 1). However, although trigger, localisation and resulting clinical symptoms are different, many of the processes, cells and molecules involved in the actual inflammatory response are remarkably similar (Table 1). Most, if not all, of the chronic inflammatory diseases considered here are characterised by overproduction of cytokines (TNF- $\alpha$, IL-1 $\beta$, IL-6, IFN- $\gamma$ ), chemokines (IL-8, MCP-1), eicosanoids (PGE2, four-series LTs) and matrix metalloproteinases. Elevated levels of these mediators act to amplify the inflammatory process (e.g. by attracting further inflammatory cells to the site) and contribute to tissue destruction and to the clinical symptoms observed. Many of these mediators are positively regulated through NF- $\mathrm{B}$ (Fig. 3) and some are negatively regulated through PPAR and liver $\mathrm{X}$ receptors. Entry of inflammatory cells to sites of inflammatory activity is facilitated by upregulation of adhesion molecules on the endothelium, a process that is promoted by inflammatory cytokines and by a range of inflammatory triggers, frequently acting through $\mathrm{NF}-\kappa \mathrm{B}$. The continuous process of tissue injury, healing and repair, due to the release of cytokines, chemokines and growth factors by infiltrating inflammatory cells as well as resident tissue cells, results in tissue remodelling. Local structural cells, such as fibroblasts, epithelial cells and smooth muscle cells can play a role in amplification of the inflammatory response and to a large extent determine the clinical appearance of the disease.

It is noteworthy that in many cases, at some point, the tissue damage appears to result in a loss of barrier function and exposure of hitherto sequestered antigens of either the host or of its microbiota. This can result in a loss of tolerance and sustained specific responses to such antigens. This can 
Table 1. Summary of the disease states discussed and an overview of their key inflammatory components

\begin{tabular}{|c|c|c|c|c|c|c|c|c|c|c|}
\hline & Coeliac disease & $\begin{array}{l}\text { Crohn's } \\
\text { disease }\end{array}$ & Ulcerative colitis & COPD & Asthma & $\begin{array}{l}\text { Atopic } \\
\text { dermatitis } \\
\text { (atopic } \\
\text { eczema) }\end{array}$ & Psoriasis & $\begin{array}{l}\text { Rheumatoid } \\
\text { arthritis }\end{array}$ & Atherosclerosis & Obesity \\
\hline $\begin{array}{l}\text { Organ } \\
\text { affected }\end{array}$ & Small intestine & $\begin{array}{l}\text { Entire gastro- } \\
\text { intestinal } \\
\text { tract }\end{array}$ & $\begin{array}{l}\text { Colon and } \\
\text { rectum }\end{array}$ & Lungs & Lungs & Skin & Skin & Joints & Vascular wall & Adipose tissue \\
\hline $\begin{array}{l}\text { Predisposing } \\
\text { genetic } \\
\text { factors }\end{array}$ & $\begin{array}{l}\text { HLA-DQ2, } \\
\text { HLA-DQ8 }\end{array}$ & $\begin{array}{l}\text { NOD2; IBD } \\
\text { locus }\end{array}$ & $\begin{array}{l}\text { IBD locus; } \\
\text { MUC-3; HLA- } \\
\text { DR subtypes? }\end{array}$ & & $\begin{array}{l}\text { ADAM33; Th2 } \\
\text { gene cluster }\end{array}$ & Filaggrin & PSORS1 & $\begin{array}{l}\text { HLA-DR4 } \\
\text { subtypes }\end{array}$ & $\begin{array}{l}\text { Several incl. } \\
\text { ApoE4 }\end{array}$ & $\begin{array}{l}\text { Several } \\
\text { suggested }\end{array}$ \\
\hline $\begin{array}{l}\text { Triggering } \\
\text { factor(s) }\end{array}$ & Gluten peptides & $\begin{array}{l}\text { Commensal } \\
\text { gut } \\
\text { microbes }\end{array}$ & $\begin{array}{l}\text { Commensal gut } \\
\text { microbes }\end{array}$ & $\begin{array}{l}\text { Smoking; } \\
\text { irritants }\end{array}$ & $\begin{array}{l}\text { Allergens; } \\
\text { irritants }\end{array}$ & $\begin{array}{l}\text { Allergens; } \\
\text { irritants }\end{array}$ & $\begin{array}{l}\text { Skin } \\
\quad \text { streptococci }\end{array}$ & Not known & $\begin{array}{l}\text { Endothelial injury; } \\
\text { oxidised LDL }\end{array}$ & $\begin{array}{l}\text { Energy intake } \\
\text { in excess of } \\
\text { expenditure }\end{array}$ \\
\hline $\begin{array}{l}\text { Antigen(s) } \\
\text { involved }\end{array}$ & Gluten peptides & $\begin{array}{l}\text { Bacterial } \\
\text { antigens }\end{array}$ & $\begin{array}{l}\text { Bacterial } \\
\text { antigens }\end{array}$ & & Allergens & $\begin{array}{l}\text { Airborne } \\
\text { allergens; } \\
\text { Hom s } 4\end{array}$ & Streptococci & $\begin{array}{l}\text { Proteoglycans; } \\
\text { filaggrin }\end{array}$ & Micro-organisms & \\
\hline $\begin{array}{l}\text { Clinical } \\
\text { features }\end{array}$ & $\begin{array}{l}\text { Villus atrophy; } \\
\text { malabsorption }\end{array}$ & $\begin{array}{l}\text { Gut ulceration; } \\
\text { diarrhoea; } \\
\text { abdominal } \\
\text { pain; weight } \\
\text { loss; } \\
\text { malaise }\end{array}$ & $\begin{array}{l}\text { Gut ulceration; } \\
\text { bloody } \\
\text { diarrhoea; } \\
\text { abdominal } \\
\text { pain; urgency } \\
\text { to defecate }\end{array}$ & $\begin{array}{l}\text { Lung damage; } \\
\text { breathleness }\end{array}$ & $\begin{array}{l}\text { Wheeze; } \\
\text { mucus; } \\
\text { breathleness }\end{array}$ & Itchy, dry skin & Dry skin & $\begin{array}{l}\text { Joint swelling, } \\
\text { pain and } \\
\text { erosion }\end{array}$ & $\begin{array}{l}\text { Plaque formation } \\
\text { - ultimately MI, } \\
\text { stroke, etc., } \\
\text { due to plaque } \\
\text { rupture }\end{array}$ & $\begin{array}{l}\text { Weight gain; } \\
\text { insulin } \\
\text { resistance }\end{array}$ \\
\hline $\begin{array}{l}\text { Cells } \\
\text { involved }\end{array}$ & $\begin{array}{l}\text { Intraepithelial } \\
\text { lymphocytes; } \\
\text { epithelial cells; } \\
\text { gluten-specific } \\
\text { Th1 cells }\end{array}$ & Th1 cells & $\begin{array}{l}\text { Th1 and Th2 } \\
\text { cells (Th2 } \\
\text { dominant); } \\
\text { PMNs }\end{array}$ & PMNs & $\begin{array}{l}\text { Th2 cells; } \\
\text { mast cells; } \\
\text { eosinophils }\end{array}$ & $\begin{array}{l}\text { Th1 and Th2 } \\
\text { cells (Th2 } \\
\text { dominant); } \\
\text { mast cells; } \\
\text { eosinophils }\end{array}$ & $\begin{array}{l}\text { Th1 cells; NK } \\
\text { cells; PMNs; } \\
\text { keratino- } \\
\text { cytes }\end{array}$ & $\begin{array}{l}\text { Th1 cells; } \\
\text { fibroblasts; } \\
\text { B-cells }\end{array}$ & $\begin{array}{l}\text { Macrophages; } \\
\text { T-cells; plate- } \\
\text { lets; endothelial } \\
\text { cells }\end{array}$ & $\begin{array}{l}\text { Adipocytes; } \\
\text { macro- } \\
\text { phages }\end{array}$ \\
\hline $\begin{array}{l}\text { Mediators } \\
\text { involved }\end{array}$ & $\begin{array}{l}\text { IL-15, IFN- } \gamma \text {, } \\
\text { TNF- } \alpha\end{array}$ & $\begin{array}{l}\text { IL-12, TNF- } \alpha \\
\text { IL-6, PG, } \\
\text { MMP }\end{array}$ & $\begin{array}{r}\text { TNF- } \alpha, \text { IL-5, } \\
\text { IL-13, PG }\end{array}$ & MMPs & $\begin{array}{l}\text { IL-5, IL-13, } \\
\text { TNF- } \alpha \\
\text { cysLTs }\end{array}$ & $\begin{array}{l}\text { Th1 and Th2 } \\
\text { type } \\
\text { cytokines, } \\
\text { cysLTs, } \\
\text { allergen- } \\
\text { specific IgE }\end{array}$ & $\begin{array}{l}\text { Th1 type cyto- } \\
\text { kines, LTB4, } \\
\text { angiogenic } \\
\text { factors }\end{array}$ & $\begin{array}{l}\text { TNF- } \alpha, \text { IL-1, } \\
\text { IL-6, IL-17, } \\
\text { PG, LTB4, } \\
\text { MMP }\end{array}$ & $\begin{array}{l}\text { MCP-1 in plaque } \\
\text { formation; } \\
\text { MMP in plaque } \\
\text { rupture }\end{array}$ & MCP-1 \\
\hline Biomarkers & $\begin{array}{l}\text { Mucosal } \\
\text { architecture; } \\
\text { anti-gliadin } \\
\text { antibodies; } \\
\text { transglutaminase }\end{array}$ & $\begin{array}{l}\text { Mucosal } \\
\text { architecture; } \\
\text { endoscopic } \\
\text { scores; } \\
\text { faecal cal- } \\
\text { protectin; } \\
\text { clinical score } \\
\text { (CDAl); CRP }\end{array}$ & $\begin{array}{l}\text { Mucosal } \\
\text { architecture; } \\
\text { endoscopic } \\
\text { scores; faecal } \\
\text { calprotectin; } \\
\text { clinical score } \\
\text { (CAI); CRP }\end{array}$ & $\begin{array}{l}\text { Non-reversible } \\
\text { impaired } \\
\text { lung function } \\
\text { (low FEV1) }\end{array}$ & $\begin{array}{l}\text { Impaired but } \\
\text { reversible } \\
\text { lung function }\end{array}$ & Clinical score & $\begin{array}{l}\text { Clinical score; } \\
\text { CRP }\end{array}$ & $\begin{array}{l}\text { Rheumatoid } \\
\text { factor; } \\
\text { autoanti- } \\
\text { bodies; } \\
\text { X-ray; clinical } \\
\text { signs; CRP }\end{array}$ & $\begin{array}{l}\text { Angiography; } \\
\text { plasma lipids; } \\
\text { CRP; soluble } \\
\text { adhesion } \\
\text { molecules; } \\
\text { vessel wall } \\
\text { thickening (e.g. } \\
\text { intima-media } \\
\text { thickness); }\end{array}$ & $\begin{array}{l}\text { Body weight; } \\
\text { BMI; waist- } \\
\text { hip ratio; } \\
\text { body fatness }\end{array}$ \\
\hline
\end{tabular}

COPD, chronic obstructive pulmonary disease; HLA, human leucocyte antigen; NOD, nucleotide-binding oligomerisation domain; IBD, inflammatory bowel disease; Th, helper T-cell; PMN, polymorphonuclear cells; IFN, interferon; MMP, matrix metalloproteinase; cysLT, cysteinyl leukotriene; LT, leukotriene; MCP, monocyte chemoattractant protein; CDAl, Crohn's disease activity index; CRP, C-reactive protein; CAI, colitis activity index; FEV1, forced expiratory 


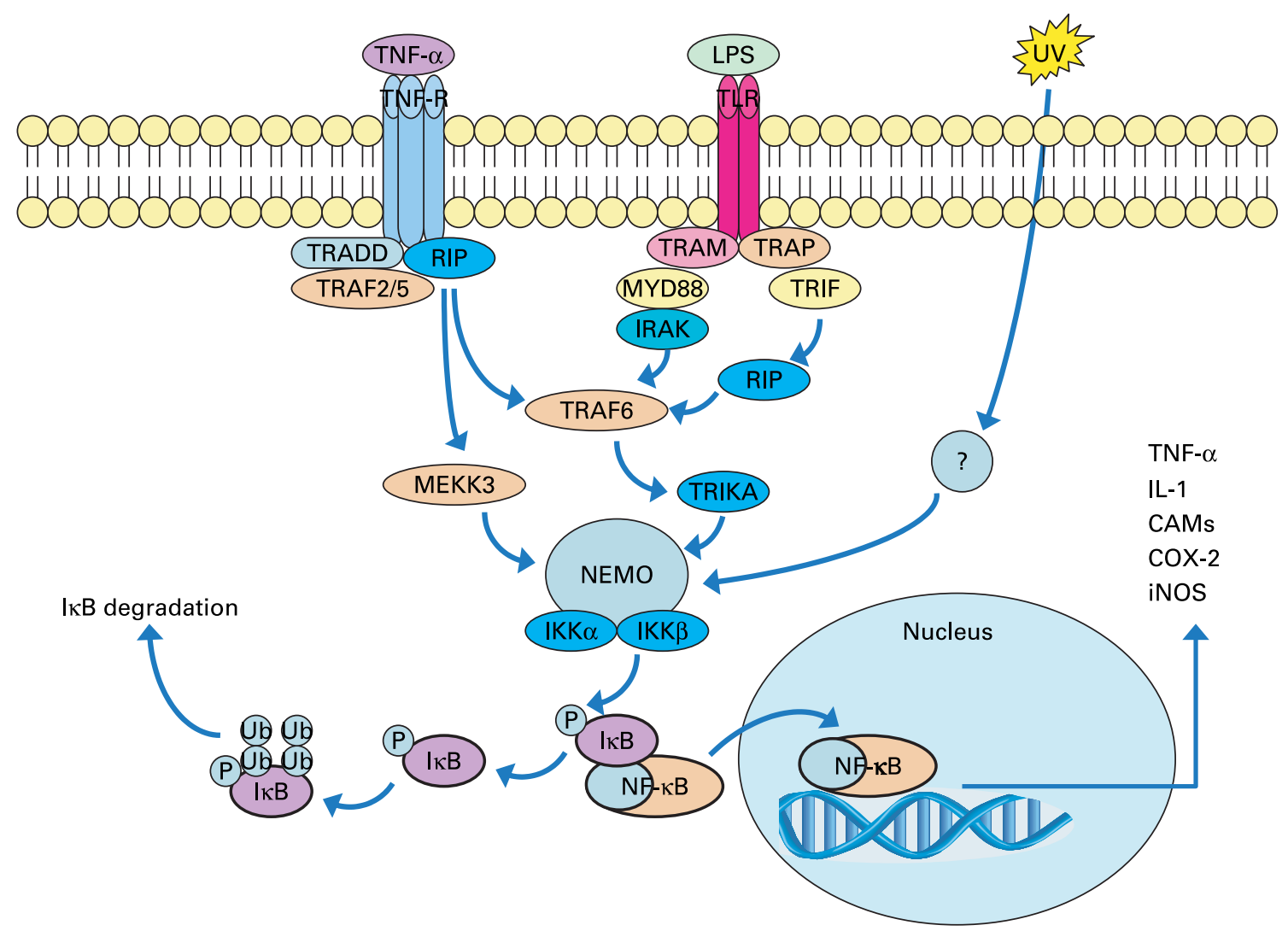

Fig. 3. NF-кB signalling pathway.

involve quite distinct antigens such as the gut microflora in $\mathrm{CD}$ and UC, fillagrin in eczema, and cartilage in RA. In all these cases, these responses seem to result from loss of barrier function and once initiated help to sustain the chronic inflammatory condition.

Why resolution of inflammation is absent or abnormal in so many pathophysiological processes remains largely unknown, although there are a number of mechanisms that may be considered.

(i) Persistent insult (i.e. chronic infection or continued exposure to initiating allergens) provides continued pro-inflammatory stimuli.

(ii) The inflammatory response results in tissue damage and loss of barrier function resulting in exposure of sequestered antigens and loss of tolerance to autoantigens or components of the microbiota, which then provide a trigger to drive prolonged inflammation.

(iii) Local (over)production of survival factors such as IL-5, granulocyte-macrophage colony stimulating factor and $\mathrm{IL}-1 \beta$ results in prolonged survival and activity of granulocytes.

(iv) Defective or reduced phagocytosis impairing clearance of cellular debris. This results in granulocyte necrosis and the release of reactive oxygen and nitrogen species as well as the activation of macrophages leading to sustained production of pro-inflammatory cytokines.

(v) Deficiency in immunological negative feedback mechanisms, which leads to loss of inflammatory control. This could be at the level of regulatory T-cell dysfunction (no release of anti-inflammatory cytokines), at the level of intracellular negative feedback mechanisms in immune cells or impaired macrophage suppresser activity (no release of regulatory proteins).

Although the relative importance may differ, these mechanisms seem to contribute to most of the conditions described here. Over time, chronic inflammation also results in a gradual overload with reactive oxidant species, which when insufficiently countered by the gradually weakened anti-oxidative defence systems results in a shift in redox balance. This in turn leads to uncontrolled activation of the redox-sensitive proinflammatory transcription factor $\mathrm{NF}-\kappa \mathrm{B}$, continued production of reactive oxygen and nitrogen species and supports chronic inflammation (Fig. 4).

The foregoing considerations suggest a number of potential targets for nutritional intervention, which are common across the various inflammatory pathologies considered here. Among these targets are common mediators, including ROS, arachidonic acid-derived lipid mediators (PG, LT), chemokines (IL-8, MCP-1), inflammatory cytokines (TNF- $\alpha$, IL-1, IL-6, IFN- $\gamma$, etc.) and matrix metalloproteinases, and common intracellular signalling factors including the transcription factors NF- $\mathrm{BB}$ and PPAR- $\gamma$ and their upstream regulators.

\section{Nutrition and inflammatory processes}

Fatty acids

Description, sources, normal intakes and roles. The general structure of a fatty acid is a hydocarbon (acyl) chain of varying 


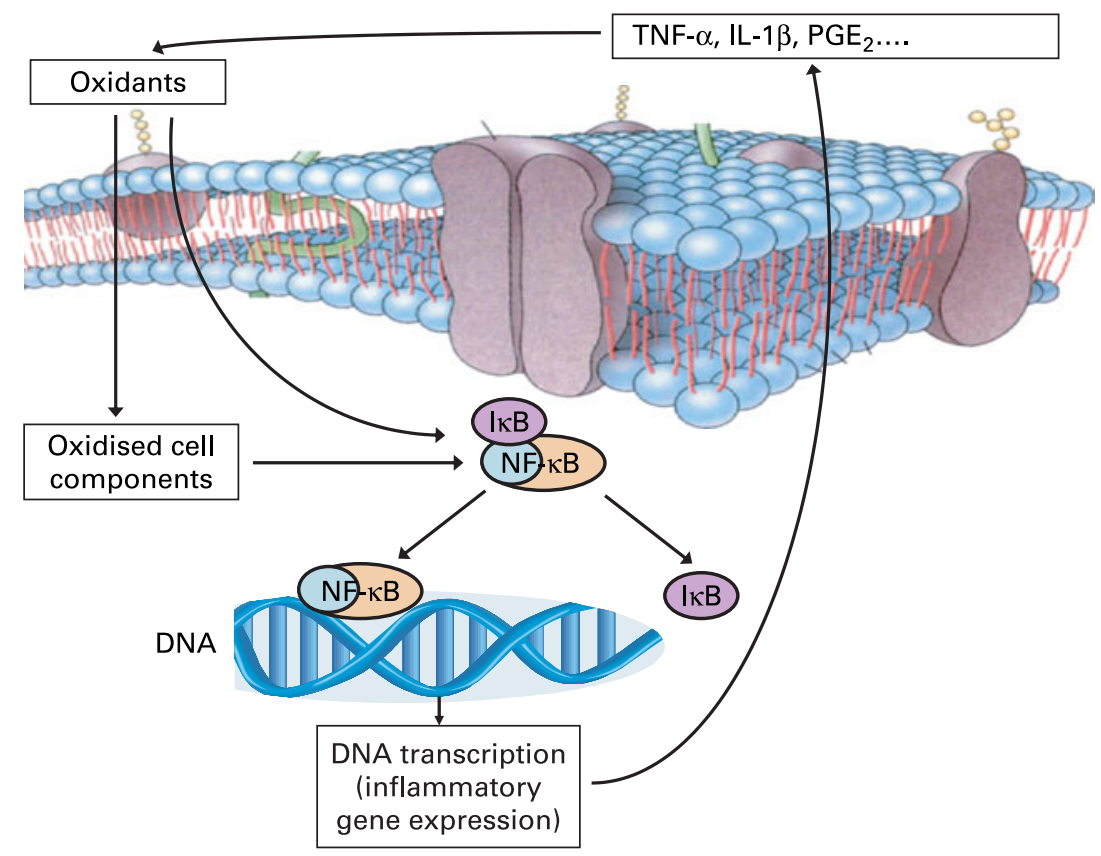

Fig. 4. Representation of the interaction between oxidant stress and inflammation. IкB, inhibitory subunit of NF-кB; IL, interleukin; NF-кB, nuclear factor $к \mathrm{~B}$; $P G$, prostaglandin; TNF, tumor necrosis factor.

length with a carboxyl group at one end and a methyl group at the other. The carboxyl group is reactive and readily forms ester links with alcohol groups for example those on glycerol or cholesterol. The most abundant fatty acids have straight chains of an even number of carbon atoms. Fatty acids containing double bonds are referred to as unsaturated fatty acids; a fatty acid containing two or more double bonds is called a PUFA. Most common unsaturated fatty acids contain cis rather than trans double bonds. However, trans double bonds do occur in ruminant fats (e.g. cow's milk), in plant lipids and in some seed oils. In some PUFA, the double bonds are not separated by a methylene $(-\mathrm{CH} 2-)$ group, but are conjugated. The systematic name for a fatty acid is derived from the number of carbon atoms in the acyl chain, the number of double bonds, the position of the double bonds (counting the carboxyl carbon as carbon 1) and their configuration (cis or trans). An alternative shorthand notation for fatty acids is frequently used. This relies upon identifying the number of carbon atoms in the chain, the number of double bonds and the position of the double bond closest to the methyl terminus of the acyl chain (the methyl carbon is known as the $n$-carbon). Thus, cis 9, cis 12-octadecadienoic acid is notated as 18:2n-6. In addition to these nomenclatures, fatty acids are often described by a common name ${ }^{(201)}$. Human subjects cannot synthesise the simplest $n-6$ and $n$-3 PUFA (linoleic acid (18:2n-6) and $\alpha$-linolenic acid (18:3n-3)) and so these are regarded as essential fatty acids.

Fatty acids in fats, oils and foodstuffs are mainly esterified to glycerol, as TAG, although some are present as esterified components of phospholipids, glycolipids and other lipids. With regard to inflammatory processes, most attention has been paid to the effects of $n$ ?6 and $n$-3 PUFA ${ }^{(173,202)}$, although other fatty acid classes including saturated and trans fatty acids may also influence inflammatory processes. Important dietary sources of the $n-6$ PUFA linoleic acid include vegetable oils (e.g. maize, sunflower, safflower and soyabean oils) and products made from those oils (e.g. margarines). Oils like soyabean also contain $\alpha$-linolenic acid; other good sources of this fatty acid include some nuts, flaxseeds and flaxseed (linseed) oil. Some seed oils contain moderate to high proportions of relatively unusual fatty acids (e.g. $\gamma$-linolenic acid $(18: 3 n-6)$ in borage (starflower) and evening primrose oils). Meat is an important source of the VLC $n-6$ fatty acid, arachidonic acid (20:4n-6). Fish can be classified into lean fish that store lipid as TAG in the liver (e.g. cod) or 'fatty' ('oily') fish that store lipid as TAG in the flesh (e.g. mackerel, herring, salmon, tuna, sardines). The oil obtained from fatty fish flesh or lean fish livers is termed 'fish oil' and it has the distinctive characteristic of being rich in the VLC $n$-3 PUFA eicosapentaenoic acid (EPA; $20: 5 n-3)$ and DHA (22:6n-3).

There are large differences in fat intake among countries with average intakes among adults varying from $<20 \mathrm{~g} / \mathrm{d}$ in some developing countries to $>100 \mathrm{~g} / \mathrm{d}$ in some developed countries. The mix of fatty acids consumed also varies in accordance with the fatty acid compositions of the fats and oils used in food preparation and of the foodstuffs eaten. Average fat consumption has changed over time and continues to do so. In many developing countries fat intake is increasing, while in developed countries fat intake has tended to decline over the last 40 years or so.

The type of fat consumed has also changed over time, meaning that the fatty acid composition of the human diet has changed. The latest figures for the UK indicate that adult males consume an average of $85 \mathrm{gfat} / \mathrm{d}$ and adult females $60 \mathrm{~g} / \mathrm{d}$ (both approx. $35 \%$ of dietary energy). The main PUFA in the diet is linoleic acid followed by $\alpha$-linolenic acid. On average, adult men in the UK consume about 12 and $1.9 \mathrm{~g}$ linoleic and $\alpha$-linolenic acids, respectively, per day. Adult women in the UK consume about 8.7 and $1.4 \mathrm{~g}$ linoleic and $\alpha$-linolenic acids, respectively, per day. Hulshof et al. ${ }^{(203)}$ 
provided details of intakes of fatty acids among 14 Western European countries, while Burdge \& Calder ${ }^{(204)}$ included some data from North America and Australia. Longer-chain PUFA are consumed in lower amounts than linoleic and $\alpha$-linolenic acids. Estimates of the intake of arachidonic acid in Western populations vary between 50 and $300 \mathrm{mg} / \mathrm{d}$ for adults $^{(201)}$. In the absence of fatty fish or fish oil consumption, $\alpha$-linolenic acid is by far the principal dietary $n-3$ PUFA. Average intake of the VLC $n-3$ PUFA in the UK and in other Western countries where oily fish consumption is not the norm is estimated at $<250 \mathrm{mg}$ per day ${ }^{(201)}$.

Fatty acids are transported in the bloodstream largely in esterified form as components of lipoproteins, although albumin-bound NEFA also circulate ${ }^{(205)}$. Fatty acids have many diverse functions in cells; their principal roles are as energy sources and as membrane constituents ${ }^{(201)}$. Many types of fatty acid can fill these roles. Certain fatty acids have additional, specific roles: arachidonic, dihomo- $\gamma$-linolenic (20:3n-6) and EPAs act as precursors for eicosanoid synthesis; myristic and palmitic acids may be covalently attached to certain proteins and this is believed to play an important role in the functioning of these proteins; fatty acids are able to influence intracellular signalling processes; fatty acids are able to influence transcription factor activity and gene expression. Through these different actions, fatty acids are able to influence cellular functions and so affect physiological responses, including inflammation.
Effects on inflammatory processes and mechanisms of action Arachidonic acid, arachidonic acid-derived eicosanoids and inflammation. The key link between fatty acids and inflammation is that eicosanoids that act as mediators and regulators of inflammation are generated from 20-carbon PUFA. Because inflammatory cells typically contain a high proportion of the n-6 PUFA arachidonic acid and low proportions of other 20carbon PUFA, arachidonic acid is usually the major substrate for eicosanoid synthesis. Eicosanoids, which include PG, thromboxanes, LT and other oxidised derivatives, are generated from arachidonic acid by reactions catalysed by cyclooxygenase (COX) and lipoxygenase (LOX) enzymes (Fig. 5). There are at least two COX enzymes and several LOX enzymes that are expressed in different cell types, according to different conditions and which between them produce a range of mediators involved in modulating the intensity and duration of inflammatory responses ${ }^{(206,207)}$. These mediators have cell- and stimulus-specific sources and frequently have opposing effects. Thus, the overall physiological (or pathophysiological) outcome will depend upon the cells present, the nature of the stimulus, the timing of eicosanoid generation, the concentrations of different eicosanoids generated and the sensitivity of target cells and tissues to the eicosanoids generated.

The amount of arachidonic acid in inflammatory cells can be increased by including it in the $\operatorname{diet}^{(173)}$ and may also be influenced by the dietary intake of its precursor, linoleic

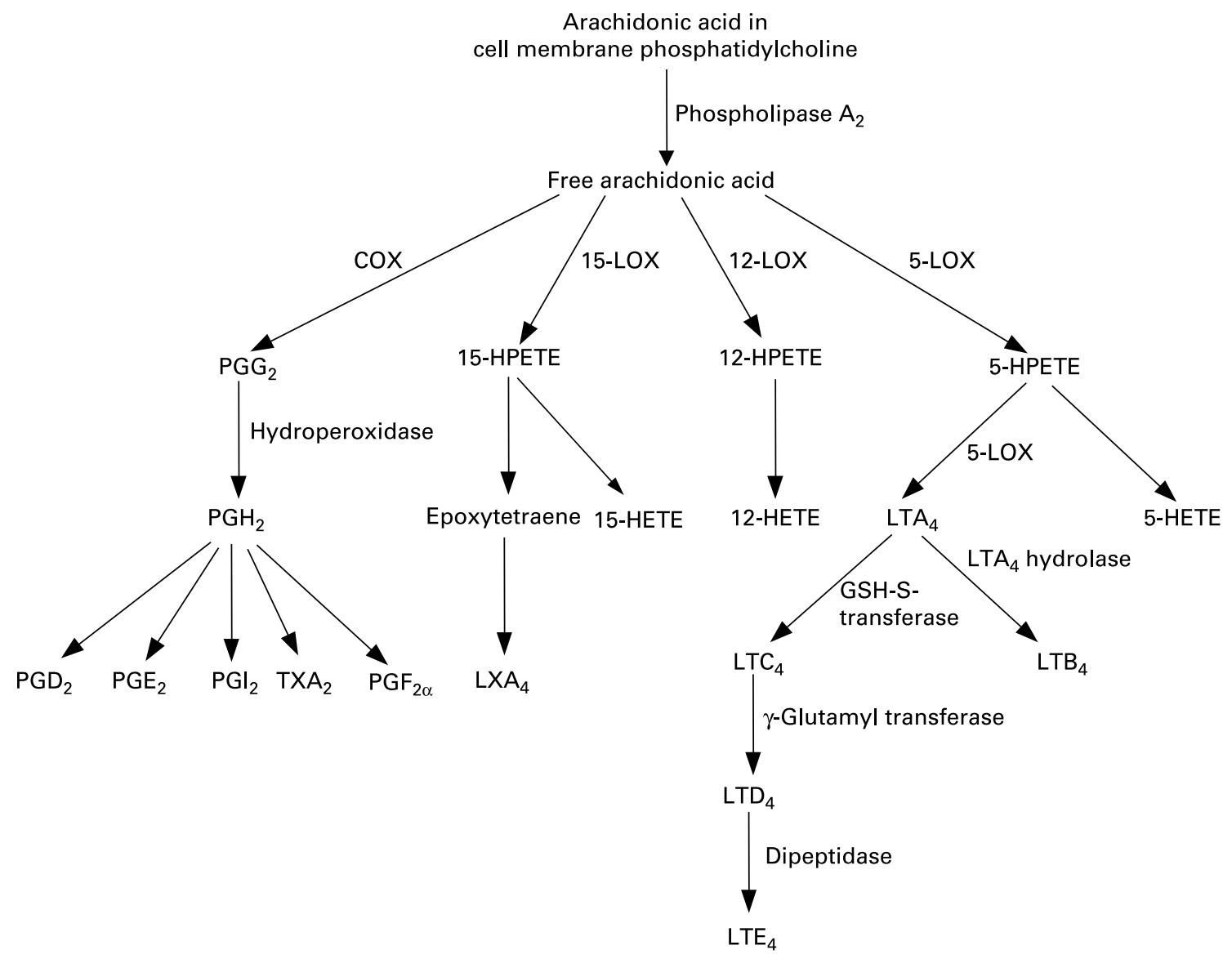

Fig. 5. Eicosanoid biosynthesis from arachidonic acid. COX, cyclo-oxygenase; HETE, hydroxyeicosatetraenoic acid; HPETE, hydroperoxyeicosatetraenoic acid; LOX, lipoxygenase; LT, leukotriene; PG, prostaglandin; TX, thromboxane. 
acid, although the range of linoleic acid intake over which this relationship occurs has not been defined for human subjects. The role of arachidonic acid as a precursor for the synthesis of eicosanoids indicates the potential for dietary n-6 PUFA (linoleic or arachidonic acids) to influence inflammatory processes. However, this has been little investigated in human settings. Supplementation of the diet of healthy young male subjects with $1.5 \mathrm{~g}$ arachidonic acid/d for 7 weeks resulted in a marked increase in the production of PGE2 and LTB4 by endotoxin-stimulated mononuclear cells ${ }^{(208)}$. However, production of TNF- $\alpha$, IL- $1 \beta$ and IL- 6 by the latter was not significantly altered. Thus, increased arachidonic acid intake may result in changes indicative of selectively increased inflammation or inflammatory responses in human subjects. Supplementation of the diet of healthy elderly subjects with arachidonic acid $(0.7 \mathrm{~g} / \mathrm{d}$ in addition to a habitual intake of about $0.15 \mathrm{~g} / \mathrm{d}$ ) for 12 weeks did not affect endotoxinstimulated production of TNF- $\alpha$, IL- $1 \beta$ or IL- 6 by mononuclear cells, did not alter superoxide production by neutrophils or monocytes and did not alter plasma-soluble adhesion molecule concentrations $^{(209)}$.

Very long-chain n-3 PUFA and inflammatory eicosanoid production. Increased consumption VLC $n-3$ PUFA such as EPA and DHA, usually given as fish oil in experimemtal settings, results in increased proportions of those fatty acids in inflammatory cell phospholipids (see Calder ${ }^{(173)}$ for references). This occurs in a dose-response fashion and is partly at the expense of arachidonic acid ${ }^{(210,211)}$. Since there is less substrate available for the synthesis of eicosanoids from arachidonic acid, fish oil supplementation of the human diet has been shown to result in decreased production of these mediators by inflammatory cells ${ }^{(173)}$. Although most studies have used fish oil, Kelley et al. ${ }^{(212)}$ demonstrated that $6 \mathrm{~g}$ DHA/d resulted in decreased production of PGE2 and LTB4 by endotoxin-stimulated mononuclear cells.

EPA is also able to act as a substrate for both COX and 5-LOX, giving rise to eicosanoids with a slightly different structure to those formed from arachidonic acid. Thus, fish oil supplementation of the human diet has been shown to result in increased production of alternative eicosanoids (see Calder ${ }^{(173)}$ for references). The functional significance of this is that the mediators formed from EPA are believed to be less potent than those formed from arachidonic acid (see Calder ${ }^{(173)}$ for references).

In addition to VLC $n$ - 3 PUFA modulating the generation of eicosanoids from arachidonic acid and to EPA acting as substrate for the generation of alternative eicosanoids, recent studies have identified a novel group of mediators, termed E- and D-series resolvins, formed from EPA and DHA, respectively, by the sequential actions of COX-2 and LOX enzymes, which appear to exert anti-inflammatory and inflammation resolving actions (see Serhan et al. ${ }^{(213)}$ and Serhan ${ }^{(214)}$ for reviews).

Anti-inflammatory effects of very long-chain n-3 PUFA other than altered eicosanoid production. A number of dietary supplementation studies that used between $3 \cdot 1$ and $14.4 \mathrm{gEPA}+\mathrm{DHA} / \mathrm{d}$ have demonstrated a time-dependent decrease in chemotaxis of human neutrophils and monocytes towards various chemoattractants; both the distance of cell migration and the number of cells migrating were decreased (see Calder ${ }^{(173)}$ for references).
Cell culture and animal feeding studies report decreased expression of some adhesion molecules on the surface of monocytes, macrophages or endothelial cells following exposure to VLC $n-3$ PUFA (see Calder ${ }^{(173)}$ for references). Supplementing the diet of healthy human subjects with fish oil providing about $1.5 \mathrm{~g}$ EPA + DHA/d resulted in a lower level of expression of ICAM-1 on the surface of blood monocytes stimulated ex vivo with IFN- $\gamma^{(215)}$. Dietary fish oil providing $1 \cdot 1 \mathrm{gEPA}+\mathrm{DHA} / \mathrm{d}$ was found to decrease circulating levels of soluble VCAM-1 in elderly subjects ${ }^{(216)}$, but it is not clear whether this represents decreased surface expression of VCAM-1.

Supplementation studies providing 3.1-8.4 g EPA + DHA/ $\mathrm{d}$ have reported decreases in the production of ROS (superoxide and/or hydrogen peroxide) by stimulated human neutrophils and monocytes (see Calder ${ }^{(173)}$ for references). A recent study has reported the dose-response relationship between EPA intake and decreased superoxide production by human neutrophils and identified that cells from older subjects seem to be more sensitive to EPA than those from young subjects $^{(211)}$.

Cell culture studies demonstrate that EPA and DHA can inhibit the production of inflammatory cytokines (TNF- $\alpha$, IL-1 $\beta$, IL-6, IL-8) by monocytes and endothelial cells (see Calder ${ }^{(173)}$. Fish oil feeding decreased ex vivo production of TNF- $\alpha$, IL-1 $\beta$ and IL-6 by rodent macrophages (see Calder ${ }^{(173)}$ ). Supplementation of the diet of healthy human volunteers with fish oil providing more than $2 \mathrm{~g} \mathrm{EPA}+$ DHA/d has been shown to decrease production of TNF, or IL-1 or IL-6 by mononuclear cells in some studies (see Calder ${ }^{(173)}$ ). Caughey et al. ${ }^{(217)}$ reported a significant inverse correlation between the EPA content of mononuclear cells and the ability of those cells to produce TNF- $\alpha$ and IL- $1 \beta$ in response to endotoxin. Kelley et al. ${ }^{(212)}$ showed that $6 \mathrm{~g} \mathrm{DHA} / \mathrm{d}$ for 12 weeks resulted in decreased production of TNF- $\alpha$ and IL-1 $\beta$ by endotoxinstimulated mononuclear cells. Both EPA and DHA $(4 \mathrm{~g} / \mathrm{d}$ for 6 weeks) decreased plasma TNF- $\alpha$ concentrations in type 2 diabetics, although DHA was more potent ${ }^{(218)}$. It should be noted that there are also several studies that fail to show effects of dietary long-chain $n$-3 PUFA on production of inflammatory cytokines in human subjects (see Calder ${ }^{(173,202)}$ for references and discussion).

$\alpha$-Linolenic acid and inflammatory processes. Increased consumption of $\alpha$-linolenic acid results in an increased content of EPA and docosapentaenoic acid in the membranes of inflammatory cells (see Burdge \& Calder ${ }^{(219)}$ for references) in parallel with a decreased content of arachidonic acid. This might be expected to affect inflammation. A recent study has reported an inverse association between $\alpha$-linolenic acid intake and plasma concentrations of IL- 6 and sVCAM- 1 but not of CRP, soluble TNF receptor-2, SE-selectin or sICAM-1 in samples taken from the Nurses Health Study 1 cohort $^{(22)}$. After adjustment for age, BMI, physical activity, smoking status, alcohol consumption and intakes of linoleic acid and SFA, there were significant negative associations between $\alpha$-linolenic acid intake and plasma CRP, IL-6 and E-selectin concentrations ${ }^{(220)}$. These observations would suggest that increasing $\alpha$-linolenic acid intake would result in decreased inflammation. Caughey et al. ${ }^{(217)}$ reported that $13.7 \mathrm{~g} / \mathrm{d} \alpha$-linolenic acid for 4 weeks resulted in a decrease in ex vivo production of PGE2, TNF- $\alpha$ and IL-1 $\beta$ by 
endotoxin-stimulated mononuclear cells by 33, 27 and $30 \%$, respectively. By comparison, fish oil providing $2.7 \mathrm{~g} / \mathrm{d}$ EPA + DHA decreased the production of these inflammatory mediators by 55,70 and $78 \%$, respectively ${ }^{(217)}$. Thus, on a g/d basis, VLC $n-3$ PUFA are about eight to fourteen times more potent than $\alpha$-linolenic acid with respect to this outcome in healthy subjects. In contrast to the observations of Caughey et al. ${ }^{(217)}$, several studies using lower intakes of $\alpha$-linolenic acid $(2-9.5 \mathrm{~g} / \mathrm{d})$ did not find effects on neutrophil chemotaxis, neutrophil respiratory burst, monocyte respiratory burst, TNF- $\alpha$, IL- $1 \beta$ and IL- 6 production by endotoxin-stimulated mononuclear cells, all studied ex vivo, or on soluble adhesion molecule concentrations (see Burdge \& Calder ${ }^{(219)}$ for references). Furthermore, a study by Rallidis et al. ${ }^{(221)}$ reported no effect of $8 \mathrm{~g} / \mathrm{d} \alpha$-linolenic acid on sICAM-1 or SE-selectin concentrations and a similar decrease in sVCAM-1 concentration in both $\alpha$-linolenic acid and control groups. Likewise, Bemelmans et al. ${ }^{(22)}$ found no effect of $6 \cdot 3 \mathrm{~g} / \mathrm{d} \alpha$-linolenic acid on sICAM-1 concentration at 1 and 2 years of intervention. Taken together, these data suggest that increasing $\alpha$-linolenic acid intake to $>9 \mathrm{~g} / \mathrm{d}$ is required in order for marked anti-inflammatory effects to be seen. Even then, the effects will be much more modest than those exerted by VLC $n-3$ PUFA $^{(217)}$. However, both Rallidis et al. ${ }^{(223)}$ and Bemelmans et al. ${ }^{(22)}$ did find a significant decrease in CRP concentration, suggesting that this may be a marker that is more sensitive to the intakes of $\alpha$-linolenic acid, which do not affect soluble adhesion molecule or cytokine concentrations or ex vivo inflammatory cell responses. One study using a very high intake of $\alpha$-linolenic acid (approx. $17.5 \mathrm{~g} / \mathrm{d}$ ) reported significant decreases in both CRP and soluble adhesion molecule concentrations $^{(224)}$. Interestingly, the authors found that the changes in these inflammatory markers were significantly related to changes in serum concentrations of EPA or EPA plus docosapentaenoic acid, but not of $\alpha$-linolenic acid. This suggests that the observed effects are due to the VLC $n-3$ PUFA rather than to $\alpha$-linolenic acid per se. Thus, the likely explanation for the lack of anti-inflammatory effect of $\alpha$-linolenic acid at modest and even at rather high intakes is that there has been insufficient conversion to the more active EPA.

Mechanisms of action. It is clear that one anti-inflammatory mechanism of action of VLC $n-3$ PUFA is antagonism of production of inflammatory eicosanoids from arachidonic acid coupled with the generation of less potent EPA-derived eicosanoids and, in some conditions, anti-inflammatory resolvins $^{(225)}$. Altered eicosanoid profiles may have downstream effects since some eicosanoids regulate production of inflammatory cytokines. Thus, an n-3 PUFA-induced decrease in eicosanoid production might affect production of TNF- $\alpha$, IL-1 $\beta$, IL-6, etc. However, the effects of $n-3$ PUFA on inflammatory cytokine production and on some other inflammatory processes appear to be eicosanoid independent. One alternative candidate mechanism of action is altered activation of transcription factors involved in inducing transcription of inflammatory genes (e.g. NF-кB, PPAR- $\gamma$; see Calder ${ }^{(226,227)}$, which may occur as a result of altered plasma membrane signalling processes ${ }^{(228-231)}$.

Effects on clinical outcomes in inflammatory conditions Inflammatory bowel diseases. Dietary fish oil shows improvements in animal models of IBD (see Calder ${ }^{(173)}$ ).
Shoda et al. ${ }^{(232)}$ determined that the increased incidence of $\mathrm{CD}$ in Japan was significantly associated with an increase in the ratio of $n-6$ to $n-3$ PUFA in the diet. They suggest that a diet high in $n-6$ PUFA relative to $n-3$ PUFA somehow plays a causal role in the disease, and that an increase in $n-3$ PUFA intake may be of benefit. VLC $n-3$ PUFA are incorporated into gut mucosal tissue of patients with IBD, who supplement their diet with fish oil and there are reports that this results in anti-inflammatory effects, such as decreased LTB4 production by neutrophils and colonic mucosa, decreased PGE2 and TXB2 production by colonic mucosa and decreased production of PGE2 by blood mononuclear cells (see Calder ${ }^{(173)}$ for references). Small open-label or pilot studies reported clinical benefit of fish oil supplementation in UC (see Calder ${ }^{(173)}$ ). A number of randomised, placebocontrolled, double-blind studies of fish oil in IBD have been reported (see Calder ${ }^{(173)}$ for details). The dose of VLC $n-3$ PUFA used in these trials was between 2.7 and $5.6 \mathrm{~g} / \mathrm{d}$ and averaged about $4.5 \mathrm{~g} / \mathrm{d}$. Some of these trials indicate benefits of fish oil, which include improved clinical score, improved gut mucosal histology, improved sigmoidoscopic score, lower rate of relapse and decreased use of corticosteroids. One study of special note is that of Belluzzi et al. ${ }^{(233)}$ in which patients with $\mathrm{CD}$ in remission were randomised to receive placebo or $2.7 \mathrm{~g}$ VLC $n-3$ PUFA/d from an enterically coated fish oil preparation for 1 year: there was a significant difference in the proportion of patients who relapsed over 12 months: $28 \%$ in the fish oil group v. $69 \%$ in the placebo group. Reviews of trials of fish oil in IBD conclude that there is some benefit from fish oil in IBD. A recent metaanalysis has concluded that there may be reduction in requirement for corticosteroids ${ }^{(234)}$.

Asthma. There are epidemiologic data linking high $n-6$ PUFA or low $n-3$ PUFA consumption with childhood asthma and allergic conditions (see Calder ${ }^{(173)}$ for references). Early exposure to VLC $n$-3 PUFA does appear to alter cytokine production by neonatal T-cells ${ }^{(235,236)}$ although the longer-term clinical impact of this is not yet clear. Several studies report anti-inflammatory effects of fish oil in patients with asthma, such as decreased four-series LT production and leucocyte chemotaxis (see Calder ${ }^{(173)}$ for references). A number of randomised, placebo-controlled, double-blind studies of fish oil in asthma have been reported (see Calder ${ }^{(173)}$ for details). A systematic review concluded that there was no consistent effect on forced expiratory volume at one second, peak flow rate, asthma symptoms, asthma medication use or bronchia hyper-reactivity ${ }^{(237)}$. However, one study in children has shown improved peak flow and reduced asthma medication use with fish oil ${ }^{(238)}$. A more recent report covering twenty-six studies (both randomised, placebo-controlled and others) has concluded that no definitive conclusion can yet be drawn regarding the efficacy of VLC $n-3$ fatty acid supplementation as a treatment for asthma in children and adults ${ }^{(239)}$. However, studies by Broughton et al. ${ }^{(240)}$ and Nagakura et al. ${ }^{(238)}$ indicate that there may be subgroups of asthmatic subjects who may benefit greatly from VLC $n$-3 PUFA. A recent study has reported the effects of a high level of fish oil, providing $5.2 \mathrm{~g} / \mathrm{d}$ EPA + DHA, on sputum inflammatory cytokines and pulmonary function in exercise-induced bronchoconstriction ${ }^{(68)}$. Concentrations of TNF- $\alpha$ and IL-1 $\beta$ in sputum supernatant were significantly 
lower in the fish oil group and this change was accompanied with improved pulmonary function.

Chronic obstructive pulmonary disease. Two recent studies have investigated the effects of fairly low supplemental intake of EPA + DHA on inflammatory markers, as well as exercise capacity, in COPD $^{(241,242)}$. Broekhuizen et al. ${ }^{(241)}$ reported improved exercise capacity in a cycling test but no effect on systemic levels of IL-6 and TNF- $\alpha$ following intake of a supplement containing $1.0 \mathrm{~g} / \mathrm{d}$ EPA + DHA. Matsuyama et al. ${ }^{(242)}$ measured both systemic (serum) and sputum TNF- $\alpha$ and IL-8 levels in a 2-year intervention with a supplemental intake of $0.6 \mathrm{~g} / \mathrm{d} \mathrm{EPA}+\mathrm{DHA}$. The present study also failed to find differences in serum cytokine concentrations, but did report decreased sputum TNF- $\alpha$ and IL-8 levels in the $n-3$ PUFA group. This effect was accompanied by improved exercise capacity in a walk test.

Rheumatoid arthritis. Dietary fish oil shows improvements in animal models of RA (see Calder ${ }^{(173)}$ ). Several studies report anti-inflammatory effects of fish oil in patients with RA, such as decreased LTB4 production by neutrophils and monocytes, decreased IL-1 production by monocytes, decreased plasma IL-1 $\beta$ concentrations, decreased serum CRP concentrations and normalisation of the neutrophil chemotactic response (see Calder ${ }^{(173)}$ for references). A number of randomised, placebo-controlled, double-blind studies of fish oil in RA have been reported (see Calder ${ }^{(173)}$ for details). The dose of VLC $n-3$ PUFA used in these trials was between 1.6 and $7.1 \mathrm{~g} / \mathrm{d}$ and averaged about $3.5 \mathrm{~g} / \mathrm{d}$. Almost all of these trials showed some benefit of fish oil, including reduced duration of morning stiffness, reduced number of tender or swollen joints, reduced joint pain, reduced time to fatigue, increased grip strength and decreased use of non-steroidal anti-inflammatory drugs. One study reported greater efficacy of fish oil against a background diet designed to be low in arachidonic acid ${ }^{(243)}$. Reviews of the trials of fish oil in RA have concluded that there is benefit and a meta-analysis concluded that dietary fish oil supplementation significantly reduces tender joint count and morning stiffness ${ }^{(24)}$. A recent metaanalysis has concluded that VLC $n-3$ fatty acids may reduce requirements for corticosteroids ${ }^{(234)}$. Thus, there is reasonably strong evidence that VLC $n$-3 PUFA have some clinical benefits in RA.

Psoriasis. Intravenous fish oil led to resolution of psoriasis in one study ${ }^{(245)}$. Dietary supplementation studies with fish oil do not present a clear picture although some of these have reported clinical benefit (see $\mathrm{Ziboh}^{(246)}$ for a review).

$U V$-induced skin inflammation. In experimental studies of skin inflammation, UV-induced dose-response curves were generated before and after 3 months of dietary supplementation with fish oil ${ }^{(247)}$. There was a significant inhibition of the erythemal response, reduction in PGE2 release and biochemical analysis of skin biopsies, which showed that the VLC $n$-3 PUFA were acting as a scavenger for reactive oxygen ${ }^{(247,248)}$.

$C V D$. Substantial evidence from epidemiological and case-control studies indicates that consumption of fish, fatty fish and VLC $n$-3 PUFA reduces risk of cardiovascular mortality ${ }^{(177,178,249,250)}$. Secondary prevention studies using VLC $n-3$ PUFA in myocardial infarction survivors have shown a reduction in total mortality and cardiovascular mortality with an especially potent effect on sudden death ${ }^{(175)}$. This protective effect of $n-3$ PUFA towards sudden death was apparent within 4 months of beginning supplementation ${ }^{(176)}$. VLC $n-3$ PUFA have been shown to influence a number of CVD risk factors (see Kris-Etherton et al. ${ }^{(249)}$ and Calder ${ }^{(250)}$ for references), and the extent to which a reduction in inflammation protects against the growth of the atherosclerotic plaque and decreases the risk and severity of cardiovascular events is not clear. However, one study suggests that VLC $n-3$ PUFA might act to stabilise advanced atherosclerotic plaques, perhaps through their anti-inflammatory effects ${ }^{(174)}$.

\section{Antioxidant vitamins ( $C$ and $E, \beta$-carotene)}

Oxidative stress and inflammation. There is a strong interaction between oxidative stress and inflammation. The generation of oxidants (e.g. superoxide radicals, hydrogen peroxide), for example by granulocytes exposed to bacterial cell wall peptides or lipopolysaccharides, is part of the host inflammatory response. Oxidants can damage components of host cells, such as the PUFA in cell membranes. In turn, oxidants and oxidised cell components, acting through transcription factors such as $\mathrm{NF}-\kappa \mathrm{B}$, induce production of inflammatory eicosanoids and cytokines (Fig. 4). Thus, one mechanism to diminish inflammatory mediator production may be to prevent oxidative stress. This is accomplished through enhancing antioxidant defence mechanisms. In order to monitor antioxidant defence, individual components of that defence can be measured (e.g. vitamin C, vitamin E, antioxidant enzyme activities). Additionally, the effectiveness of the non-enzymatic endogenous antioxidant network can be assessed measuring total antioxidant capacity (TAC), defined as the moles of oxidants neutralised by 1 litre of the tested sample ${ }^{(251)}$. TAC considers the cumulative action of all the antioxidants present in the matrix (plasma, saliva, diet, etc.), thus providing an integrated parameter rather than the simple summary of measurable antioxidants, giving an insight into the delicate balance between antioxidants molecules. Endogenous TAC has been shown to be modulated by dietary ingestion of plant food ${ }^{(252)}$ and to decline in subjects affected by $\mathrm{CVD}^{(253)}$ and cancer ${ }^{(254)}$. Population-based case-control studies have shown an inverse association of the TAC of the diet with risk of gastric cancer ${ }^{(255)}$ and overall mortality ${ }^{(256)}$ Despite these promising results, evidence about the role of the dietary or endogenous TAC and inflammation is lacking. Recently, TAC has been shown to be inversely and independently associated with plasma levels of CRP in 243 non-diabetic subjects $^{(257)}$. This observation highlights the interrelationship between oxidative stress and inflammation and suggests that improving TAC by dietary means may be a fruitful anti-inflammatory approach.

\section{Vitamin $C$}

Description, sources, normal intakes and roles. The term vitamin $\mathrm{C}$ includes ascorbic acid (AA) and dehydroascorbic acid, both of which exhibit anti-scorbutic activity. AA, the enolic form of an $\alpha$-ketolactone, is the functional and primary in vivo form of vitamin C. AA contains an asymmetric carbon atom that allows two enantiomeric forms, of which the L-form is naturally occurring. The two enolic hydrogen atoms of AA provide the electrons for its function as a reductant and antioxidant. The one-electron oxidation product of AA is the ascorbyl radical. It readily dismutates to 
AA and dehydroascorbic acid, the two-electron oxidation product. Both ascorbyl radical and dehydroascorbic acid are readily reduced back to AA. Dehydroascorbic acid can also be irreversibly hydrolysed to 2,3-diketo-L-gulonic acid, which is further decarboxylated and degraded to oxalic acid and threonic acid.

Vitamin $\mathrm{C}$ is essential for human subjects and a few other mammals, including guinea pigs and monkeys, because they are unable to synthesise AA from glucose due to lack of the enzyme gulonolactone oxidase. The main dietary sources of vitamin $\mathrm{C}$ in the typical Western diet are fruits and vegetables, including fruit and vegetable juices, as well as potatoes. Black currant, raw pepper, raw broccoli, kiwi, strawberries, oranges and freshly squeezed orange juice are particularly rich in vitamin $\mathrm{C}$

Present dietary reference intakes have been set at 90 and $75 \mathrm{mg} / \mathrm{d}$ for adult males and females, respectively ${ }^{(258)}$. An additional $35 \mathrm{mg} / \mathrm{d}$ is recommended for smokers, given the greater metabolic turnover of vitamin $\mathrm{C}$ in smokers. The recommended five servings per day of fruits and vegetables should provide at least $200 \mathrm{mg}$ of vitamin C. Storage and food processing have a major impact on vitamin $\mathrm{C}$ content. There are also an increasing number of foods and drinks fortified with vitamin $\mathrm{C}$. At usual dietary vitamin $\mathrm{C}$ intakes, in the range of $30-180 \mathrm{mg} / \mathrm{d}$, approximately $70-90 \%$ is absorbed, while absorption decreases to about $\leq 50 \%$ at doses exceeding $1 \mathrm{~g}^{(259)}$.

Maximal neutrophil concentrations with minimal urinary losses are thought to be the best biomarkers for estimating vitamin $C$ requirements. Saturation of leucocytes with vitamin $\mathrm{C}$ occurs at a lower intake compared with plasma ${ }^{(259)}$. Mean plasma concentrations of healthy subjects are about $65 \mu \mathrm{mol} / \mathrm{l}$, while scurvy occurs at plasma concentrations of less than $10 \mu \mathrm{mol} / \mathrm{l}$.

Vitamin C plays an important role in mechanisms involved in immune function and inflammatory processes, including, for instance, free radical scavenging and protection against lipid peroxidation. Given its high reducing power, vitamin $\mathrm{C}$ is a potent water-soluble antioxidant that acts in the aqueous phase both intra- and extracellularly. It readily scavenges ROS and reactive nitrogen species including hydroxyl, peroxyl, superoxide, nitroxide radical, peroxynitrite, as well as singlet oxygen and hypochlorite ${ }^{(260)}$. Ascorbyl radical, the one-electron oxidation product, and dehydroascorbic acid, the two-electron oxidation product of vitamin $\mathrm{C}$, are readily regenerated to $\mathrm{AA}$ by glutathione and NADH or NADPH.

AA tissue levels are relatively high and thus ensure efficient antioxidant protection, for instance, against ROS produced in activated neutrophils during phagocytosis ${ }^{(261)}$. AA also scavenges ROS in the aqueous phase before they initiate lipid peroxidation of fatty acids in plasma and $\operatorname{LDL}^{(262,263)}$. Moreover, AA enhances antioxidant protection by sparing or regenerating $\alpha$-tocopherol and glutathione back to their active forms ${ }^{(264)}$.

In addition to its antioxidant action, vitamin $\mathrm{C}$ is a cofactor for enzymes involved in the biosynthesis of collagen, carnitine and neurotransmitters as well as corticosteroids, the microsomal drug-metabolising enzymes and cytochrome P-450 electron transport. It modulates iron absorption, transport and storage. AA has also been shown to modulate PG synthesis ${ }^{(265)}$.
Effects on inflammatory processes and mechanisms of action. In infectious diseases and inflammation, ROS are generated during neutrophil activation and phagocytosis. AA exerts cellular protection against ROS generated by the respiratory burst. It neutralises hypochlorous acid, a powerful ROS generated by myeloperoxidase in activated neutrophils and monocytes such that inactivation of $\alpha 1$-proteinase inhibitor by hypochlorous acid is inhibited and, consequently, proteolytic damage is prevented at the site of inflammation ${ }^{(266)}$. It is important to note that, under normal conditions, phagocyte-derived ROS are neutralised without inhibiting the bactericidal activity of the phagosome ${ }^{(267)}$. Vitamin C also affects antimicrobial and natural killer cell activities, lymphocyte proliferation, chemotaxis and delayed-type hypersensitivity. Vitamin $C$ further enhances phagocytosis and migration of neutrophils ${ }^{(268)}$. In healthy subjects, vitamin $C$ concentrations are inversely correlated with CRP and 8-epi PGF2 $\alpha^{(269,270)}$. Plasma 8-epi PGF $2 \alpha$ and MCP-1 concentrations decreased significantly during consumption of approximately $72 \mathrm{mg} / \mathrm{d}$ vitamin $C$ from vegetable soup ${ }^{(269)}$.

An energy-dependent transport system allows for a 25- to 80 -fold accumulation of AA in neutrophils, lymphocytes and mast cells as compared with plasma levels ${ }^{(259,271)}$. During the course of infections, there is a rapid depletion of cellular ascorbate that returns to normal after recovery. At the site of inflammation, the ratio of oxidised to reduced ascorbate (dehydroascorbic acid: AA) is increased, as has been shown for the synovial fluid of RA joints ${ }^{(272)}$, and in the epithelial lining fluid of the lung and in the plasma of patients with adult respiratory distress syndrome ${ }^{(273)}$. Dehydroascorbic acid is the form of vitamin $\mathrm{C}$ that crosses the cell membranes and is then reduced intracellularly to AA. Reduction in vitamin $\mathrm{C}$ is associated with oxidation of reduced glutathione $(\mathrm{GSH})$ to its oxidised form (GSSG).

\section{Effects in inflammatory conditions}

Inflammatory bowel diseases. Serum and leucocyte ascorbate levels are low in adult and pediatric patients with CD, both in active disease and in disease remission ${ }^{(274-277)}$. Colonic biopsies of inflamed $v$. non-inflamed areas in patients with CD showed a $35 \%$ reduction in reduced and total AA content and in patients with UC total AA content was reduced by $73 \%$ and reduced AA by $41 \%{ }^{(278)}$. Also, different biomarkers of lipid peroxidation are higher ${ }^{(277)}$ and decreased during supplementation of vitamin $\mathrm{C}$ and $\mathrm{E}^{(279)}$. Fish oil plus antioxidant treatment $(90 \mathrm{mg}$ vitamin $\mathrm{C}, 30 \mathrm{mg}$ vitamin $\mathrm{E}, 150 \mu \mathrm{g}$ retinol equivalents as $\beta$-carotene) of patients with $\mathrm{CD}$ showed a significant reduction in the production of PGE2 and IFN- $\gamma$ by circulating monocytes and macrophages ${ }^{(280)}$.

Lung inflammation. Evidence of AA oxidation has been seen in adult respiratory distress syndrome ${ }^{(273)}$. Adults with mild asthma exhibit decreased AA in lung fluid and increased GSSG concentrations which, in the presence of normal plasma concentrations, are suggestive of oxidative stress in the airways ${ }^{(281)}$. Cross-sectional studies showed an inverse relation between plasma vitamin $\mathrm{C}$ and lung inflammation ${ }^{(282)}$ and suggest that high plasma AA concentrations or vitamin C intakes have a positive impact on lung function ${ }^{(283,284)}$. Compared with the highest quintile, serum vitamin $\mathrm{C}$ concentrations in the lowest quintile were associated with an increased risk of asthma in children and adolescents ${ }^{(285)}$. 
Low maternal vitamin $\mathrm{C}$ intake during pregnancy is associated with asthma in 5-year-old children ${ }^{(286)}$. Asthmatic children showed reduced vitamin $\mathrm{C}$ concentrations in leucocytes $(50 \%)$ and plasma $(35 \%)^{(287)}$. Small clinical studies showed that vitamin $\mathrm{C}$ supplements of $2 \mathrm{~g} / \mathrm{d}$ exert protective effects on airway responsiveness to viral infections and allergens ${ }^{(288)}$, but have little effect on exercise-induced asthma ${ }^{(289)}$, although one study did report attenuated exercise-induced bronchoconstriction in patients with asthma ${ }^{(290)}$.

Rheumatoid arthritis. Evidence of AA oxidation has been seen in $\mathrm{RA}^{(272)}$. An intervention study with a Mediterranean diet, rich in antioxidant containing foods, in RA showed a negative correlation between plasma vitamin $\mathrm{C}$ levels and disease activity ${ }^{(291)}$.

CVD. Vitamin $\mathrm{C}$ and other single vitamin supplementation and CVD have been reviewed recently ${ }^{(292)}$. Associations between antioxidants and markers of endothelial function (sICAM-1; flow-mediated vasodilation) and inflammation (CRP, fibrinogen and white blood corpuscles count) were studied in subjects recruited from the general population. Plasma vitamin C and CRP concentrations were inversely related $^{(293)}$. Recent randomised controlled trials have shown that vitamins $\mathrm{C}$ plus $\mathrm{E}$ improved arterial stiffness and endothelial function in patients with essential hypertension ${ }^{(294)}$, vitamin $\mathrm{C}$ decreased sPselectin concentration and vitamins $\mathrm{C}$ plus $\mathrm{E}$ decreased SICAM-1 in patients with chronic degenerative aortic stenosis with or without concomitant coronary artery disease ${ }^{(295)}$.

\section{Vitamin E}

Description, sources, normal intakes and roles. Vitamin $\mathrm{E}$ is an umbrella term for a number of tocopherols and tocotrienols. They are composed of a chromanol ring and a 16-C phytyl tail, which is saturated in the tocopherols and unsaturated in the tocotrienols. The chromanol ring is substituted with one, two or three methyl groups and one hydroxyl group, which exerts the antioxidant function. Depending on the number and positions of the methyl groups of the chromanol ring, $\alpha$ - (three methyl groups at $\mathrm{C}-5, \mathrm{C}-7$ and $\mathrm{C}-8$ positions), $\beta$ - (two methyl groups at $\mathrm{C}-5$ and $\mathrm{C}-8$ positions), $\gamma$ - (two methyl groups, $\mathrm{C}-5$ position unsubstituted) and $\delta$ - (one methyl group at $\mathrm{C}-8$ position) forms can be distinguished for both tocopherols and tocotrienols. These differences in the chemical structure have an impact not only on the hydrophobicity of the different types of tocopherols, but also on the discrimination by the $\alpha$-tocopherol-transfer protein in the liver, which recognises the fully methylated aromatic ring of $\alpha$-tocopherol ${ }^{(296)}$. Consequently, $\alpha$-tocopherol is incorporated into nascent VLDL, while $\gamma$-tocopherol is metabolised and excreted, resulting in substantially lower $\gamma$ - than $\alpha$-tocopherol concentrations in plasma and tissues. The physiological metabolites of tocopherols and tocotrienols, i.e. the carboxyethyl hydroxychromans (CEHC), are generated via a similar degradation pathway that involves cytochrome $\mathrm{P} 450$-mediated $n$-oxidation of the phytyl chain and subsequent $\beta$-chain oxidation to the corresponding hydrophilic metabolites in the absence of modification of the chromanol ring ${ }^{(297,298)}$. The hydroxyl group on the chromanol ring exerts the antioxidant action. It can be esterified to give rise to tocopherol acetate or succinate.

Vitamin E is widely distributed in different kinds of foods. Dietary vitamin E mainly consists of $\alpha$ - and $\gamma$-tocopherol.
Frequently, the major source of vitamin $\mathrm{E}$ is $\gamma$-tocopherol, which is present at high concentrations in plant seeds and vegetable oils such as maize oil and soyabean oil, as well as in nuts such as walnuts, pecans and peanuts ${ }^{(299,300)}$. Fats and oils contributed about $20 \%$, vegetables $15 \%$, meat and fish $12.5 \%$, desserts $10 \%$, breakfast cereals $9 \%$, fruits $5 \%$, bread and grain products $5 \%$, dairy products $4.5 \%$, mixed main dishes $4 \%$, nuts and seeds $4 \%$, eggs $3 \%$, salty snacks $3 \%$, legumes $2 \%$ and soups, sauces and gravies $2 \%$ of the total vitamin $\mathrm{E}$ intake among American adults ${ }^{(301)}$. North American dietary reference intakes for vitamin $\mathrm{E}$ have recently been set at $15 \mathrm{mg} / \mathrm{d}$ for both men and women ${ }^{(258)}$. Vitamin E supplements further contribute to vitamin $\mathrm{E}$ intake. In the Boston Nutritional Status Survey, $38 \%$ of the men of 60 years of age and older and $49 \%$ of the women were users, and $68 \%$ and $73 \%$, respectively, of the male and female users took vitamin E supplements ${ }^{(302)}$. In contrast to vitamin $\mathrm{E}$ in the diet, almost all supplements contain $\alpha$-tocopherol exclusively.

Vitamin $\mathrm{E}$ is a potent chain-breaking antioxidant that acts mainly in the lipid phase and interrupts the chain reaction of lipid peroxidation and, consequently, prevents the propagation of free radical-initiated reactions ${ }^{(303,304)}$. There are differences between $\alpha$ - and $\gamma$-tocopherols ${ }^{(304)}$ and a recent review has paid attention to $\gamma$-tocopherol ${ }^{(305)}$. $\alpha$-Tocopherol decreases lipid peroxidation and enhances LDL resistance to oxidation $^{(306,307)}$. $\gamma$-Tocopherol decreases the concentrations of biomarkers of oxidative stress, including LDL oxidation, MDA formation and superoxide anion generation and is more potent than $\alpha$-tocopherol ${ }^{(308,309)}$. $\alpha$-Tocopherol also decreased the expression of the scavenger receptors SR-A and CD36, which are important in the formation of foam cells in the early stages of atherogenesis ${ }^{(310)}$. However, $\gamma$-tocopherol cannot replace $\alpha$-tocopherol in its functions in fertility $^{(311)}$.

A more recently discovered mechanism of action has been restricted to $\gamma$-tocopherol and its physiological metabolite, $\gamma$-CEHC. Because of an unsubstituted position on the chromanol ring, $\gamma$-tocopherol traps certain reactive nitrogen species in cell lines and unsaturated liposomes with formation of 5-nitro$\gamma$-tocopherol ${ }^{(312,313)}$.

Effects on inflammatory processes and mechanisms of action. One of the mechanisms of action of vitamin $\mathrm{E}$ on inflammatory processes is its well-known antioxidant activity, which limits the generation of ROS as well as the perpetuation and damaging effects of the reactions initiated by ROS, which in turn, could activate the inflammatory process.

$\alpha$-Tocopherol, especially at high doses, decreases the release of pro-inflammatory cytokines, the chemokine IL-8 and PAI-1 and decreases adhesion of monocytes to endothelium ${ }^{(314)}$. Vitamin E (particularly $\gamma$-tocopherol) has been shown to decrease NF- $\kappa$ B activation ${ }^{(315)}$. Additional effects include inhibition of the activities of protein kinase $\mathrm{C}$, 5 -LOX, tyrosine kinase and COX-2 ${ }^{(316,317)}$. $\alpha$-Tocopherol has also been shown to decrease CRP levels ${ }^{(314)}$.

$\gamma$-Tocopherol and $\gamma$-CEHC exert actions not shared by $\alpha$-tocopherol and $\alpha$-CEHC. For instance, $\gamma$-tocopherol but not $\alpha$-tocopherol inhibits PGE2 generation in cell culture ${ }^{(318-320)}$. Furthermore, LTB4, a potent chemotactic agent synthesised by 5-LOX of neutrophils, and TNF- $\alpha$ were decreased in carrageenan-induced inflammation in 
rats and in different cell lines by $\gamma$-tocopherol, but not by $\alpha$-tocopherol ${ }^{(319,320)}$. $\quad \gamma$-Tocopherol decreased oxidised LDL-mediated NF- $\mathrm{B}$ acitivation in human coronary artery endothelial cells ${ }^{(321)}$. $\gamma$-Tocopherol and $\gamma$-CEHC, but not $\alpha$-tocopherol, inhibited COX activity in macrophages and epithelial cells ${ }^{(322)}$.

\section{Effects in inflammatory conditions}

Rheumatoid arthritis. Low antioxidant level (the lowest $v$. higher tertiles of the product of molar concentrations of $\alpha$-tocopherol, $\beta$-carotene and selenium) was a risk factor for RA over 20 years of follow-up of a case-control study ${ }^{(323)}$. In the KRN/NOD mouse, a mouse model of RA, vitamin $\mathrm{E}$ (RRR $\alpha$-tocopherol) prevented joint destruction as assessed by histological analysis, but did not have an effect on clinical features such as time of onset or disease intensity. In addition, levels of IL-1 $\beta$, the main cytokine involved in articular destruction, were reduced, as was neutrophil activation measured by chemiluminescence ${ }^{(324)}$. In an animal model for RA, mice fed a fish oil and additional vitamin E-enriched diet showed significantly lower serum IL-6, TNF- $\alpha$, PGE2, TXB2 and LTB4 levels compared with the fish oil diet alone ${ }^{(325)}$. In a double-blind placebo-controlled trial patients with RA, who received $600 \mathrm{mg}$ vitamin $\mathrm{E}$ twice a day, showed a significant pain reduction but no changes in clinical and biochemical indices of inflammation ${ }^{(326)}$.

$C V D$. Vitamin E inhibits LDL oxidation both when induced by copper ions ex vivo ${ }^{(307,327)}$ and in cell culture ${ }^{(328)}$. In addition to that vitamin $\mathrm{E}$ could have an effect on different aspects of atherogenesis, given that it (i) inhibits smooth muscle cell proliferation by inhibiting protein kinase $C^{(329)}$; (ii) inhibits platelet adhesion, aggregation and release ${ }^{(330)}$; (iii) decreases monocyte adhesion to the endothelium by downregulating the expression of adhesion molecules and reducing monocyte superoxide production ${ }^{(331)}$; (iv) increases prostacyclin synthesis in human endothelial cells, which is a potent vasodilator and inhibitor of platelet aggregation ${ }^{(332,333)}$; (v) mediates upregulation of the expression of cytosolic phospholipase $\mathrm{A} 2$ and $\mathrm{COX}^{(334)}$; and (vi) inhibits the expression of ICAM-1 and VCAM-1 in endothelial cells in culture ${ }^{(335)}$.

While epidemiological studies consistently showed an inverse correlation between vitamin $\mathrm{E}$ status and risk of CVD, the results of vitamin $E$ supplementation trials have been much less convincing regarding prevention of CVD. A possible explanation could be the fact that only $\alpha$-tocopherol, which is almost exclusively present in vitamin E supplements, has been taken in these studies. This is further supported by the observation that $\gamma$-, not $\alpha$-tocopherol serum levels are reduced in $\mathrm{CHD}^{(336)}$. It is known from several intervention trials $^{(337,338)}$ that $\alpha$-tocopherol supplementation causes a marked drop in $\gamma$-tocopherol concentrations in plasma and lipoproteins.

\section{Carotenoids}

Description, sources, normal intakes and roles. Carotenoids represent a group of over 600 coloured pigments found in nature that are responsible for the typical colour of fruits and vegetables as well as some animals. Carotenoids are composed of a 40-carbon atom skeleton and are either hydrocarbons (also called xanthophylls) that contain only $\mathrm{H}$ and $\mathrm{C}$ ( $\alpha$-, $\beta$-carotene, lycopene), or oycarotenoids that contain also $O$ (lutein, zeaxanthin). Provitamin A carotenoids ( $\alpha$ and $\beta$-carotene, and $\beta$-cryptoxanthin) are carotenoids that can be cleaved in the intestine either by central cleavage, giving rise to two molecules of retinol from one molecule $\beta$-carotene, or excentric cleavage, giving rise to one molecule of retinol and carotenoid cleavage products.

Plasma carotenoid concentrations are useful biomarkers of the consumption of fruits and vegetables ${ }^{(339)}$. The most prevalent carotenoids in the Western diet are $\alpha$-carotene, $\beta$-carotene, lycopene, $\beta$-cryptoxanthin, lutein and zeaxanthin. Dietary sources of $\alpha$-carotene are carrots, sweet potatoes, cabbage, spinach, pumpkin, broccoli and pepper; of $\beta$-carotene are carrots, pumpkin, red pepper, green beans, green salad, broccoli and melon; of $\beta$-cryptoxanthin are avocado, green olives, peach, mandarin, mango, papaya and orange; of lycopene are tomatoes and tomato products, and guave; of lutein are dark green leafy vegetables such as cabbage, spinach, broccoli, peas, cauliflower and green salad; of zeaxanthin is maize. Most of the naturally occuring carotenoids are present as all-trans isomers and are transformed into the cis isomers during food processing (heating; lycopene) or in the intestinal mucosa cells ( $\beta$-carotene). Astaxanthin is found in marine animals and vegetables.

As suggested by Krinsky ${ }^{(340)}$, the biological activities of carotenoids should be diffentiated as:

(i) functions, including acting as accessory pigments in photosynthesis, protection against photosensitisation and role as provitamin $\mathrm{A}$;

(ii) actions, including antioxidant, immunoenhancing, antimutagenic and anti-carcinogenic actions;

(iii) associations, including decreasing risk of macular degeneration and cataracts, some cancers and some cardiovascular events.

Even though carotenoids are recognised mainly for their provitamin A functions, less than $10 \%$ of them exert provitamin $\mathrm{A}$ activities in mammals. Of the carotenoids, $\beta$ carotene has the highest provitamin A activity. The efficiency of $\beta$-carotene conversion to vitamin A decreases with higher $\beta$-carotene intake. Lutein is among the most prevalent carotenoids found in normal skin and a major carotenoid in the macular pigment and effectively filters blue light and thus protects the retina from light-induced oxidative damage $^{(341)}$.

Effects on inflammatory processes and mechanisms of action. Carotenoids possess immunomodulatory activities in human subjects and animals, including, for instance, stimulation of the phagocytic and bacteria-killing ability of peripheral blood neutrophils and peritoneal macrophages and of lymphocyte blastogenesis, increasing the population of specific lymphocyte subsets and lymphocyte cytotoxic activity, as well as stimulation of the production of various cytokines. $\beta$-Carotene inhibits inflammatory gene expression by suppressing the activation of the redox-sensitive transcription factor, NF- $\mathrm{KB}^{(342,343)}$.

Effects in inflammatory conditions. While $\beta$-carotene has been studied quite extensively, data are limited for other carotenoids. In Table 2, the effects of carotenoids as well as of vitamins $\mathrm{C}$ and $\mathrm{E}$ are listed. Biomarkers of inflammation have been included in a variety of studies and quite 
Table 2. Summary of studies investigating the anti-inflammatory actions of vitamins $C$ and $E$ and of carotenoids

\begin{tabular}{|c|c|c|c|}
\hline Compounds & Model/study subjects & Effects & Reference \\
\hline \multicolumn{4}{|l|}{ Cell culture studies } \\
\hline$\gamma$-Tocopherol, $\gamma$-CEHC & Macrophages, epithelial cells & $\downarrow$ PGE2, LTB4, TNF- $\alpha$, COX activity & 319,322 \\
\hline Lycopene & Dendritic cells & $\downarrow$ NF-кB, IL-2, IL-12, ERK 1/2, p38, JNK & 348 \\
\hline \multicolumn{4}{|l|}{ Animal studies } \\
\hline Vitamin E & Transgenic mouse model of RA, fish oil treated & $\downarrow$ Serum IL-6, IL-10, IL-12, TNF- $\alpha$ & 325 \\
\hline$R R R \alpha$-tocopherol & Transgenic mouse model of RA & Prevention of joint destruction, $\downarrow$ IL- $1 \beta$ & 324 \\
\hline$\beta$-Carotene & Mice, LPS-treated & $\downarrow$ NO, PGE2, iNOS, COX-2, TNF- $\alpha$, IL-1 $\beta$, NF-кB, iNOS promoter activity & 342 \\
\hline Lycopene & Rats, induced colitis & $\downarrow$ Colonic thickness, area of inflammation, myeloperoxidase & 354 \\
\hline Lutein & Mice, UV irradiation & $\downarrow$ Ear swelling & 350 \\
\hline Astaxanthin & Rats, induced uveitis & $\downarrow$ PGE2, TNF- $\alpha, N O$, iNOS enzyme activity & 349 \\
\hline \multicolumn{4}{|c|}{ Observational studies in human subjects } \\
\hline Vitamin E & Pregnancy, children with asthma & $\downarrow$ Maternal vitamin $E$ intake associated with $\uparrow$ asthma and wheezing in children & 286 \\
\hline \multicolumn{4}{|c|}{ Association studies in human subjects } \\
\hline$\beta$-Carotene & Patients with RA & $\downarrow$ Serum $\beta$-carotene preceding diagnosis & 358 \\
\hline Different carotenoids & General population & $\begin{array}{l}\uparrow \text { Plasma lutein, lycopene associated with } \downarrow \text { sICAM-1; } \\
\uparrow \beta \text {-carotene associated with } \downarrow \text { CRP, leucocytes; } \\
\uparrow \text { zeaxanthin: } \downarrow \text { flow-mediated vasodilation }\end{array}$ & 293 \\
\hline Different carotenoids & Critically ill patients & $\uparrow$ Carotenoids associated with $\downarrow$ CRP & 346 \\
\hline Different carotenoids & NHANES III, non-smokers & $\begin{array}{l}\uparrow \text { Plasma carotenoids (all) associated with } \downarrow \text { CRP; } \\
\uparrow \beta \text {-cryptoxanthin associated with } \downarrow \text { fibrinogen; } \\
\uparrow \beta \text {-carotene, lycopene associated with } \downarrow \text { WBC }\end{array}$ & 345 \\
\hline Different carotenoids & Abdominal obesity & $\downarrow$ Serum canthaxanthin, $\beta$-carotene, $\alpha$-carotene associated with $\uparrow$ waist and waist:hip ratio & 361 \\
\hline \multicolumn{4}{|c|}{ Case-control studies in human subjects } \\
\hline Vitamin C, $\alpha$-carotene & NHANES III, asthmatic children & $\downarrow$ serum vitamin $\mathrm{C}, \alpha$-carotene associated with $\uparrow$ risk of asthma & 285 \\
\hline $\begin{array}{l}\text { Vitamin } E, \beta \text {-carotene } \\
(+ \text { selenium })\end{array}$ & Control group for cancer study & $\downarrow$ Antioxidant index associated with $\uparrow$ risk of RA & 323 \\
\hline Lycopene & Women's Health Study & $\uparrow$ Plasma lycopene associated with $\downarrow$ risk of CVD & 359 \\
\hline Different carotenoids & EPIC Study & $\uparrow \beta$-Cryptoxanthin or $\uparrow$ zeaxanthin intake associated with $\downarrow$ risk of inflammatory polyarthritis & 357 \\
\hline \multicolumn{4}{|c|}{ Intervention studies in human subjects } \\
\hline$\alpha$-tocopherol & Patients with RA & $\downarrow$ Pain & 326 \\
\hline Vitamin $\mathrm{C}+\alpha$-tocopherol & Patients with hypertension & Flow-mediated dilation, central pulse wave velocity & 294 \\
\hline Vitamin $\mathrm{C}+\alpha$-tocopherol & Patients with IBD & $\downarrow$ Oxidative stress & 279 \\
\hline $\begin{array}{l}\text { Carotenoid-rich fruits \& } \\
\text { vegetables }\end{array}$ & Healthy non-smoking men & $\downarrow \mathrm{CRP}$ & 351 \\
\hline Lycopene & Healthy young volunteers & $\downarrow$ TNF- $\alpha$ production & 352 \\
\hline $\begin{array}{l}\text { Vitamin } A, C, E+\text { selenium } \\
+ \text { fish oil }\end{array}$ & Patients with IBD & $\downarrow$ PGE2, IFN- $\gamma$ & 280 \\
\hline Mediterranean diet & Patients with RA & $\uparrow$ plasma vitamin $\mathrm{C}$ associated with $\downarrow$ disease activity score & 290 \\
\hline
\end{tabular}

LT, leukotriene; COX, cyclo-oxygenase; CEHC, carboxyethyl hydroxychroman; ERK, extracellular signal-regulated kinase; JNK, jun N-terminal kinase; iNOS, inducible NO synthase; RA, rheumatoid arthritis; ICAM, intercellular adhesion molecule; CRP, C-reactive protein; WBC, white blood cell count; NHANES, National Health and Nutrition Examination Survey; EPIC, European Prospective Investigation of Cancer; IBD, inflammatory bowel disease; IFN, interferon: MDA, malondialdehyde. 
consistently either showed an inverse relation between status of different carotenoids and inflammation or reduction in elevated biomarkers in response to intervention using carotenoidrich foods or supplements. Clinical end point studies are particularly scarce. Like other serum antioxidants such as vitamin $\mathrm{C}$, plasma carotenoid concentrations decline during the acute-phase response to infection and injury in the presence of increased levels of biomarkers of inflammation ${ }^{(344)}$. Inverse relations between plasma $\beta$-carotene concentrations and biomarkers of inflammation have been shown in a re-evaluation study of the National Health and Nutrition Examination Survey III study ${ }^{(345)}$, as well as in studies in critically ill patients ${ }^{(346)}$. The underlying mechanisms are not known, but may involve a cellular carotenoid-binding protein ${ }^{(347)}$. Most importantly, however, $\beta$-carotene and retinol levels are restored after resolution of the inflammatory state in the absence of $\beta$-carotene and/or retinol supplements. Study of the effects of lycopene on murine bone marrow-derived DC, which are the most potent antigen-presenting cells, revealed that lycopene downregulates NF- $\mathrm{KB}$ as well as mitogenactivated kinases such as extracellular signal-regulated kinase $1 / 2$, p38 and jun N-terminal kinase ${ }^{(348)}$. Astaxanthin showed a dose-dependent anti-inflammatory effect in endotoxin-induced uveitis in rats by suppressing NO, PGE2 and TNF- $\alpha$ production ${ }^{(349)}$. Mice fed lycopene showed significant inhibition of ear swelling induced by UV radiation ${ }^{(350)}$. In a RCT, high intake of carotenoid-rich fruits and vegetables reduced CRP concentrations ${ }^{(351)}$. In another RCT, dietary intake of a tomato-based drink (providing $5.7 \mathrm{mg}$ lycopene, $1 \mathrm{mg} \beta$-carotene, $3.7 \mathrm{mg}$ phytoene and $2.7 \mathrm{mg}$ phytofluene and $1.8 \mathrm{mg} \alpha$-tocopherol per day), was associated with $34 \%$ decrease in TNF- $\alpha$ in whole blood ${ }^{(352)}$

Inflammatory bowel diseases. Plasma concentrations of different carotenoids are decreased in patients with $\mathrm{CD}^{(277,353)}$. In a rat model of colitis, lycopene efficiently counteracted the inflammatory response and mucosal damage in iron-supplemented animals ${ }^{(354)}$, which can be explained by the fact that transition metal iron can give rise to the formation of hydroxyl radical, the most reactive and cytotoxic ROS that has the capacity of oxidising biomolecules including PUFA, proteins and DNA bases and that lycopene acts as an antioxidant.

Asthma. Whole blood concentrations of total carotenoids and lycopene were decreased in patients with asthma compared with controls and correlated with sputum concentrations of carotenoids ${ }^{(355)}$.

Rheumatoid arthritis. An inverse association between serum $\beta$-cryptoxanthin levels and RA has been reported in women aged 55-69 years ${ }^{(356)}$. A nested case-control study within the European Prospective Investigation of Cancer Incidence study focused on dietary intake and occurrence of new cases of inflammatory polyarthritis and showed that subjects with dietary intake of zeaxanthin and $\beta$-cryptoxanthin in the highest tertile were at lower risk of developing inflammatory polyarthritis compared with those in the lowest tertile. The effect of $\beta$-cryptoxanthin remained significant also after adjustments for total energy and protein intakes as well as for smoking ${ }^{(357)}$. In another study, plasma $\beta$-carotene concentrations preceding diagnosis of RA by $2-15$ years were lower in patients who developed RA compared with controls ${ }^{(358)}$.
$C V D$. Association between antioxidants and markers of endothelial function (sICAM-1 and flow-mediated vasodilation) and inflammation (CRP, fibrinogen and white blood cell count) were studied in subjects recruited from the general population. Lutein and lycopene were inversely related to sICAM-1 and $\beta$-carotene was inversely related to white blood cell count and $\mathrm{CRP}^{(293)}$. In a nested case-control study within the Women's Health Study, CVD risk was associated with plasma lycopene concentrations such that women in the upper three quartiles of plasma lycopene concentrations had a $50 \%$ risk reduction ${ }^{(359)}$. Similar results were not observed in men participating in the Physicians' Health Study ${ }^{(360)}$.

Obesity. Low carotenoid and elevated CRP concentrations are frequently observed in obesity, and are attributed to unfavourable dietary habits and low-grade inflammation $^{(361,362)}$. An inverse correlation between plasma carotenoid concentrations and BMI was reported in obese children and adolescents ${ }^{(363)}$.

\section{Flavonoids}

Description, sources, normal intakes and roles. Polyphenols are secondary metabolites of plants involved in pigmentation, reproduction and protection against pathogens ${ }^{(364)}$. Presently there are more than 8000 known polyphenolic substances sharing a common chemical structure (hydroxyl group on aromatic ring) with different constituents. Flavonoids are the most abundant polyphenols present in the human diet and represents a subclass of molecules characterised with a C6-C3-C6 backbone structure ${ }^{(364)}$. Flavonoids can be divided into several classes according to different constituents such as flavanones, flavone, flavanols and flavonols. They can be found in almost all plant foods and, among the flavonols, myricetin, kaempferol and quercetin are the most representative, while catechins are the most abundant flavanols contained in tea leaves. Flavanones are mainly represented in the diet by taxifolin, naringinin and hesperitin. The main sources of flavanones are citrus fruits. Flavones, luteolin, wogonin and apigenin, are less common and identified in sweet red pepper and celery. In addition to these, other classes of flavonoids are present in the diet such as proanthocyanidins and their oligomers.

Dietary intake of flavonoids, calculated in 1976 in the $\mathrm{US}^{(365)}$, was $1 \mathrm{~g} / \mathrm{d}$ with $16 \%$ as flavonols, flavones and flavanones; $20 \%$ as catechins; $17 \%$ as anthocyanins and $45 \%$ as bisflavones. However, the high variability of polyphenol intake, the lack of homogeneous database and reliable biomarkers of exposure make it difficult to provide a reliable estimate of flavonoid intake in the different countries.

Over the last years, there have been significative advances in the understanding of the absorption and metabolism of flavonoids. In order to be absorbed, flavonoid glycosides, the most abundant form in food, are hydrolysed by intestinal enzymes or colon microflora. During the process of absorption, flavonoids are conjugated in the small intestine and later in the liver to increase their hydrophilicity and facilitate their elimination from the body in urine and bile. The circulating forms of flavonoids are mainly conjugated and evidence regarding their accumulation in specific target tissues is still lacking.

Flavonoids have been suggested to have anti-inflammatory activity through several action mechanisms involving the 
reduction in the concentration of prostanoids and leukotrienes through the inhibition of eicosanoid generating enzymes such as phospholipase A2, COX and $\operatorname{LOX}^{(366)}$.

Anti-inflammatory actions of flavonoids. Quercetin was the first flavonoid shown to inhibit neutrophil phospholipase $\mathrm{A} 2^{(367)}$ with an IC50 of $57-100 \mu \mathrm{M}$. Flavanones such as hesperitin and naringenin were shown to be less effective inhibitors of snake venom phospholipase A2 compared with the flavonols quercetin, kaempferol and myricetin ${ }^{(368)}$. Biflavonoids such as amentoflavone, ginkgetin, ochnaflavone and iso-ginkgetin were shown to be good inhibitors, with an IC50 of about $10 \mu \mathrm{M}$ for phospholipase A2 from several sources. Luteolin, 3,4-dihydroxy-flavone, galangin, apigenin and morin were the first flavonoids to be shown to inhibit $\mathrm{COX}^{(366)}$. Quercetin and kaempferol are potent inhibitors of COX from rat peritoneal macrophages ${ }^{(368)}$. The flavones apigenin, luteolin, galangin, kaempferol and quercetin and the biflavonoids amentoflavone, broussochalcone A and kuraridin are potent COX-1 inhibitors, although with different potencies. Catechin weakly inhibited COX-2 but at very high concentration $(100 \mu \mathrm{M})^{(369)}$. Flavonols such as kaemferol, quercetin, morin and myricetin were found to be better LOX inhibitors than flavones and with a preferential effect on 5-LOX compared with 12-LOX. Flavanones such as naringenin were not inhibitory against 5- and 12-LOX. Endothelial NOS was inhibited weakly by quercetin at very high concentration (IC50 $220 \mu \mathrm{M})^{(370)}$ with a lack of effect on nNOS and iNOS. Several flavonoids (rutin, hepseridin, catechin and tricin) had no effect on the three forms of NOS. Using LPSor cytokine-treated macrophages or macrophage cell lines for stimulated NO production, quercetin, apigenin and luteolin were found to inhibit NO production ${ }^{(371)}$, downregulating iNOS expression. Catechins and flavanones at concentrations below $100 \mu \mathrm{M}$ did not affect NO production by LPS-treated RAW 264.7 cells $^{(372)}$. Glycosylated flavonoids, such as vitexin, had little effect on NO production with no effect at a concentration of $100 \mu \mathrm{M}$. Flavones such as apigenin, wogonin and luteolin were the most efficient inhibitors (IC50 $10-20 \mu \mathrm{M})$. Furthermore, studies ${ }^{(373)}$ showed that the inhibitory effect of apigenin, genistein and kaempferol on NO production is mediated by an effect on induction of iNOS expression. Taken together, these results suggest that some flavonoids are natural inhibitors of iNOS induction, but not inhibitors of iNOS activity. With regard to inflammatory cytokines, genistein was reported to inhibit IL- $1 \beta$, IL- 6 and TNF- $\alpha$ production by LPS-stimulated human blood monocytes ${ }^{(374)}$. Genistein and silybin were shown to inhibit TNF- $\alpha$ production from LPS-treated RAW cells ${ }^{(375)}$. Flavonoids appear to inhibit the expression of inflammation-related enzymes/proteins partly by suppressing activation of $\mathrm{NF}-\kappa \mathrm{B}$ and $\mathrm{AP}-1$, an effect potentially mediated by the inhibition of different protein kinases (e.g. mitogen-activated protein kinase; extracellular signal-regulated kinase $1 / 2$ ) involved in signal transduction pathway.

Quercetin has been shown to affect iNOS expression by inhibiting p38 MAPK and TNF- $\alpha$ from LPS-induced RAW cells by inhibiting jun N-terminal kinase/SAPK and Ap-1 DNA binding ${ }^{(376,377)}$. TNF- $\alpha$ was also inhibited through inhibition of extracellular signal-regulated kinase $1 / 2$ and p38 MAPK. Quercetin was shown also to affect NF- $\mathrm{B}$ activation by extracellular signal-regulated kinase and p38 kinase inhibition $^{(378)}$. The effect on NF-кB was shown also for genistein, apigenin, kaempferol and epigallocatechin 3-gallate. The evidence so far shows that flavonoids are able to inhibit the expression of inflammation-related enzymes/proteins partly by suppressing activation of NF- $\mathrm{B}$ and AP-1, an effect potentially mediated by the inhibition of different protein kinases involved in the signal transduction pathway.

Regarding the effect of flavonoids on the expression of inflammatory markers and processes in vivo, scientific evidence is largely lacking. Quercetin inhibited release of TNF$\alpha$ from carrageenan-induced air-pouch exudates in rats ${ }^{(379)}$. Quercetin and rutin were found to suppress lethal endotoxic shock induced by LPS in mice ${ }^{(380)}$. Orally administered luteolin was showed to inhibit TNF- $\alpha$ production in LPS-treated mice ${ }^{(381)}$.

Human studies investigating the effect of flavonoids on markers of inflammation are scarce and most of them focus on the use of flavonoid-rich foods and not on pure molecules. One month of supplementation with $30 \mathrm{~g} / \mathrm{d}$ ethanol as wine or as gin in healthy men was able to decrease plasma levels of IL- $1 \alpha$ and fibrinogen ${ }^{(383)}$. However, expression of the several adhesion molecules on monocytes and T-lymphocytes was significantly decreased only after wine ingestion. Wine also decreased serum concentrations of sVCAM-1, sICAM-1 and CRP. In another study ${ }^{(383)}$, supplementation with a grape polyphenol extract containing anthocyanins, quercetin, myricetin, kaempferol and resveratrol (36 g/d for 4 week) produced a significant decrease in plasma TNF- $\alpha$ with no effect on CRP and IL-6 concentrations in both pre- and post-menopausal women. In both studies described earlier, the lack of measurement of polyphenol levels in body fluids does not allow any conclusion about the molecules responsible for the anti-inflammatory effect to be made. In an intervention study where plasma levels of flavonoids were measured, daily black tea supplementation $(900 \mathrm{ml})$ for 4 weeks significantly increased plasma catechin levels $(29 \%)$ compared with baseline values but without any changes on CRP and $\mathrm{TAC}^{(384)}$. Moreover, in a prospective study in non-diabetic women, the intake of flavonols and flavones was not significantly associated to plasma concentrations of CRP and IL-6 or with the development of type 2 diabetes ${ }^{(385)}$. Recently, in the attempt to investigate diet-induced postprandial oxidative and inflammatory stress, acute ingestion of $50 \mathrm{ml}$ of extra virgin olive oil has been shown to decrease plasma levels of inflammatory molecules (LTB4 and TXB2) together with an increase in serum TAC $^{(386)}$.

As overall, only about $1 \%$ of the polyphenol that is consumed is absorbed, with large differences according to the different categories, the plasma concentration that is achieved after eating polyphenol rich food is about $1 \mu \mathrm{M}^{(364)}$. The low concentration of flavonoids in biological fluids might present an obstacle in exerting the putative anti-inflammatory action in vivo. Human studies involving food items need to be linked with a chemical characterisation of the flavonoid composition of the food and with the assessment of specific biomarkers of single phenolics and antioxidant activity. Moreover, information obtained on the basis of acute ingestion studies might be extremely valuable for designing long-term intervention trials. On the basis of the existing evidence, a clear conclusion cannot be drawn and further human trials are needed to elucidate the role of flavonoids as antiinflammatory agents in vivo. 


\section{The gut microflora and inflammation}

Gut microflora. The intestinal epithelium of the gastrointestinal tract and its associated microflora are vital to the protection of the body ${ }^{(387,388)}$. At birth, the gastrointestinal tracts of babies are sterile but then rapidly become colonised by microorganisms from the mother's faecal and vaginal microbiota and the immediate environment. Consumption of oxygen within the gut by the first colonising organisms, facultative anaerobic bacteria, enables fully anaerobic bacteria to then colonise the lower gut. Bifidobacteria are more predominant in breast-fed babies, whereas a more complex population (similar to that of adults) develops in formula-fed babies, which comprises clostridia, bacteroides, enterobacteria, streptococci and a lower level of bifidobacteria ${ }^{(389)}$. SCFA produced by bifidobacteria help to protect the baby against infection. During the first year of life, the intestinal microbiota stabilises and gradually resembles that of an adult. Up to 1014 micro-organisms are contained in the adult human body - ten times more microbial cells than human - most of these microorganisms are located in the gastrointestinal tract ${ }^{(390)}$. More than $1 \mathrm{~kg}$ of bacteria is present in the gut and faeces typically comprise $50 \%$ bacteria, meaning that human subjects excrete $50-100 \mathrm{~g}$ of bacteria each day. Micro-organisms are at their lowest numbers $(<103 \mathrm{CFU} / \mathrm{g})$ in the stomach due to its low $\mathrm{pH}$ and fast transit time but reach levels as high as $1012 \mathrm{CFU} / \mathrm{g}$ in the colon, due to its much slower transit time, less acidic $\mathrm{pH}$ and low oxygen levels. Faecal isolates are generally representative of the microbiota of the distal colon. At least 500 different bacterial species have been cultured from faeces (including lactobacilli and bifidobacteria), yet just forty species account for $99 \%$ of those that have been identified. The microbial inhabitants of the intestinal microbiota are still not fully identified due to the limitations of identification techniques and individual variations. New molecular typing methods are now enabling identification of further gut-associated species.

Studies with germ-free animals have demonstrated the importance of the gut microflora for the normal development of the gastrointestinal tract and the education and stimulation of the immune system ${ }^{(391-393)}$. In the absence of an intestinal microflora, the intestinal immune system is underdeveloped and certain functional parameters, such as macrophage phagocytic ability and Ig production, are reduced ${ }^{(394)}$. The intestinal microflora may also be important in protection against atopic disease $^{(395-397)}$, as demonstrated by Sudo et al. ${ }^{(398)}$ who showed that oral tolerance could be restored in germ-free mice by reconstitution of the intestinal microflora with bifidobacteria at the neonatal stage. The intestinal microflora of infants with atopic disease may differ from healthy infants, with higher levels of clostridia and lower levels of bididobacteria in those with atopy ${ }^{(396)}$.

The major function of the colonic microflora is the fermentation of dietary substances that have passed undigested through the small intestine and mucus produced within the gut. Saccharolytic fermentation produces SCFA such as acetate, propionate, butyrate, which are essential nutrients for the colonocytes. The acids have an additional protective benefit by lowering the $\mathrm{pH}$ of the intestinal lumen and by their antimicrobial nature. Butyric acid (produced by bifidobacteria) is particularly important because it is the preferred energy source of the epithelial cells in the colon and influences cell proliferation. It is thought to play a prominent role in reducing the risk of colon cancer. By contrast, some of the end products of proteolytic fermentation are carcinogenic $^{(399)}$. A healthy, or balanced, intestinal microflora is thought to be one that is predominantly saccharolytic and that comprises significant numbers of bifidobacteria and lactobacilli, such that their activities predominate over those that may be harmful (e.g. clostridia, Staphylococcus aureus, toxic E. coli, sulphate-reducers and certain Bacteroides species $)^{(10,400-402)}$. Predominance of the latter groups of bacteria may predispose the host to a number of clinical disorders while increasing susceptibility to infections by transient enteropathogens ${ }^{(402)}$.

\section{Prebiotics}

Description, sources, intakes and roles. The earliest definition of a prebiotic was 'a non-digestible food ingredient that beneficially affects the host by selectively stimulating the growth and/or activity of one or a limited number of bacteria in the colon, and thus improves host health' ${ }^{, 400)}$. As research has progressed, this definition has been refined. There are now three accepted criteria for classifying a food ingredient as a prebiotic.

(i) It should be non-digestible and non-absorbable - it should be resistant to gastric acidity, hydrolysis by intestinal brush border/pancreatic digestive enzymes and gastrointestinal absorption. This makes 'prebiotics' completely available for intestinal microflora.

(ii) It should be selectively fermented by the intestinal (mainly colonic) microflora, meaning that the growth of some groups of bacteria is promoted (such as members of the genus Bifidobacterium and Lactobacillus to which most probiotics belong), others are not affected (commensals such as Bacteroides, Fusarium) and still others are suppressed (pathogenic organisms such as Clostridium perfringens group, Salmonella, coliforms, etc.). This selective interaction with intestinal fermentation also results in additional production of bacterial metabolites (mainly SCFA such as acetate, propionate and butyrate) that are absorbed in the blood of the host and that interact in various ways (e.g. cell signalling function, energy substrate for certain cell types) with the host metabolism.

(iii) It should result in improvement of health-related markers, this being a direct consequence of the selective interaction of prebiotics with intestinal fermentation.

Selective prebiotic interaction with the colonic bacterial ecosystem represents a potentially important improvement of the 'external environment' of the host. This observation is at the basis of prebiotic action: prebiotics selectively interact with resident intestinal microflora and thereby stimulate the development and metabolic activity of organisms that belong to bacterial genera to which the most common 'probiotics' also belong. Moreover, while being fermented, prebiotics themselves are converted into biologically active molecules that pass into the systemic environment. These metabolites may be responsible for at least part of nutritional benefits observed upon eating prebiotics. 
Inulin-type fructans are $\beta(2-1)$-linked fructose oligomers with chain length ranging between three and hundred depending on the plant source ${ }^{(403)}$. These complex carbohydrates are present in most plants that occur in regions with a moderately cold climate, characterised by periods of frost. As a consequence, they are present not only in various foodplants such as wheat, onions, garlic, chicory root, Jerusalem artichoke, etc., but also in fruits such as banana and tomato ${ }^{(404)}$. It has been estimated that via the consumption of food plants, people consume between 1 and $4 \mathrm{~g}$ of inulin per day. At present, inulin (with average chain length of ten) and its partial hydrolysate oligofructose (average chain length of four) are produced on a commercial scale via extraction from the chicory root. The latter is also enzymatically synthesised from sucrose. These products are used globally and are applied in thousands of prepared food products such as dairy, meat products, bakery, drinks, spreads, etc.

Galacto-oligosaccharides are enzymatically synthesised from lactose mainly with transferase enzymes. The link between the galactose moieties depends on the enzyme system used. Galacto-oligosaccharides formed this way are always short-chain molecules, with an average chain length of three. They do not occur as such in nature, although more complex but structurally related forms are present in mammalian breast milk. Combined with long-chain inulin fractions in milk formulations, they were shown to shift the composition of the intestinal microflora of babies close to that seen in breast-fed babies, and at the same time to induce some immunological and anti-inflammatory effects $^{(405)}$. There also is evidence that lactulose has prebiotic properties. However, lactulose induces osmotic effects in the intestine and acts as a laxative; consequently, it is not considered a useful food ingredient. There are various other candidate prebiotics for which evidence of prebiotic properties, and certainly of potential health benefits, needs to be demonstrated (isomalt, xylo-oligosaccharides, lactosucrose, soyabean oligosaccharides, isomalto-oligosaccharides, etc.).

Prebiotics and experimental models of inflammation. Inulin increased colonic lactobacilli and decreased cecal $\mathrm{pH}$ in rats subjected to DSS-induced intestinal inflammation ${ }^{(406)}$. Inulin decreased the lesion score of the colonic mucosa, which correlated very well with the reduction in intraluminal release of inflammatory markers such as PGE2, TXB2 and LTB4 and reduction in mucosal myeloperoxidase activity, a marker of neutrophil accumulation within the tissue.

Schultz et al. ${ }^{(407)}$ used two groups of HLA-B27 transgenic rats that spontaneously develop immune-mediated colitis and administered both a prebiotic (oligofructose) and probiotics (L. acidophilus and Bifidobacterium lactis). They observed a significant improvement of the histologic score, which was correlated to prebiotic effects on the ceco-colonic microflora. No probiotic bacterial strains were detected in the faeces. In a similar model, Hoentjen et al. ${ }^{(408)}$ administered a combination of rapidly and slowly fermented fructoligosaccharides and observed significant improvement of histologic score in the cecum and colon. The authors observed a concomitant improvement of inflammation markers such as a decrease in IL-1 $\beta$ and an increase in TGF- $\beta$ in the cecal tissue, and a decrease in IFN- $\gamma$ in the mesenteric lymph nodes. These effects again correlated with the impact of the dietary prebiotics on cecal microflora (increased lactobacilli and bifidobacteria), implying that the selective prebiotic interaction with the intestinal flora was the basis of the observations. A long-term (33 week) study of tumour formation and immune function in AOM-treated rats revealed that prebiotics (inulin), and a combination of prebiotics and probiotics, had different effects on the gut-associated lymphoid tissue than probiotics (Lactobacillus rhamnosus and B. lactis) ${ }^{(409)}$.

Prebiotics and inflammatory conditions. Saavedra et al. ${ }^{(410)}$ showed reduced absenteeism, visits to the physician and fever-associated diarrhoea in kindergarten-aged children given prebiotics. These results have been recently confirmed by Waligora-Dupriet ${ }^{(411)}$ in 6- to 24-month-old infants who were given a rapidly fermented prebiotic (oligofructose). Reduced flatulence and diarrhoea, as well as vomiting and fever - clinical observable consequences of inflammation were correlated with the typical selective prebiotic modification of the intestinal flora (increased bifidobacteria and decreased C. perfringens and Staphylococcus spp.). In another study, a combination of galacto-oligosaccharides and long-chain inulin decreased the incidence of atopic dermatitis in babies at high risk $^{(412)}$. This effect was accompanied by a significant modification of the composition of the intestinal microflora, characterised by higher Bifidobacterium spp. counts.

The anti-inflammatory effects of prebiotics have been investigated in patients suffering from inflammatory conditions. Welters et al. ${ }^{(413)}$ administered prebiotic chicory inulin to familial adenomatous polyposis patients with ileal pouchanal anastomosis. There was a significant decrease in the pouchitis disease activity index, confirmed by a decrease in endoscopic score, which correlated with a modified faecal short-chain fatty acid pattern (increased butyrate), which is a direct consequence of selective interaction of the administered prebiotics on intestinal flora. In an exploratory study, Casellas et al. ${ }^{(414)}$ administered either oligofructose-enriched inulin or placebo (maltodextrin) to patients with active UC and on standard drug therapy (mesalazine). The prebiotic induced a faster reduction in faecal calprotectin levels than that seen in the control group. However, the rate of clinical remission was similar in both placebo and prebiotic groups, as all patients were on standard medical therapy with mesalazine. Furrie et al. (27) administered a synbiotic composed of oligofructose-enriched inulin as a prebiotic and Bifidobacterium longum as a probiotic to patients with UC. Sigmoidoscopy scores were reduced in the synbiotic group as compared with control. Mucosal mRNA levels for human $\beta$-defensins (hBD2, hBD3 and hBD4) that are upregulated in active UC were reduced in the synbiotic group as were inflammatory cytokines such as TNF- $\alpha$ and IL1- $\alpha$. Biopsies in the synbiotic group had reduced inflammation and epithelial tissue was regenerated. These anti-inflammatory effects of the synbiotic combination were accompanied by a typical prebiotic-type shift in composition of the intestinal microflora, characterised by a 42-fold increase in numbers of Bifidobacterium spp. associated with the mucosa.

\section{Probiotics}

Description, sources, normal intakes and roles. The FAO of the UN (FAO) and the WHO have recognised the potential of probiotic foods to provide health benefits and the use of specific strains as safe supplements for human consumption. Probiotics are defined as 'live micro-organisms, which, when 
administered in sufficient amounts, confer a health benefit to the host ${ }^{\prime(415)}$. Lactic acid-producing bacteria, including lactobacilli and bifidobacteria from human origin, are the most commonly used probiotics. Foods containing probiotic bacteria range from dairy fermented milk products to probiotic powders, drinks or supplements in a lyophilised form. Probiotic effects can be direct or indirect through modulation of the endogenous intestinal microflora or immune system. Their fate in the gastrointestinal tract and their effects differ among strains. For most of the strains, the effective dose is between $10^{8}$ and $10^{10} \mathrm{cfu} / \mathrm{d}$. Over the past decade, progress has been made to provide scientifically and clinically valid evidence on the impact of defined probiotic strains on human health.

Mechanisms of anti-inflammatory action. Experimental models have revealed that probiotics differ greatly in their mechanism of action, including modification of the local microflora, epithelial barrier function, inflammation or the immune system. Several probiotic bacteria adhere to mucosal tissue in a strain-dependent manner, and by this transiently enhance intestinal persistence and limit pathogen access to the epithelium ${ }^{(416,417)}$. Modulation of the gastrointestinal microenvironment and inhibition of pathogen growth by probiotics was demonstrated via the release of antimicrobial factors, such as defensins, bacteriocins, hydrogen peroxide, nitric oxide, and SCFA and reduction in the luminal $\mathrm{pH}^{(418)}$. Several probiotics, such as L. acidophilus ATCC4356 or VSL\#3, a mixture of four different lactobacilli, three bifidobacteria and Streptococcus thermophilus, have been shown to successfully preserve epithelial barrier function by induction of mucin secretion, maintenance of cytoskeletal integrity, tight junction protein phosphorylation or induction of heat-shock proteins ${ }^{(419,420)}$. The anti-inflammatory effect of a number of probiotic bacteria, including lactobacilli and bifidobacteria, seems to be based on their properties to directly interact with intestinal epithelial cells, which have a key role in sensing danger signals within the luminal microenvironment. Some probiotic bacteria antagonise NF- $\kappa \mathrm{B}$ activation by degradation of IкB- $\alpha$, or regulating the nuclear export of $\mathrm{NF}-\kappa \mathrm{B}$ relA or NF- $\mathrm{B}$ p65 subunits in a PPAR- $\gamma$-dependent manner ${ }^{(421,422)}$. Also, expression of pro-inflammatory genes, such as TNF- $\alpha$, IL- 1 or neutrophil-recruiting and activating IL-8, which are transcriptionally regulated by $N F-\kappa B$, was shown to be decreased by several probiotic strains, including VSL\#3, Lactobacillus salivarius UCC118, Bifidobacterium infantis 35624 and Lactobacillus johnsonii $\mathrm{La}^{(423)}$. On the other hand, in a recent study, B. lactis Bb12 has been shown to target the toll-like receptor-2 signalling cascade, suggesting that probiotics themselves may trigger innate pro-inflammatory responses ${ }^{(424)}$. Similar pro-inflammatory effects by the activation of cytokines via toll-like receptor-2 are also reported for probiotic strains $L$. rhamnosus $\mathrm{GG}^{(425)}$ and L. casei Shirota ${ }^{(426)}$

The induction of protective responses by probiotics is highlighted by the capacity of some strains (or their components) to interact with gut-residing DC, e.g. via DC-SIGN, to induce their maturation and predominant secretion of IL-10, which is thought to favour the induction of regulatory T-cells ${ }^{(427)}$. In addition to regulatory T-cells, TGF- $\beta$ signalling in IECs plays a crucial role in maintaining mucosal immune homeostasis, as TGF- $\beta$ regulates expression of MHC class II molecules and activity of metalloproteinases ${ }^{(428)}$. Inhibition of TGF- $\beta$ signalling due to overexpression of the $\operatorname{Smad} 7$ protein, a negative regulator of TGF- $\beta$, in IECs was shown to perpetuate the production of pro-inflammatory cytokines ${ }^{(429)}$. VSL\#3 improved Th1-mediated experimental murine colitis by restoring the cytokine balance through induction of IL-10 and TGF- $\beta$ bearing regulatory T-cells ${ }^{(430)}$. Also, increased TGF- $\beta$ signalling induced by Bifidobacterium breve in preterm infants has been associated with reduced expression of Smad $7^{(431)}$.

\section{Effects in inflammatory conditions}

Inflammatory bowel diseases. A large body of evidence from both experimental animal work and clinical observations suggests that the enteric microflora may play an important role in driving the abnormal inflammatory response in the genetically susceptible host leading to chronic inflammation in IBD. This has led to the interest and increasing scientific evaluation of the use of probiotics as means to reconstitute microbial and immunological homeostasis. Although there are promising results for certain single probiotic strains in experimental models of colitis, the picture is more variable for the emerging human intervention studies using probiotic micro-organisms or combinations thereof as therapeutic interventions in IBD. Combinations of several probiotic strains have demonstrated efficacy in the control of pouchitis, an inflammation of the ileal-anal anastomosis, surgically produced after colorectomy for UC. In the present context, a randomised, placebo-controlled study with high dose of VSL\#3 was shown to be effective in the maintenance of antibioticinduced remission in chronic pouchitis ${ }^{(432)}$. By contrast, the use of single probiotic strains, such as L. rhamnosus GG or a combination of $L$. acidophilus and B. lactis did not show clinical improvement in acute active pouchitis ${ }^{(433)}$. In the treatment of acute $\mathrm{CD}$, in a small number of children with mild to moderate disease activity, L. rhamnosus GG in combination with prednisone and immunomodulatory drugs showed clinical improvement ${ }^{(434)}$. However, in adult patients with active CD L. rhamnosus GG was ineffective, although patients were on tapering steroid diet regimen and received antibiotics before starting the probiotic supplementation ${ }^{(435)}$. In the maintenance of surgically-induced remission, a few studies with divergent outcomes are reported. In a randomised placebo-controlled prospective study, oral administration of L. rhamnosus GG for 1 year could neither prevent Crohn's recurrent lesions after surgery nor reduce their severity ${ }^{(436)}$. By contrast, administration of VSL\#3 demonstrated efficacy in preventing post-operative recurrence when compared to mesalamine $^{(31)}$ up to 1 -year post-surgery. Two recent studies have shown no benefit from $L$. johnsonii La1 supplementation for prophylaxis of post-operative recurrence of inflammatory lesions in adult CD patients ${ }^{(437,438)}$. The therapeutic efficacy of E. coli Nissle 1917 in maintaining remission in UC patients has been demonstrated in human trials (equally efficacious as the standard mesalazine), while the same probiotic treatment lacked convincing data in active disease ${ }^{(439,440)}$. A randomised, open-label, multicentre trial showed that VSL\#3 in combination with low-dose balsalazide (a prodrug of mesalazine) was more effective than standard doses of basalazide or mesalazine monotherapy in the treatment of acute mild to moderate $\mathrm{UC}^{(441)}$. The efficacy of Lactobacillus GG in combination with mesazaline to sustain remission in patients with quiescent UC has been shown recently ${ }^{(42)}$. The role of 
bifidobacteria in medically induced remission of UC was evaluated in a small human trial showing less relapses in the bifidobacteria-supplemented patients during a 2-month follow-up. Inflammatory activity in the colon, assessed by NF-кB, IкB and pro-inflammatory cytokine analysis (IL-1 $\beta, \mathrm{TNF}-\alpha$ ), was significantly decreased in the probiotic treatment group ${ }^{(443)}$. Similarly, a fermented milk product with probiotic bifidobacteria had a positive effect on UC patients ${ }^{(444)}$. Finally, a randomised controlled pilot trial with B. longum in combination with a inulin-type prebiotics showed resolution of inflammation in UC patients and reduced inflammatory activity in the mucosal tissue (less TNF- $\alpha$, IL- $1 \alpha$, and defensins) ${ }^{(27)}$.

Taken together, the human study results demonstrate that the therapeutic intervention with probiotics in IBD is encouraging, but not as straightforward as expected from experiences with experimental animal models of colitis. Single probiotic strains have been demonstrated to successfully prevent or treat experimental colitis. However, the overall picture of probiotic therapy in human IBD is by far more complex. To date only a few randomised, double-blind placebo-controlled trials are published, which (so far) impose the conclusion that high doses of probiotics and most likely a combination of different lactobacilli and bifidobacteria (or prebiotics) are more effective in decreasing clinical inflammatory score and maintaining patients in medically induced remission than a single probiotic strain. Future research might identify clinical phenotypes of IBD patients (based on insights in genetic predisposition, microbiota asset and immune response) that are likely to respond to probiotic therapy. As a result of the emerging understanding of microbial activities in different parts of the intestine (in health and disease) and increasing scientific evidence of probiotic mechanisms, an educated and targeted selection of specific probiotic strains and combinations thereof will be possible in the future to apply probiotic therapy in conjunction with medical treatments to maintain clinical remission.

Rheumatoid arthritis. The treatment of experimental adjuvant arthritis with a combination of methotrexate and lyophilised Enterococcus faecium enriched with organic selenium was shown to be beneficial in reducing inflammation (serum albumin, serum nitrite/nitrate concentrations, changes in hind paw swelling, arthrogram score) and bone erosion in rats $^{(445)}$. However, the probiotic alone was not effective. One small-scale human trial on L. rhamnosus GG in human RA did not show a clinical benefit ${ }^{(446)}$.

Atopic eczema. Studies with animal models reveal that several lactobacilli strains including plantarum and casei have the potential to inhibit pro-allergic immune responses ${ }^{(447-450)}$. Infants with atopy show a different profile of gut microflora than non-atopic controls ${ }^{(64,451)}$ and a low number of bifidobacteria and lactobacilli predicted atopy development in Finnish infants ${ }^{(396,452)}$. Studies in pregnant women and infants have demonstrated that probiotics (L. casei GG) lower the risk of development of atopic eczema in infants ${ }^{(452-455)}$, this effect being associated with lowered levels of inflammatory markers in faeces. Rosenfeldt et al. ${ }^{(457)}$ showed that the combination of L. rhamnosus and L. reuteri reduced atopy severity and serum eosinophil cationic protein in 13-year-old atopic children.

$C V D$. Probiotic interventions in CVD are restricted to a few reports on modulation of risk factors associated with atherosclerosis, such as antioxidant defences or atherosclerotic lipid profiles. Hlivak et al. ${ }^{(458)}$ showed a reduction in LDL cholesterol after administration of Enterococcus faecium M-74 enriched with selenium. No significant alterations in HDL and TAG were observed. A second study reported a reduction in CVD risk factors (systolic blood pressure; leptin, fibrinogen, F(2)-isoprostanes, IL-6) in hyper-cholesterolaemic patients (heavy smokers) after consumption of L. plantarum 299v. Monocytes from the probiotic-treated group showed significantly reduced adhesion to native and stimulated human umbilical vein endothelial cells ${ }^{(459)}$.

\section{Conclusions}

Inflammation is a stereotypical physiological response to infections and tissue injury; it initiates pathogen killing as well as tissue repair processes and helps to restore homeostasis at infected or damaged sites. Acute inflammatory reactions are usually self-limiting and resolve rapidly. This involves the activation of negative feedback mechanisms such as the secretion of immunoregulatory cytokines (e.g. IL-10 and TGF- $\beta$ ), inhibition of pro-inflammatory signalling cascades, receptor shedding (e.g. TNF-R) and activation of regulatory cells. Thus, regulated inflammatory responses are essential to remain healthy and maintain homeostasis. However, inflammatory responses that fail to regulate themselves can become chronic and contribute to the perpetuation and progression of disease.

Characteristics typical of chronic inflammatory responses underlying the pathophysiology of several disorders include loss of barrier function, responsiveness to a normally benign stimulus, infiltration of inflammatory cells into compartments where they are not normally found in such high numbers, and overproduction of oxidants, cytokines, chemokines, eicosanoids and matrix metalloproteinases (Table 1). The levels of these mediators amplify the inflammatory response, are destructive and contribute to the clinical symptoms. Why resolution of inflammation is absent or abnormal in so many pathophysiological processes remains largely unknown.

Different factors including Western lifestyle (pollution, stress) and diet (low intake of fruits and vegetables, polyphenols and other antioxidants) have been postulated to reduce the efficiency of antioxidant defences, shifting the redox balance and thus increase the risk of inflammatory responses becoming chronic (Fig. 6). This may partially explain why many inflammatory disorders are more prevalent in Western countries and that in many cases prevalence increases with the level of westernisation $^{(460)}$.

Various dietary components have been suggested to impact on inflammatory conditions, but the number of studies assessing therapeutic benefits of dietary interventions in established inflammatory disorders is still fairly limited. There is good evidence of efficacy of $n$-3 PUFA in RA with less strong evidence in $\mathrm{CD}$ and psoriasis and rather weak evidence in UC, asthma and eczema (Table 3). These fatty acids are also beneficial in established CVD, but the extent to which this is attributable to their anti-inflammatory effects is not clear. Dietary antioxidants represent a crucial line of defence against oxidative and inflammatory insult common to the development of many pathological disorders and a potentially protective role of dietary antioxidants in disease prevention is supported by much basic scientific evidence. The common 

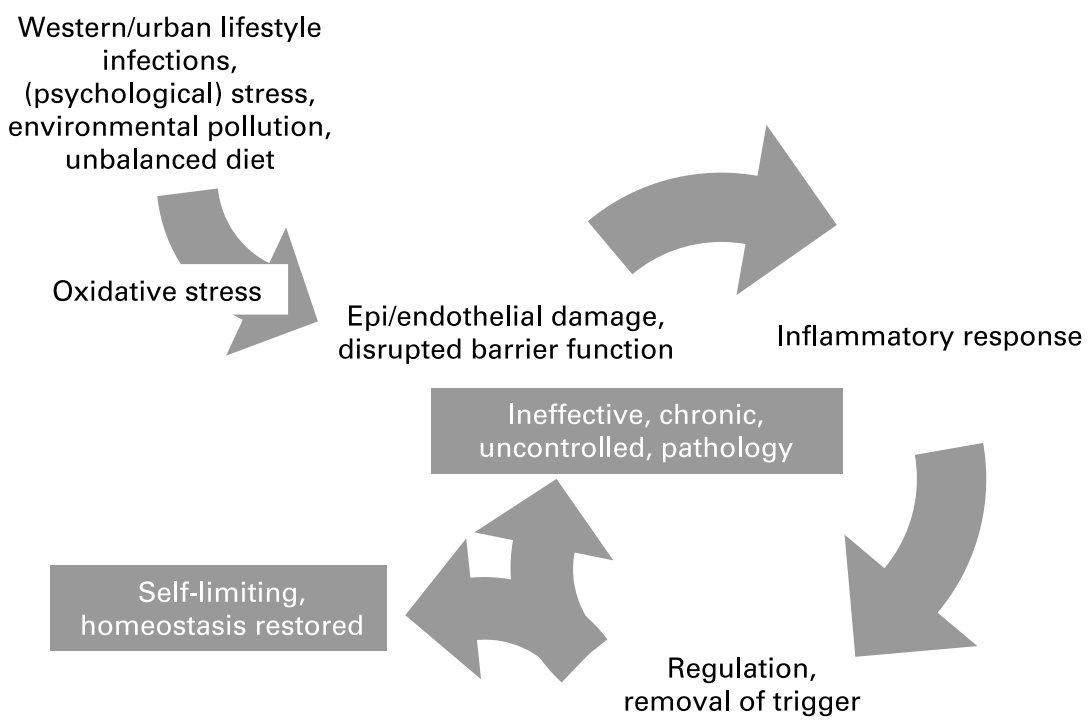

Fig. 6. Proposed link between Western lifestyle and chronic inflammation. Different factors associated with a Western lifestyle such as pollution, (psychological) stress and an unbalanced diet (low intake of fruits and vegetables, polyphenols and other antioxidants) reduce the efficiency of antioxidant defences, shifting the redox balance thus increasing the risk of inflammatory responses becoming chronic.

mechanism of oxidative stress development in most of the here-described pathologies make the role of dietary antioxidants crucial for an optimal preventive action. Despite these considerations, trials in patients suggest little clinical benefit from antioxidant vitamins and polyphenolics in the disorders considered here, although there is some evidence for benefit from vitamin $\mathrm{E}$ in RA (Table 3). In order to optimise the levels of protection against oxidative and inflammatory stress, the concept of antioxidant efficiency should be re-evaluated investigating the synergistic interactions among

Table 3. Summary of the effects of VLC $n-3$ PUFA, antioxidant vitamins, flavonoids, prebiotics and probiotics on immune/ inflammatory markers and conditions

\begin{tabular}{|c|c|c|c|c|c|}
\hline Effect on & VLC $n-3$ PUFA & Antioxidant vitamins & Flavonoids & Prebiotics & Probiotics \\
\hline Epithelial barrier & ? & ? & ? & Yes (gut) & Yes (gut) \\
\hline \multicolumn{6}{|l|}{ Cell function } \\
\hline Th1 $v$. Th2 balance & Yes & Yes & Yes & Yes & Yes \\
\hline $\mathrm{T}$ cell function & Yes & Yes & Yes & Yes & Yes \\
\hline Treg cell function & $?$ & $?$ & $?$ & $?$ & Yes \\
\hline NK cell function & ? & ? & Yes & Yes & Yes \\
\hline Dendritic cell function & Yes & Yes & ?Yes & Yes & Yes \\
\hline Macrophage function & Yes & Yes & Yes & Yes & Yes \\
\hline PMN function & Yes & Yes & Yes & Yes & Yes \\
\hline \multicolumn{6}{|l|}{ Cellular recruitment } \\
\hline Adhesion molecule expression & Yes & Yes & Yes & ? & ? \\
\hline Chemokine production & Yes & Yes & Yes & ? & ? \\
\hline \multicolumn{6}{|l|}{ Inflammatory mediator production } \\
\hline Inflammatory cytokines & Yes & Yes & Yes & Yes & Yes \\
\hline Arachidonic acid-derived eicosanoids & Yes & Yes & Yes & ?Yes & $?$ \\
\hline Matrix metalloproteinases & Yes & $?$ & Yes & ? & ? \\
\hline \multicolumn{6}{|l|}{ Transcription factors } \\
\hline $\mathrm{NF}-\kappa \mathrm{B}$ & Yes & Yes & Yes & Yes & Yes \\
\hline PPAR- $\gamma$ & Yes & $?$ & Yes & ? & ? \\
\hline Others & Yes & Yes & Yes & ? & ? \\
\hline \multicolumn{6}{|l|}{ Human disease outcome } \\
\hline Crohns Disease & ?Yes & ? & ? & Yes & Yes \\
\hline Ulcerative colitis & ?Yes & ? & ? & Yes & Yes \\
\hline COPD & ?Yes & Yes & ? & $?$ & ? \\
\hline Asthma & ?Yes & ?Yes & $?$ & $?$ & $?$ \\
\hline Eczema & ?Yes & $?$ & ? & Yes & Yes \\
\hline Psoriasis & Yes & ? & ? & $?$ & $?$ \\
\hline Rheumatoid arthritis & Yes & ? & ? & $?$ & ? \\
\hline Atherosclerosis & Yes & ?Yes & ?Yes & ? & ? \\
\hline
\end{tabular}

Yes indicates an effect has been demonstrated; ?Yes indicates effects on a clinical condition have been shown but that these are not conclusive ? indicates not known.

Th, helper T-cell; NK, natural killer; PMN, polymorphonuclear cell; COPD, chronic obstructive pulmonary disease. 


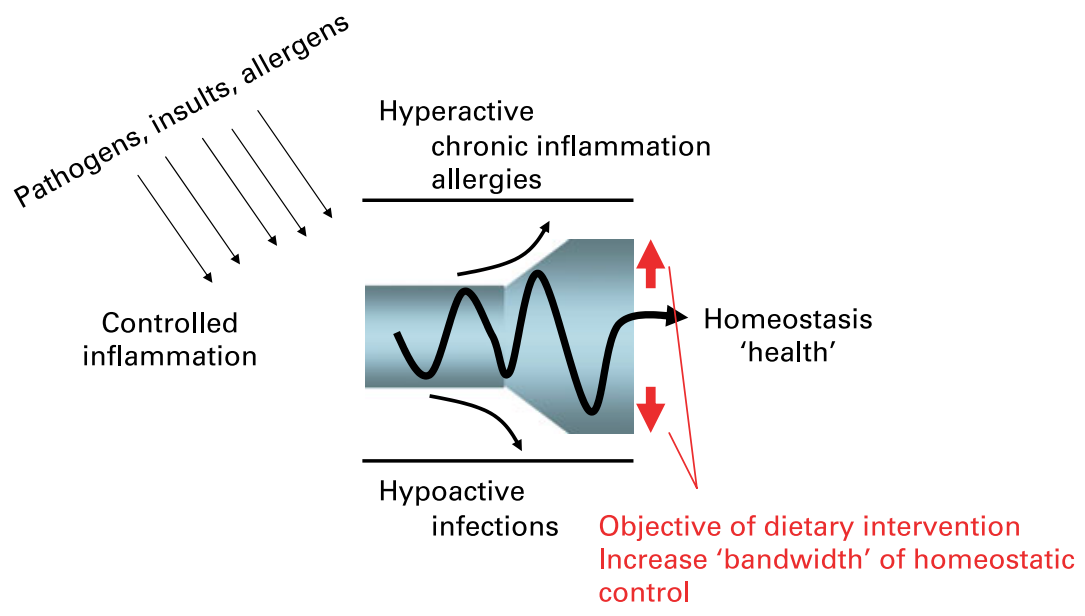

Fig. 7. Concept of how nutrients might act in an anti-inflammatory manner. By reinforcing the regulatory pathways controlling inflammatory responses, nutrition may contribute to the robustness of homeostatic control and may help to reduce the risk that acute inflammatory responses persist as uncontrolled chronic inflammatory responses. Nutrition may thus help to widen the boundaries within which the inflammatory responses can deal with the challenges posed by exposure to pathogens, allergens, toxins, tissue damage, etc., without spiralling into degenerative chronic inflammatory responses.

components of the redox network of the body. Efforts should be focused on the identification of the composition of redox network and of the mechanism(s) underlying its homeostatic modulation. The link between inflammatory and antioxidant status needs to be more deeply investigated with well-tailored in vivo studies. Particular attention needs to be paid towards the interactions between the two systems in healthy subjects and during the different phases of the inflammatory response in the development of pathologies.

The intestinal flora is in intimate contact with the most developed immunological organ of the human body imbedded in the intestinal membranes. There is a continuous intensive interaction established between the intestinal bacterial ecosystem and the host. The composition of this ecosystem can be improved either by selectively stimulating the resident bacterial populations that contribute to the protection against inflammatory processes (with prebiotics) or by adding external bacteria that are known to protect against inflammation (certain probiotics). Another important aspect is to locally stimulate the metabolic activity in a selective way (with prebiotics) so as to optimise the interaction with the immune system. There is evidence that consumption of prebiotics leads to clinical improvement in $\mathrm{UC}$ and $\mathrm{CD}$, with limited or no evidence in the other conditions described here (Table 3 ). There is good evidence that probiotics bring about clinically relevant improvements in UC and CD and protect against development of atopic dermatitis in infancy (Table 3 ). There is little or no evidence of efficacy in the other conditions described here. It is possible that nutrition may have a bigger impact on prevention rather than treatment of chronic inflammatory conditions. Studies with different dietary components in various models and clinical settings have demonstrated that dietary compounds modulate pathways involved in controlling inflammation including intracellular signalling pathways, transcription factor activity and generation of inflammatory mediators (Table 3). Bioavailability and tissue accumulation of antioxidant vitamins and $n-3$ PUFA are not a problem, but low bioavailability of flavanoids and the lack of studies showing a specific accumulation of flavonoids in blood and target tissues raise concerns about their role as anti-inflammatory agents in vivo. More studies and a larger body of evidence are needed for these phytochemicals.

By reinforcing the regulatory pathways controlling inflammatory responses, nutrition may contribute to the robustness of homeostatic control and may help to reduce the risk that acute inflammatory responses, which initiate pathogen killing as well as tissue repair processes, derail into uncontrolled chronic inflammatory responses that contribute to perpetuation and progression of disease (Fig. 7). Nutrition may thus help to widen the boundaries within which the inflammatory responses can deal with the challenges posed by exposure to pathogens, allergens, toxins, tissue damage, etc., without spiralling into degenerative chronic inflammatory responses (Fig. 7). Among the dietary components considered here, long-chain $n$-3 PUFA, antioxidant vitamins, plant flavonoids, prebiotics and probiotics all have the potential to modulate predisposition to chronic inflammatory conditions and may have a role in their therapy.

There is a clear need for more good-quality studies assessing the impact of nutrition and specific nutrients on chronic inflammatory responses. Ideally, such studies should assess relevant clinical symptoms of chronic inflammation as well as selected markers of inflammation. Not only will this help to understand the underlying mechanism, but it will also help to predict which other inflammatory conditions may benefit from particular (combinations of) anti-inflammatory dietary compounds. The putative effect of nutritional compounds in strengthening the regulatory networks controlling inflammatory responses should be studied in more detail using conditions and models in which a stress is applied to the homeostatic control of inflammation.

\section{Acknowledgements}

This work was commissioned by the Nutrition and Immunity Task Force of the European branch of the International Life Sciences Institute (ILSI Europe). Industry members of this task force are: FrieslandCampina, Groupe Danone, Nestlé, Seven Seas, Südzucker/BENEO Group, Royal Cosun, 
Unilever, Valio, Yakult. The opinions expressed herein are those of the authors and do not necessarily represent the views of ILSI Europe.

\section{Author contributions}

Authors drafted sections of the review as follows: F. G. (Gut: Celiac disease), S. M. (Gut: Inflammatory bowel diseases), M. L. (Lungs: Airways and allergic inflammation), W. v. E. (Joints: Rheumatoid arthritis), P. S. F. (Skin: Atopic eczema and psoriasis), G. A. F. (The vascular wall: CVD), G. S. F. (Adipose tissue: Obesity), P. C. C. (Fatty acids), B. M. W.- R. (Antioxidant vitamins), M. S. (Flavonoids), J. v. L. (Prebiotics), J. Z. and S. B. (Probiotics). All authors contributed to discussions at the Workshop and had input into the final version of the review. P. C. C. chaired the workshop and led the writing of the review.

\section{Conflict of interest statement}

P. C. C. has research funding from Beneo-Orafti and B. Braun Melsungen, at the time of drafting the review was a consultant to Equazen and Royal Dutch Numico and has received speaking fees from Solvay Healthcare, B. Braun Melsungen, Fresenius Kabi and Seven Seas Limited. R. A. is an employee of Unilever. J.- M. A. and R. B.- S. are employees of Danone Vitapole. S. B. is an employee of Nestle. G. A. F. has received speaking fees from Pfizer, Fournier, Astra-Zeneca and Roche. G. F. is an employee of Curax. F. G. is a member of the Beneo Scientific Committee sponsored by Orafti and of the Cimizia Advisory Board sponsored by UCB Pharma. P. D. M. is an employee of Sensus. At the time of the Workshop, L. M. was an employee of Royal Numico, now known as Danone Research Centre for Specialised Nutrition. M. S. has research funding from Coca-Cola and BPW. J. v. L. is an employee of Südzucker/BENEO Group. W. V. D. is an employee of Seven Seas Limited. S. V. is an employee of ILSI Europe. B. M. W.- R. has a research collaboration with DSM Nutritional Products. J. Z. is an employee of Yakult. P. S. F., G. S. F., S. M. and W. v. E. have no conflicts of interest to declare.

\section{References}

1. Aderem A \& Ulevitch RJ (2000) Toll-like receptors in the induction of the innate immune response. Nature 406, $782-787$.

2. Elewaut D, DiDonato JA, Kim JM, et al. (1999) NF-kappa B is a central regulator of the intestinal epithelial cell innate immune response induced by infection with enteroinvasive bacteria. J Immunol 163, 1457-1466.

3. Maaser C \& Kagnoff MF (2002) Role of the intestinal epithelium in orchestrating innate and adaptive mucosal immunity. Z Gastroenterol 40, 525-529.

4. Haller D, Bode C, Hammes WP, et al. (2000) Non-pathogenic bacteria elicit a differential cytokine response by intestinal epithelial cell/leucocyte co-cultures. Gut 47, 79-87.

5. Borruel N, Casellas F, Antolin M, et al. (2003) Effects of nonpathogenic bacteria on cytokine secretion by human intestinal mucosa. Am J Gastroenterol 98, 865-870.

6. Brandtzaeg P, Farstad IN, Johansen FE, et al. (1999) The B-cell system of human mucosae and exocrine glands. Immunol Rev 171, 45-87.
7. Shanahan F (1994) The intestinal immune system. In Physiology of the Gastrointestinal Tract, pp. 643-684 [LR Johnson, J Christensen, E Jacobsen and JH Walsh, editors]. New York: Raven Press.

8. Scott H, Rognum TO, Midvedt T, et al. (1985) Age related changes of human serum antibodies to dietary and colonic bacterial antigens measured by an enzyme linked immunosorbent assay. Acta Pathol Microbiol Immunol Scand 93, 65-70.

9. Brandtzaeg PE (2002) Current understanding of gastrointestinal immunoregulation and its relation to food allergy. Ann $N$ Y Acad Sci 964, 13-45.

10. Cummings JH, Antoine JM, Azpiroz F, et al. (2004) PASSCLAIM- Gut health and immunity. Eur J Nutr 43, II118-II173.

11. Mulder CJ \& Cellier C (2005) Coeliac disease: changing views. Best Pract Res Clin Gastroenterol 19, 313-321.

12. Marsh MN (1992) Gluten, major histocompatibility complex, and the small intestine. A molecular and immunobiologic approach to the spectrum of gluten sensitivity ('celiac sprue'). Gastroenterology 102, 330-354.

13. Rostom A, Dube C, Cranney A, et al. (2005) The diagnostic accuracy of serologic tests for celiac disease: a systematic review. Gastroenterology 128, S38-S46.

14. Rewers M (2005) Epidemiology of celiac disease: what are the prevalence, incidence, and progression of celiac disease? Gastroenterology 128, S47-S51.

15. Dube C, Rostom A, Sy R, et al. (2005) The prevalence of celiac disease in average-risk and at-risk Western European populations: a systematic review. Gastroenterology 128, S57-S67.

16. Liu E, Rewers M \& Eisenbarth GS (2005) Genetic testing: who should do the testing and what is the role of genetic testing in the setting of celiac disease? Gastroenterology 128, S33-S37.

17. Ivarsson A (2005) The Swedish epidemic of coeliac disease explored using an epidemiological approach - some lessons to be learnt. Best Pract Res Clin Gastroenterol 19, 426-440.

18. Kagnoff MF (2005) Overview and pathogenesis of celiac disease. Gastroenterology 128, S10-S18.

19. Sollid LM \& Khosla C (2005) Future therapeutic options for celiac disease. Nat Clin Pract Gastroenterol Hepatol 2, $140-147$

20. Duchmann R, Kaiser I, Hermann E, et al. (1995) Tolerance exists towards resident intestinal flora but is broken in active inflammatory bowel disease (IBD). Clin Exp Immunol 102, 448-455.

21. Farrell RJ \& Peppercorn MA (2002) Ulcerative colitis. Lancet 359, 331-340.

22. Shanahan F (2002) Crohn's disease. Lancet 359, 62-69.

23. Carter MJ, Lobo AJ \& Travis SP (2004) Guidelines for the management of inflammatory bowel disease in adults. Gut 53, Suppl. 5, V1-V16.

24. Ogura Y, Bonen DK, Inohara N, et al. (2001) A frameshift mutation in NOD2 associated with susceptibility to Crohn's disease. Nature 411, 603-606.

25. Taurog JD, Richardson JA, Croft JT, et al. (1994) The germfree state prevents development of gut and joint inflammatory disease in HLA-B27 transgenic rats. J Exp Med 180, 2359-2364.

26. Macfarlane S, Furrie E, Kennedy A, et al. (2005) Mucosal bacteria in ulcerative colitis. Br J Nutr 93, S67-S72.

27. Furrie E, Macfarlane S, Kennedy A, et al. (2005) Synbiotic therapy (Bifidobacterium longum/Synergy 1) initiates resolution of inflammation in patients with active ulcerative colitis: a randomised controlled pilot trial. Gut 54, 242-249.

28. Seksik P, Rigottier-Gois L, Gramet G, et al. (2003) Alterations of the dominant faecal bacterial groups in patients with Crohn's disease of the colon. Gut 52, 237-242. 
29. Macfarlane S, Furrie E, Cummings JH, et al. (2004) Chemotaxonomic analysis of bacterial populations colonizing the rectal mucosa in patients with ulcerative colitis. Clin Infect Dis 38, 1690-1699.

30. Famularo G, Moretti S, Marcellini S, et al. (1997) Stimulation of immunity by probiotics. In Probiotics: therapeutic and Other Beneficial Effects, pp. 133-161 [R Fuller, editor]. London: Chapman \& Hall.

31. Gionchetti P, Rizzello F, Venturi A, et al. (2000) Oral bacteriotherapy as maintenance treatment in patients with chronic pouchitis: a double-blind, placebo-controlled trial. Gastroenterology 119, 305-309.

32. Musso A, Dentelli P, Carlino A, et al. (2005) Signal transducers and activators of transcription 3 signaling pathway: an essential mediator of inflammatory bowel disease and other forms of intestinal inflammation. Inflamm Bowel Dis 11, 91-98.

33. von Lampe B, Barthel B, Coupland SE, et al. (2000) Differential expression of matrix metalloproteinases and their tissue inhibitors in colon mucosa of patients with inflammatory bowel disease. Gut 47, 63-73.

34. Rahman I, Biswas SK \& Kode A (2006) Oxidant and antioxidant balance in the airways and airway diseases. Eur J Pharmacol 533, 222-239.

35. Hargreave FE \& Parameswaran K (2006) Asthma, COPD and bronchitis are just components of airway disease. Eur Respir $J$ 28, 264-267.

36. Elias J (2004) The relationship between asthma and COPD. Lessons from transgenic mice. Chest 126, 111S-116S.

37. Agusti AG, Noguera A, Sauleda J, et al. (2003) Systemic effects of chronic obstructive pulmonary disease. Eur Respir $J$ 21, 347-360.

38. Agusti AG (2005) Systemic effects of chronic obstructive pulmonary disease. Proc Am Thorac Soc 2, 367-370.

39. Gan WQ, Man SF, Senthilselvan A, et al. (2004) Association between chronic obstructive pulmonary disease and systemic inflammation: a systematic review and a meta-analysis. Thorax 59, 574-580.

40. Phipatanakul W (2005) Allergic rhinoconjunctivitis: epidemiology. Immunol Allergy Clin North Am 25, 263-281.

41. Scadding G \& Mitchell D (2004) Rhinitis and sinusitis. In Clinical Respiratory Medicine, pp. 339-350 [RK Albert, SG Spiro and JR Jett, editors]. Philadelphia, PA: Mosby.

42. Strachan D, Sibbald B, Weiland S, et al. (1997) Worldwide variations in prevalence of symptoms of allergic rhinoconjunctivitis in children: the International Study of Asthma and Allergies in Childhood (ISAAC). Pediatr Allergy Immunol 8, $161-176$.

43. Maziak W, Behrens T, Brasky TM, et al. (2003) Are asthma and allergies in children and adolescents increasing? Results from ISAAC phase I and phase III surveys in Munster, Germany. Allergy 58, 572-579.

44. Postma DS, Kerstjens HAM \& Ten Hacken NHT (2004) Asthma: Epidemiology and risk factors. In Clinical Respiratory Medicine, pp. 457-465 [RK Albert, SG Spiro and JR Jett, editors]. Philadelphia, PA: Mosby.

45. The International Study of Asthma and Allergies in Childhood (ISAAC) Steering Committee (1998) Worldwide variations in the prevalence of asthma symptoms: the International Study of Asthma and Allergies in Childhood (ISAAC). Eur Respir J 12, $315-335$.

46. The International Study of Asthma and Allergies in Childhood (ISAAC) Steering Committee (1998) Worldwide variation in prevalence of symptoms of asthma, allergic rhinoconjunctivitis, and atopic eczema: ISAAC. Lancet 351, 1225-1232.

47. Russel G (2006) The childhood asthma epidemic. Thorax 61, 276-278.
48. Zollner IK, Weiland SK, Piechotowski I, et al. (2005) No increase in the prevalence of asthma, allergies, and atopic sensitisation among children in Germany: 1992-2001. Thorax 60, $545-548$.

49. Lodrup Carlsen KC, Haland G, Devulapalli CS, et al. (2006) Asthma in every fifth child in Oslo, Norway: a 10-year follow up of a birth cohort study. Allergy 61, 454-460.

50. Murray CJ \& Lopez AD (1997) Mortality by cause for eight regions of the world: Global Burden of Disease Study. Lancet 349, 1269-1276.

51. MacNee W (2004) Chronic obstructive pulmonary disease: Epidemiology, pathophysiology, and clinical evaluation. In Clinical Respiratory Medicine, pp. 415-435 [RK Albert, SG Spiro and JR Jett, editors]. Philadelphia, PA: Mosby.

52. Leung R \& Ho P (1994) Asthma, allergy, and atopy in three South-East Asian populations. Thorax 49, 1205-1210.

53. von Mutius E, Martinez FD, Fritzsch C, et al. (1994) Prevalence of asthma and atopy in two areas of West and East Germany. Am J Respir Crit Care Med 149, 358-364.

54. Strachan DP (1989) Hay fever, hygiene, and household size. BMJ 299, 1259-1260.

55. Umetsu DT (2004) Flu strikes the hygiene hypothesis. Nat Med 10, 232-234.

56. Umetsu DT (2005) Revising the immunological theories of asthma and allergy. Lancet 365, 98-100.

57. Cullinan P (2006) Childhood allergies, birth order and family size. Thorax 61, 3-5.

58. von Mutius E (2000) The environmental predictors of allergic disease. J Allergy Clin Immunol 105, 9-19.

59. von Mutius E, Martinez FD, Fritzsch C, et al. (1994) Skin test reactivity and number of siblings. BMJ 308, 692-695.

60. Ball TM, Castro-Rodriguez JA, Griffith KA, et al. (2000) Siblings, day-care attendance, and the risk of asthma and wheezing during childhood. $N$ Engl J Med 343, 538-543.

61. Riedler J, Braun-Fahrlander C, Eder W, et al. (2001) Exposure to farming in early life and development of asthma and allergy: a cross-sectional survey. Lancet 358, 1129-1133.

62. Floistrup H, Swartz J, Bergstrom A, et al. (2006) Allergic disease and sensitization in Steiner school children. J Allergy Clin Immunol 117, 59-66.

63. Platts-Mills T, Vaughan J, Squillace S, et al. (2001) Sensitisation, asthma, and a modified $\mathrm{Th} 2$ response in children exposed to cat allergen: a population-based cross-sectional study. Lancet 357, 752-756.

64. Bjorksten B, Naaber P, Sepp E, et al. (1999) The intestinal microflora in allergic Estonian and Swedish 2-year-old children. Clin Exp Allergy 29, 342-346.

65. McKeever TM, Lewis SA, Smith C, et al. (2002) The importance of prenatal exposures on the development of allergic disease: a birth cohort study using the West Midlands General Practice Database. Am J Respir Crit Care Med 166, 827-832.

66. Wong GW, Ko FW, Hui DS, et al. (2004) Factors associated with difference in prevalence of asthma in children from three cities in China: multicentre epidemiological survey. BMJ 329, 486.

67. Chen R, Hu Z \& Seaton A (2004) Eating more vegetables might explain reduced asthma symptoms. BMJ 328, 1380.

68. Mickleborough TD \& Rundell KW (2005) Dietary polyunsaturated fatty acids in asthma- and exercise-induced bronchoconstriction. Eur J Clin Nutr 59, 1335-1346.

69. Beuther DA \& Sutherland ER (2007) Overweight, obesity, and incident asthma: a meta-analysis of prospective epidemiologic studies. Am J Respir Crit Care Med 175, 661-666.

70. Shaaban R, Leynaert B, Soussan D, et al. (2007) Physical activity and bronchial hyperresponsiveness: European Community Respiratory Health Survey II. Thorax 62, 403-410.

71. Umetsu DT, McIntire JJ, Akbari O, et al. (2002) Asthma: an epidemic of dysregulated immunity. Nat Immunol 3, 715-720. 
72. Watson L, Margetts B, Howarth P, et al. (2002) The association between diet and chronic obstructive pulmonary disease in subjects selected from general practice. Eur Respir J 20, 313-318.

73. Kingma PS \& Whitsett JA (2006) In defense of the lung: surfactant protein A and surfactant protein D. Curr Opin Pharmacol 6, 277-283.

74. Van Wetering S, Hiemstra PS \& Rabe KF (2004) Asthma: cell biology. In Clinical Respiratory Medicine, pp. 467-473 [RK Albert, SG Spiro and JR Jett, editors]. Philadelphia, PA: Mosby.

75. Akbari O, Stock P, Meyer E, et al. (2003) Essential role of NKT cells producing IL-4 and IL-13 in the development of allergen-induced airway hyperreactivity. Nat $\operatorname{Med} \mathbf{9}, 582-588$.

76. Erin EM, Leaker BR, Nicholson GC, et al. (2006) The effects of a monoclonal antibody directed against tumour necrosis factor- $\alpha$ (TNF- $\alpha)$ in asthma. Am J Respir Crit Care Med 174, 763-771.

77. Ray A \& Cohn L (1999) Th2 cells and GATA-3 in asthma: new insights into the regulation of airway inflammation. J Clin Invest 104, 985-993.

78. Barrios RJ, Kheradmand F, Batts L, et al. (2006) Asthma: pathology and pathophysiology. Arch Pathol Lab Med 130, 447-451.

79. Steinman L (2007) A brief history of $\mathrm{T}(\mathrm{H}) 17$, the first major revision in the $\mathrm{T}(\mathrm{H}) 1 / \mathrm{T}(\mathrm{H}) 2$ hypothesis of $\mathrm{T}$ cell-mediated tissue damage. Nat Med 13, 139-145.

80. Hansen G, Berry G, DeKruyff RH, et al. (1999) Allergenspecific Th1 cells fail to counterbalance Th2 cell-induced airway hyperreactivity but cause severe airway inflammation. $J$ Clin Invest 103, 175-183.

81. Randolph DA, Carruthers CJ, Szabo SJ, et al. (1999) Modulation of airway inflammation by passive transfer of allergenspecific Th1 and Th2 cells in a mouse model of asthma. $J$ Immunol 162, 2375-2383.

82. Heaton T, Rowe J, Turner S, et al. (2005) An immunoepidemiological approach to asthma: identification of in vitro T-cell response patterns associated with different wheezing phenotypes in children. Lancet 365, 142-149.

83. Saetta M, Di SA, Turato G, et al. (2005) CD8 + T-lymphocytes in peripheral airways of smokers with chronic obstructive pulmonary disease. Am J Respir Crit Care Med 157, $822-826$.

84. van Oosterhout AJ \& Bloksma N (2005) Regulatory T-lymphocytes in asthma. Eur Respir J 26, 918-932.

85. Finotto S, Neurath MF, Glickman JN, et al. (2002) Development of spontaneous airway changes consistent with human asthma in mice lacking T-bet. Science 295, 336-338.

86. Nassenstein C, Schulte-Herbruggen O, Renz H, et al. (2006) Nerve growth factor: the central hub in the development of allergic asthma? Eur J Pharmacol 533, 195-206.

87. De Swert KO \& Joos GF (2006) Extending the understanding of sensory neuropeptides. Eur J Pharmacol 533, 171-181.

88. Tracey KJ (2005) Fat meets the cholinergic antiinflammatory pathway. J Exp Med 202, 1017-1021.

89. Van Eerdewegh P, Little RD, Dupuis J, et al. (2002) Association of the ADAM33 gene with asthma and bronchial hyperresponsiveness. Nature 418, 426-430.

90. Fahy JV, Corry DB \& Boushey HA (2000) Airway inflammation and remodeling in asthma. Curr Opin Pulmonary Med 6, 15-20.

91. Lee KS, Park HS, Park SJ, et al. (2006) An antioxidant modulates expression of receptor activator of NF-kappaB in asthma. Exp Mol Med 38, 217-229.

92. Fujisawa $\mathrm{T}$ (2005) Role of oxygen radicals on bronchial asthma. Curr Drug Targets Inflamm Allergy 4, 505-509.

93. Dworski R (2000) Oxidant stress in asthma. Thorax 55, Suppl. 2, S51-S53.
94. Chan RC, Wang M, Li N, et al. (2006) Pro-oxidative diesel exhaust particle chemicals inhibit LPS-induced dendritic cell responses involved in T-helper differentiation. J Allergy Clin Immunol 118, 455-465.

95. Vinzents PS, Moller P, Sorensen M, et al. (2005) Personal exposure to ultrafine particles and oxidative DNA damage. Environ Health Perspect 113, 1485-1490.

96. Lee CG, Link H, Baluk P, et al. (2004) Vascular endothelial growth factor (VEGF) induces remodeling and enhances TH2-mediated sensitization and inflammation in the lung. Nat Med 10, 1095-1103.

97. Kharitonov SA \& Barnes PJ (2002) Biomarkers of some pulmonary diseases in exhaled breath. Biomarkers 7, 1-32.

98. Kostikas K, Papatheodorou G, Psathakis K, et al. (2003) Oxidative stress in expired breath condensate of patients with COPD. Chest 124, 1373-1380.

99. Paredi P, Kharitonov SA \& Barnes PJ (2002) Analysis of expired air for oxidation products. Am J Respir Crit Care Med 166, S31-S37.

100. Shapiro SD (2003) Proteolysis in the lung. Eur Respir J Suppl 44, 30s-32s.

101. D'Armiento J, Dalal SS, Okada Y, et al. (1992) Collagenase expression in the lungs of transgenic mice causes pulmonary emphysema. Cell 71, 955-961.

102. van Eden W, van der Zee R \& Prakken B (2005) Heat-shock proteins induce $\mathrm{T}$-cell regulation of chronic inflammation. Nat Rev Immunol 5, 318-330.

103. van Amelsfort JM, Jacobs KM, Bijlsma JW, et al. (2004) $\mathrm{CD} 4(+) \mathrm{CD} 25(+)$ regulatory $\mathrm{T}$ cells in rheumatoid arthritis: differences in the presence, phenotype, and function between peripheral blood and synovial fluid. Arthritis Rheum 50, 2775-2785.

104. Lee DM \& Weinblatt ME (2001) Rheumatoid arthritis. Lancet 358, 903-911.

105. Pincus T \& Callahan LF (1989) Reassessment of twelve traditional paradigms concerning the diagnosis, prevalence, morbidity and mortality of rheumatoid arthritis. Scand $J$ Rheumatol Suppl 79, 67-96.

106. Silman AJ \& Pearson JE (2002) Epidemiology and genetics of rheumatoid arthritis. Arthritis Res 4, Suppl. 3, S265-S272.

107. Uhlig T \& Kvien TK (2005) Is rheumatoid arthritis disappearing? Ann Rheum Dis 64, 7-10.

108. Woo P \& Wedderburn LR (1998) Juvenile chronic arthritis. Lancet 351, 969-973.

109. de Kleer I, Wedderburn LR, Taams LS, et al. (2004) CD4 + $\mathrm{CD} 25$ bright regulatory $\mathrm{T}$ cells actively regulate inflammation in the joints of patients with the remitting form of juvenile idiopathic arthritis. J Immunol 172, 6435-6443.

110. de Kleer I, Kamphuis SM, Rijkers GT, et al. (2003) The spontaneous remission of juvenile idiopathic arthritis is characterized by $\mathrm{CD} 30+\mathrm{T}$ cells directed to human heat-shock protein 60 capable of producing the regulatory cytokine interleukin-10. Arthritis Rheum 48, 2001-2010.

111. Nielsen HE, Dorup J, Herlin T, et al. (1999) Epidemiology of juvenile chronic arthritis: risk dependent on sibship, parental income, and housing. J Rheumatol 26, 1600-1605.

112. van der Helm-van Mil AH, Wesoly JZ \& Huizinga TW (2005) Understanding the genetic contribution to rheumatoid arthritis. Curr Opin Rheumatol 17, 299-304.

113. Begovich AB, Carlton VE, Honigberg LA, et al. (2004) A missense single-nucleotide polymorphism in a gene encoding a protein tyrosine phosphatase (PTPN22) is associated with rheumatoid arthritis. Am J Hum Genet 75, 330-337.

114. van der Helm-van Mil AH, Verpoort KN, Breedveld FC, et al. (2005) Antibodies to citrullinated proteins and differences in clinical progression of rheumatoid arthritis. Arthritis Res Ther 7, R949-R958. 
115. Firestein GS (2003) Evolving concepts of rheumatoid arthritis. Nature 423, 356-361.

116. Panayi GS, Lanchbury JS \& Kingsley GH (1992) The importance of the $\mathrm{T}$ cell in initiating and maintaining the chronic synovitis of rheumatoid arthritis. Arthritis Rheum 35, 729-735.

117. Weyand CM, Seyler TM \& Goronzy JJ (2005) B cells in rheumatoid synovitis. Arthritis Res Ther 7, Suppl. 3, S9-S12.

118. Bridges SL (2004) Update on autoantibodies in rheumatoid arthritis. Curr Rheumatol Rep 6, 343-350.

119. Vossenaar ER \& van Venrooij WJ (2004) Citrullinated proteins: sparks that may ignite the fire in rheumatoid arthritis. Arthritis Res Ther 6, 107-111.

120. Edwards JC, Leandro MJ \& Cambridge G (2004) B lymphocyte depletion therapy with rituximab in rheumatoid arthritis. Rheum Dis Clin North Am 30, 393-403.

121. Firestein GS, varo-Gracia JM \& Maki R (1990) Quantitative analysis of cytokine gene expression in rheumatoid arthritis. J Immunol 144, 3347-3353.

122. Kinne RW, Brauer R, Stuhlmuller B, et al. (2000) Macrophages in rheumatoid arthritis. Arthritis Res 2, 189-202.

123. Kontoyiannis D \& Kollias G (2000) Fibroblast biology. Synovial fibroblasts in rheumatoid arthritis: leading role or chorus line? Arthritis Res 2, 342-343.

124. Ehrenstein MR, Evans JG, Singh A, et al. (2004) Compromised function of regulatory $\mathrm{T}$ cells in rheumatoid arthritis and reversal by anti-TNFalpha therapy. J Exp Med 200, 277-285.

125. Williams HC (2000) Epidemiology of atopic dermatitis. Clin Exp Dermatol 25, 522-529.

126. Krutmann J \& Grewe M (1996) Sequential activation of Th1 and Th2 cells in the immunopathogenesis of atopic eczema - the 2-phase model. Allergologie 19, 449-451.

127. Thepen T, Langeveld-Wildschut EG, Bihari IC, et al. (1996) Biphasic response against aeroallergen in atopic dermatitis showing a switch from an initial $\mathrm{TH} 2$ response to a TH1 response in situ: an immunocytochemical study. J Allergy Clin Immunol 97, 828-837.

128. Saito $\mathrm{S}$ (2000) Cytokine network at the feto-maternal interface. J Reprod Immunol 47, 87-103.

129. von Mutius E, Braun-Fahrlander C, Schierl R, et al. (2000) Exposure to endotoxin or other bacterial components might protect against the development of atopy. Clin Exp Allergy 30, $1230-1234$.

130. Gehring U, Bolte G, Borte M, et al. (2001) Exposure to endotoxin decreases the risk of atopic eczema in infancy: a cohort study. J Allergy Clin Immunol 108, 847-854.

131. Romagnani S (2004) The increased prevalence of allergy and the hygiene hypothesis: missing immune deviation, reduced immune suppression, or both? Immunology 112, 352-363.

132. Chan SC, Kim JW, Henderson WR Jr, et al. (1993) Altered prostaglandin E2 regulation of cytokine production in atopic dermatitis. J Immunol 151, 3345-3352.

133. Chan S, Henderson WR Jr, Li SH, et al. (1996) Prostaglandin $\mathrm{E} 2$ control of $\mathrm{T}$ cell cytokine production is functionally related to the reduced lymphocyte proliferation in atopic dermatitis. J Allergy Clin Immunol 97, 85-94.

134. Ena P, Madeddu P, Glorioso N, et al. (1985) High prevalence of cardiovascular diseases and enhanced activity of the reninangiotensin system in psoriatic patients. Acta Cardiol 40, 199-205.

135. Rocha-Pereira P, Santos-Silva A, Rebelo I, et al. (2001) Dislipidemia and oxidative stress in mild and in severe psoriasis as a risk for cardiovascular disease. Clin Chim Acta 303, 33-39.

136. Bernstein CN, Wajda A \& Blanchard JF (2005) The clustering of other chronic inflammatory diseases in inflammatory bowel disease: a population-based study. Gastroenterology 129, $827-836$.
137. Allen BR \& Littlewood SM (1983) The aetiology of psoriasis: clues provided by benoxaprofen. Br J Dermatol 109, Suppl. 25, 126-129.

138. Kragballe K \& Herlin T (1983) Benoxaprofen improves psoriasis. A double-blind study. Arch Dermatol 119, 548-552.

139. Anggard E (1994) Nitric oxide: mediator, murderer, and medicine. Lancet 343, 1199-1206.

140. Penn MS, Rangaswamy S, Saidel GM, et al. (1997) Macromolecular transport in the arterial intima: comparison of chronic and acute injuries. Am J Physiol 272, H1560-H1570.

141. Rangaswamy S, Penn MS, Saidel GM, et al. (1997) Exogenous oxidized low-density lipoprotein injures and alters the barrier function of endothelium in rats in vivo. Circ Res 80, 37-44.

142. Scalia R, Pearlman S, Campbell B, et al. (1996) Time course of endothelial dysfunction and neutrophil adherence and infiltration during murine traumatic shock. Shock 6, 177-182.

143. Kupatt C, Zahler S, Seligmann C, et al. (1996) Nitric oxide mitigates leukocyte adhesion and vascular leak after myocardial ischemia. J Mol Cell Cardiol 28, 643-654.

144. Ohara Y, Peterson TE \& Harrison DG (1993) Hypercholesterolemia increases endothelial superoxide anion production. $J$ Clin Invest 91, 2546-2551.

145. Steinberg D, Parthasarathy S, Carew TE, et al. (1989) Beyond cholesterol. Modifications of low-density lipoprotein that increase its atherogenicity. $N$ Engl J Med 320, 915-924.

146. Miller ER 3rd, Pastor-Barriuso R, Dalal D, et al. (2005) Metaanalysis: high-dosage vitamin $\mathrm{E}$ supplementation may increase all-cause mortality. Ann Intern Med 142, 37-46.

147. Bjelakovic G, Nikolova D, Gluud LL, et al. (2007) Mortality in randomized trials of antioxidant supplements for primary and secondary prevention: systematic review and meta-analysis. JAMA 297, 842-857.

148. Masuda J \& Ross R (1990) Atherogenesis during low level hypercholesterolemia in the nonhuman primate. I. Fatty streak formation. Arteriosclerosis 10, 164-177.

149. Masuda J \& Ross R (1990) Atherogenesis during low level hypercholesterolemia in the nonhuman primate. II. Fatty streak conversion to fibrous plaque. Arteriosclerosis 10, 178-187.

150. Stary HC (2000) Lipid and macrophage accumulations in arteries of children and the development of atherosclerosis. Am J Clin Nutr 72, 1297S-1306S.

151. Stary HC (2000) Natural history and histological classification of atherosclerotic lesions: an update. Arterioscler Thromb Vasc Biol 20, 1177-1178.

152. Ross R (1993) The pathogenesis of atherosclerosis: a perspective for the 1990s. Nature 362, 801-809.

153. Lendon CL, Davies MJ, Born GV, et al. (1991) Atherosclerotic plaque caps are locally weakened when macrophages density is increased. Atherosclerosis 87, 87-90.

154. George SJ (1998) Tissue inhibitors of metalloproteinases and metalloproteinases in atherosclerosis. Curr Opin Lipidol 9, 413-423.

155. Katsuda S, Boyd HC, Fligner C, et al. (1992) Human atherosclerosis. III. Immunocytochemical analysis of the cell composition of lesions of young adults. Am J Pathol 140, 907-914.

156. Hansson GK (2005) Inflammation, atherosclerosis, and coronary artery disease. $N$ Engl J Med 352, 1685-1695.

157. Rifai N \& Ridker PM (2002) Inflammatory markers and coronary heart disease. Curr Opin Lipidol 13, 383-389.

158. Hulthe J, Wikstrand J \& Fagerberg B (2001) Relationship between C-reactive protein and intima-media thickness in the carotid and femoral arteries and to antibodies against oxidized low-density lipoprotein in healthy men: The Atherosclerosis and Insulin Resistance (AIR) Study. Clin Sci 100, 371-378. 
159. Mohamed-Ali V \& Coppack S (2005) Adipose tissue derived factors. In Cardiovascular Disease: Diet, Nutrition and Emerging Risk Factors, pp. 160-176 [S Stanner, editor]. Blackwell Publishers: Oxford.

160. Vaudo G, Marchesi S, Gerli R, et al. (2004) Endothelial dysfunction in young patients with rheumatoid arthritis and low disease activity. Ann Rheum Dis 63, 31-35.

161. Bruce IN, Urowitz MB, Gladman DD, et al. (2003) Risk factors for coronary heart disease in women with systemic lupus erythematosus: The Toronto Risk Factor Study. Arthritis Rheum 48, 3159-3167.

162. Kannel WB, D'Agostino RB, Sullivan L, et al. (2004) Concept and usefulness of cardiovascular risk profiles. Am Heart $J$ 148, $16-26$.

163. Unal B, Critchley JA \& Capewell S (2005) Modelling the decline in coronary heart disease deaths in England and Wales, 1981-2000: comparing contributions from primary prevention and secondary prevention. BMJ 331, 614 .

164. Unal B, Critchley JA \& Capewell S (2005) Small changes in United Kingdom cardiovascular risk factors could halve coronary heart disease mortality. J Clin Epidemiol 58, 733-740.

165. Lampe FC, Morris RW, Walker M, et al. (2005) Trends in rates of different forms of diagnosed coronary heart disease, 1978 to 2000: prospective, population based study of British men. BMJ 330, 1046.

166. Report of the Cardiovascular Review Group, Committee on Medical Aspects of Food Policy (2004) Nutritional Aspects of Cardiovascular Disease. London: HMSO.

167. Zatonski WA \& Willett W (2005) Changes in dietary fat and declining coronary heart disease in Poland: population based study. BMJ 331, 187-188.

168. British Heart Foundation Health Promotion Research Group (2003) Coronary Heart Disease Statistics. London: British Heart Foundation.

169. Frayn K \& Stanner S (2007) The aetiology and epidemiology of cardiovascular disease. In Cardiovascular Disease: Diet, Nutrition and Emerging Factors, pp. 1-21 [S Stanner, editor]. Oxford: Blackwell Publishing.

170. Baigent C, Keech A, Kearney PM, et al. (2005) Efficacy and safety of cholesterol-lowering treatment: prospective metaanalysis of data from 90,056 participants in 14 randomised trials of statins. Lancet 366, 1267-1278.

171. Baigent C, Sudlow C, Collins R, et al. (2002) Collaborative meta-analysis of randomised trials of antiplatelet therapy for prevention of death, myocardial infarction, and stroke in high risk patients. $B M J 324,71-86$.

172. Taylor-Robinson D \& Boman J (2005) The failure of antibiotics to prevent heart attacks. BMJ 331, 361-362.

173. Calder PC (2006) $n$-3 Polyunsaturated fatty acids, inflammation, and inflammatory diseases. Am J Clin Nutr 83, 1505S-1519S.

174. Thies F, Garry JM, Yaqoob P, et al. (2003) Association of $n-3$ polyunsaturated fatty acids with stability of atherosclerotic plaques: a randomised controlled trial. Lancet 361, 477-485.

175. GISSI Prevenzione (1999) Dietary supplementation with $n$-3 polyunsaturated fatty acids and vitamin $\mathrm{E}$ after myocardial infarction: results of the GISSI-Prevenzione trial. Lancet 354, 447-455.

176. Marchioli R, Barzi F, Bomba E, et al. (2002) Early protection against sudden death by $n-3$ polyunsaturated fatty acids after myocardial infarction: time-course analysis of the results of the Gruppo Italiano per lo Studio della Sopravvivenza nell'Infarto Miocardico (GISSI)-Prevenzione. Circulation 105, $1897-1903$

177. Bucher HC, Hengstler P, Schindler C, et al. (2002) n-3 polyunsaturated fatty acids in coronary heart disease: a metaanalysis of randomized controlled trials. Am J Med 112, $298-304$.
178. Studer M, Briel M, Leimenstoll B, et al. (2005) Effect of different antilipidemic agents and diets on mortality: a systematic review. Arch Intern Med 165, 725-730.

179. World Health Organisation (2006) Obesity http://www.who. int/dietphysicalactivity/publications/facts/obesity/en/2006

180. Hutley L \& Prins JB (2005) Fat as an endocrine organ: relationship to the metabolic syndrome. Am J Med Sci 330, 280-289.

181. Fantuzzi G (2005) Adipose tissue, adipokines, and inflammation. J Allergy Clin Immunol 115, 911-919.

182. Halaas JL, Gajiwala KS, Maffei M, et al. (1995) Weightreducing effects of the plasma protein encoded by the obese gene. Science 269, 543-546.

183. Montague CT, Farooqi IS, Whitehead JP, et al. (1997) Congenital leptin deficiency is associated with severe early-onset obesity in humans. Nature 387, 903-908.

184. Greenberg AS \& Obin MS (2006) Obesity and the role of adipose tissue in inflammation and metabolism. Am J Clin Nutr 83, 461S-465S.

185. Hotamisligil GS, Peraldi P, Budavari A, et al. (1996) IRS-1mediated inhibition of insulin receptor tyrosine kinase activity in TNF-alpha- and obesity-induced insulin resistance. Science 271, 665-668.

186. Coppack SW (2001) Pro-inflammatory cytokines and adipose tissue. Proc Nutr Soc 60, 349-356.

187. Gil-Campos M, Cañete RR \& Gil A (2004) Adiponectin, the missing link in insulin resistance and obesity. Clin Nutr 23, 963-974

188. Hausman DB, DiGirolamo M, Bartness TJ, et al. (2001) The biology of white adipocyte proliferation. Obes Rev 2, 239-254.

189. Neels JG \& Olefsky JM (2006) Inflamed fat: what starts the fire? J Clin Invest 116, 33-35.

190. Unger RH (2003) Lipid overload and overflow: metabolic trauma and the metabolic syndrome. Trends Endocrinol Metab 14, 398-403.

191. Unger RH (2003) Minireview: weapons of lean body mass destruction: the role of ectopic lipids in the metabolic syndrome. Endocrinology 144, 5159-5165.

192. McGarry JD (2002) Banting lecture 2001: dysregulation of fatty acid metabolism in the etiology of type 2 diabetes. Diabetes 51, 7-18.

193. Alessi MC, Lijnen HR, Bastelica D, et al. (2003) Adipose tissue and atherothrombosis. Pathophysiol Haemost Thromb 33, 290-297.

194. Bougoulia M, Triantos A \& Koliakos G (2006) Effect of weight loss with or without orlistat treatment on adipocytokines, inflammation, and oxidative markers in obese women. Hormones (Athens) 5, 259-269.

195. Cancello R \& Clement K (2006) Is obesity an inflammatory illness? Role of low-grade inflammation and macrophage infiltration in human white adipose tissue. BJOG 113, 1141-1147.

196. Zagorski SM, Papa NN \& Chung MH (2005) The effect of weight loss after gastric bypass on C-reactive protein levels. Surg Obes Relat Dis 1, 81-85.

197. Reinehr T, Stoffel-Wagner B, Roth CL, et al. (2005) Highsensitive C-reactive protein, tumor necrosis factor alpha, and cardiovascular risk factors before and after weight loss in obese children. Metabolism 54, 1155-1161.

198. Hansen EN, Torquati A \& Abumrad NN (2006) Results of bariatric surgery. Annu Rev Nutr 26, 481-511.

199. Knowler WC, Barrett-Connor E, Fowler SE, et al. (2002) Reduction in the incidence of type 2 diabetes with lifestyle intervention or metformin. N Engl J Med 346, 393-403.

200. Tuomilehto J, Lindstrom J, Eriksson JG, et al. (2001) Prevention of type 2 diabetes mellitus by changes in lifestyle among subjects with impaired glucose tolerance. N Engl J Med 344, $1343-1350$. 
201. Calder PC \& Burdge GC (2004) Fatty acids. In Bioactive Lipids, pp. 1-36 [A Nicolaou and G Kokotos, editors]. Bridgewater: The Oily Press.

202. Calder PC (2001) n-3 Polyunsaturated fatty acids, inflammation and immunity: pouring oil on troubled waters or another fishy tale? Nutr Rev 21, 309-341.

203. Hulshof KF, van Erp-Baart MA, Anttolainen M, et al. (1999) Intake of fatty acids in western Europe with emphasis on trans fatty acids: the TRANSFAIR Study. Eur J Clin Nutr 53, $143-157$.

204. Burdge GC \& Calder PC (2005) Conversion of alpha-linolenic acid to longer-chain polyunsaturated fatty acids in human adults. Reprod Nutr Dev 45, 581-597.

205. Frayn K (2003) Metabolic Regulation: A Human Perspective. Oxford: Blackwell Science.

206. Lewis RA, Austen KF \& Soberman RJ (1990) Leukotrienes and other products of the 5-lipoxygenase pathway. Biochemistry and relation to pathobiology in human diseases. $N$ Engl $J$ Med 323, 645-655.

207. Tilley SL, Coffman TM \& Koller BH (2001) Mixed messages: modulation of inflammation and immune responses by prostaglandins and thromboxanes. J Clin Invest 108, 15-23.

208. Kelley DS, Taylor PC, Nelson GJ, et al. (1998) Arachidonic acid supplementation enhances synthesis of eicosanoids without suppressing immune functions in young healthy men. Lipids 33, 125-130.

209. Thies F, Miles EA, Nebe-von-Caron G, et al. (2001) Influence of dietary supplementation with long-chain $n-3$ or $n-6$ polyunsaturated fatty acids on blood inflammatory cell populations and functions and on plasma soluble adhesion molecules in healthy adults. Lipids 36, 1183-1193.

210. Healy DA, Wallace FA, Miles EA, et al. (2000) Effect of lowto-moderate amounts of dietary fish oil on neutrophil lipid composition and function. Lipids 35, 763-768.

211. Rees D, Miles EA, Banerjee T, et al. (2006) Dose-related effects of eicosapentaenoic acid on innate immune function in healthy humans: a comparison of young and older men. Am J Clin Nutr 83, 331-342.

212. Kelley DS, Taylor PC, Nelson GJ, et al. (1999) Docosahexaenoic acid ingestion inhibits natural killer cell activity and production of inflammatory mediators in young healthy men. Lipids 34, 317-324.

213. Serhan CN, Arita M, Hong S, et al. (2004) Resolvins, docosatrienes, and neuroprotectins, novel omega-3-derived mediators, and their endogenous aspirin-triggered epimers. Lipids 39, $1125-1132$.

214. Serhan CN (2005) Novel eicosanoid and docosanoid mediators: resolvins, docosatrienes, and neuroprotectins. Curr Opin Clin Nutr Metab Care 8, 115-121.

215. Hughes DA, Pinder AC, Piper Z, et al. (1996) Fish oil supplementation inhibits the expression of major histocompatibility complex class II molecules and adhesion molecules on human monocytes. Am J Clin Nutr 63, 267-272.

216. Miles EA, Thies F, Wallace FA, et al. (2001) Influence of age and dietary fish oil on plasma soluble adhesion molecule concentrations. Clin Sci 100, 91-100.

217. Caughey GE, Mantzioris E, Gibson RA, et al. (1996) The effect on human tumor necrosis factor alpha and interleukin 1 beta production of diets enriched in n-3 fatty acids from vegetable oil or fish oil. Am J Clin Nutr 63, 116-122.

218. Mori TA, Woodman RJ, Burke V, et al. (2003) Effect of eicosapentaenoic acid and docosahexaenoic acid on oxidative stress and inflammatory markers in treated-hypertensive type 2 diabetic subjects. Free Radic Biol Med 35, 772-781.

219. Burdge GC \& Calder PC (2006) Dietary $\alpha$-linolenic acid and health-related outcomes: a metabolic perspective. Nutr Res Rev 19, 26-52.
220. Lopez-Garcia E, Schulze MB, Fung TT, et al. (2004) Major dietary patterns are related to plasma concentrations of markers of inflammation and endothelial dysfunction. Am J Clin Nutr 80, $1029-1035$.

221. Rallidis LS, Paschos G, Papaioannou ML, et al. (2004) The effect of diet enriched with alpha-linolenic acid on soluble cellular adhesion molecules in dyslipidaemic patients Atherosclerosis 174, 127-132.

222. Bemelmans WJ, Lefrandt JD, Feskens EJ, et al. (2004) Increased alpha-linolenic acid intake lowers $\mathrm{C}$-reactive protein, but has no effect on markers of atherosclerosis. Eur J Clin Nutr 58, $1083-1089$.

223. Rallidis LS, Paschos G, Liakos GK, et al. (2003) Dietary alpha-linolenic acid decreases C-reactive protein, serum amyloid A and interleukin-6 in dyslipidaemic patients. Atherosclerosis 167, 237-242.

224. Zhao G, Etherton TD, Martin KR, et al. (2004) Dietary alphalinolenic acid reduces inflammatory and lipid cardiovascular risk factors in hypercholesterolemic men and women. $J$ Nutr 134, 2991-2997.

225. Arita M, Yoshida M, Hong S, et al. (2005) Resolvin E1, an endogenous lipid mediator derived from omega-3 eicosapentaenoic acid, protects against 2,4,6-trinitrobenzene sulfonic acid-induced colitis. Proc Natl Acad Sci U S A 102, 7671-7676.

226. Calder PC (2003) n-3 Polyunsaturated fatty acids and inflammation: from molecular biology to the clinic. Lipids 38, $342-352$.

227. Calder PC (2004) n-3 fatty acids, inflammation, and immunity relevance to postsurgical and critically ill patients. Lipids $\mathbf{3 9}$, $1147-1161$.

228. Lee JY, Plakidas A, Lee WH, et al. (2003) Differential modulation of Toll-like receptors by fatty acids: preferential inhibition by $n-3$ polyunsaturated fatty acids. J Lipid Res 44, 479-486.

229. Lee JY, Ye J, Gao Z, et al. (2003) Reciprocal modulation of Toll-like receptor-4 signaling pathways involving MyD88 and phosphatidylinositol 3-kinase/AKT by saturated and polyunsaturated fatty acids. $J$ Biol Chem 278, 37041-37051.

230. Lee JY, Zhao L, Youn HS, et al. (2004) Saturated fatty acid activates but polyunsaturated fatty acid inhibits Toll-like receptor 2 dimerized with Toll-like receptor 6 or $1 . \mathrm{J}$ Biol Chem 279, 16971-16979.

231. Weatherill AR, Lee JY, Zhao L, et al. (2005) Saturated and polyunsaturated fatty acids reciprocally modulate dendritic cell functions mediated through TLR4. J Immunol 174, $5390-5397$

232. Shoda R, Matsueda K, Yamato S, et al. (1996) Epidemiologic analysis of Crohn disease in Japan: increased dietary intake of $\mathrm{n}-6$ polyunsaturated fatty acids and animal protein relates to the increased incidence of Crohn disease in Japan. Am J Clin Nutr 63, 741-745.

233. Belluzzi A, Brignola C, Campieri M, et al. (1996) Effect of an enteric-coated fish-oil preparation on relapses in Crohn's disease. $N$ Engl J Med 334, 1557-1560.

234. MacLean CH, Mojica WA, Morton SC, et al. (2004) Effects of Omega-3 Fatty Acids on Inflammatory Bowel Disease, Rheumatoid Arthritis, Renal Disease, Systemic Lupus Erythematosus, and Osteoporosis. Rockville, IN: Agency for Healthcare Research and Quality.

235. Dunstan JA, Mori TA, Barden A, et al. (2003) Maternal fish oil supplementation in pregnancy reduces interleukin-13 levels in cord blood of infants at high risk of atopy. Clin Exp Allergy 33 $442-448$.

236. Dunstan JA, Mori TA, Barden A, et al. (2003) Fish oil supplementation in pregnancy modifies neonatal allergen-specific immune responses and clinical outcomes in infants at high 
risk of atopy: a randomized, controlled trial. J Allergy Clin Immunol 112, 1178-1184.

237. Thien FCK, Woods R, De Luca S, et al. (2002) Dietary marine fatty acids (fish oil) for asthma in adults and children, Cochrane Database of Systematic Reviews, CD001283.

238. Nagakura T, Matsuda S, Shichijyo K, et al. (2000) Dietary supplementation with fish oil rich in omega-3 polyunsaturated fatty acids in children with bronchial asthma. Eur Respir J 16, $861-865$.

239. Schachter H, Reisman J, Tran K, et al. (2004) Health Effects of Omega-3 Fatty Acids on Asthma. Rockville, IN: Agency for Healthcare and Quality.

240. Broughton KS, Johnson CS, Pace BK, et al. (1997) Reduced asthma symptoms with $n$-3 fatty acid ingestion are related to 5-series leukotriene production. Am J Clin Nutr 65, 1011-1017.

241. Broekhuizen R, Wouters EF, Creutzberg EC, et al. (2005) Polyunsaturated fatty acids improve exercise capacity in chronic obstructive pulmonary disease. Thorax 60, 376-382.

242. Matsuyama W, Mitsuyama H, Watanabe M, et al. (2005) Effects of omega-3 polyunsaturated fatty acids on inflammatory markers in COPD. Chest 128, 3817-3827.

243. Adam O, Beringer C, Kless T, et al. (2003) Anti-inflammatory effects of a low arachidonic acid diet and fish oil in patients with rheumatoid arthritis. Rheumatol Int 23, 27-36.

244. Fortin PR, Lew RA, Liang MH, et al. (1995) Validation of a meta-analysis: the effects of fish oil in rheumatoid arthritis. $J$ Clin Epidemiol 48, 1379-1390.

245. Mayser P, Mrowietz U, Arenberger P, et al. (1998) Omega-3 fatty acid-based lipid infusion in patients with chronic plaque psoriasis: results of a double-blind, randomized, placebocontrolled, multicenter trial. J Am Acad Dermatol 38, 539-547.

246. Ziboh V (1998) The role of $n-3$ fatty acids in psoriasis. In Medicinal Fatty Acids in Inflammation, pp. 45-53 [J Kremer, editor]. Basel: Birkhauser Verlag.

247. Rhodes LE, O'Farrell S, Jackson MJ, et al. (1994) Dietary fish oil supplementation in humans reduces UVB-erythemal sensitivity but increases epidermal lipid peroxidation. $J$ Invest Dermatol 103, 151-154.

248. Rhodes LE, Durham B, Fraser W, et al. (1994) Fish oil reduces PGE2 levels in skin and increases the threshold to provocation of polymorphic light eruption. J Invest Dermatol 103, 404.

249. Kris-Etherton PM, Harris WS \& Appel LJ (2002) Fish consumption, fish oil, omega-3 fatty acids, and cardiovascular disease. Circulation 106, 2747-2757.

250. Calder PC (2004) $n$-3 Fatty acids and cardiovascular disease: evidence explained and mechanisms explored. Clin Sci 107, 1-11.

251. Serafini M \& Del RD (2004) Understanding the association between dietary antioxidants, redox status and disease: is the Total Antioxidant Capacity the right tool? Redox Rep 9, $145-152$.

252. Serafini M, Bugianesi R, Maiani G, et al. (2003) Plasma antioxidants from chocolate. Nature 424, 1013.

253. Vassalle C, Petrozzi L, Botto N, et al. (2004) Oxidative stress and its association with coronary artery disease and different atherogenic risk factors. J Intern Med 256, 308-315.

254. Ching S, Ingram D, Hahnel R, et al. (2002) Serum levels of micronutrients, antioxidants and total antioxidant status predict risk of breast cancer in a case control study. $J$ Nutr $\mathbf{1 3 2}$ 303-306.

255. Serafini M, Bellocco R, Wolk A, et al. (2002) Total antioxidant potential of fruit and vegetables and risk of gastric cancer. Gastroenterology 123, 985-991.

256. Agudo A, Cabrera L, Amiano P, et al. (2007) Fruit and vegetable intakes, dietary antioxidant nutrients, and total mortality in Spanish adults: findings from the Spanish cohort of the European Prospective Investigation into Cancer and Nutrition (EPIC-Spain). Am J Clin Nutr 85, 1634-1642.
257. Brighenti F, Valtuena S, Pellegrini N, et al. (2005) Total antioxidant capacity of the diet is inversely and independently related to plasma concentration of high-sensitivity C-reactive protein in adult Italian subjects. Br J Nutr 93, 619-625.

258. Food and Nutrition Board IoM (2000) Dietary Reference Intakes for Vitamin C, Vitamin E, Selenium and Carotenoids. Washington.

259. Levine M, Conry-Cantilena C, Wang Y, et al. (1996) Vitamin $\mathrm{C}$ pharmacokinetics in healthy volunteers: evidence for a recommended dietary allowance. Proc Natl Acad Sci U S A 93, 3704-3709.

260. Frei B, England L \& Ames BN (1989) Ascorbate is an outstanding antioxidant in human blood plasma. Proc Natl Acad Sci U S A 86, 6377-6381.

261. Levine M, Dhariwal KR, Wang Y, et al. (1994) Ascorbic acid in neutrophils. In Natural Antioxidants in Health and Disease, pp. 469-488 [B Frei, editor]. San Diego, CA: Academic Press.

262. Frei B, Stocker R \& Ames BN (1988) Antioxidant defenses and lipid peroxidation in human blood plasma. Proc Natl Acad Sci U S A 85, 9748-9752.

263. Jialal I, Vega GL \& Grundy SM (1990) Physiologic levels of ascorbate inhibit the oxidative modification of low density lipoprotein. Atherosclerosis 82, 185-191.

264. Halpner AD, Handelman GJ, Belmont CA, et al. (1998) Protection by vitamin $\mathrm{C}$ of oxidant-induced loss of vitamin $\mathrm{E}$ in rat hepatocytes. $J$ Nutr Biochem 9, 355-359.

265. Horrobin DF (1996) Ascorbic acid and prostaglandin synthesis. Subcell Biochem 25, 109-115.

266. Halliwell B, Wasil M \& Grootveld M (1987) Biologically significant scavenging of the myeloperoxidase-derived oxidant hypochlorous acid by ascorbic acid. Implications for antioxidant protection in the inflamed rheumatoid joint. FEBS Lett 213, 15-17.

267. Anderson R \& Lukey PT (1987) A biological role for ascorbate in the selective neutralization of extracellular phagocyte-derived oxidants. Ann N Y Acad Sci 498, 229-247.

268. Goetzl EJ, Wasserman SI, Gigli I, et al. (1974) Enhancement of random migration and chemotactic response of human leukocytes by ascorbic acid. J Clin Invest 53, 813-818.

269. Sanchez-Moreno C, Cano MP, de Ancos B, et al. (2004) Consumption of high-pressurized vegetable soup increases plasma vitamin $\mathrm{C}$ and decreases oxidative stress and inflammatory biomarkers in healthy humans. J Nutr 134, 3021-3025.

270. Sanchez-Moreno C, Dashe JF, Scott T, et al. (2004) Decreased levels of plasma vitamin $\mathrm{C}$ and increased concentrations of inflammatory and oxidative stress markers after stroke. Stroke 35, $163-168$

271. Evans RM, Currie L \& Campbell A (1982) The distribution of ascorbic acid between various cellular components of blood, in normal individuals, and its relation to the plasma concentration. Br J Nutr 47, 473-482.

272. Lunec J \& Blake DR (1985) The determination of dehydroascorbic acid and ascorbic acid in the serum and synovial fluid of patients with rheumatoid arthritis (RA). Free Radic Res Commun 1, 31-39.

273. Cross CE, Forte T, Stocker R, et al. (1990) Oxidative stress and abnormal cholesterol metabolism in patients with adult respiratory distress syndrome. J Lab Clin Med 115, 396-404.

274. Imes S, Dinwoodie A, Walker K, et al. (1986) Vitamin C status in 137 outpatients with Crohn's disease. Effect of diet counseling. J Clin Gastroenterol 8, 443-446.

275. Hoffenberg EJ, Deutsch J, Smith S, et al. (1997) Circulating antioxidant concentrations in children with inflammatory bowel disease. Am J Clin Nutr 65, 1482-1488.

276. Filippi J, Al-Jaouni R, Wiroth JB, et al. (2006) Nutritional deficiencies in patients with Crohn's disease in remission. Inflamm Bowel Dis 12, 185-191. 
277. Wendland BE, Aghdassi E, Tam C, et al. (2001) Lipid peroxidation and plasma antioxidant micronutrients in Crohn disease. Am J Clin Nutr 74, 259-264.

278. Buffinton GD \& Doe WF (1995) Altered ascorbic acid status in the mucosa from inflammatory bowel disease patients. Free Radic Res 22, 131-143.

279. Aghdassi E, Wendland BE, Steinhart AH, et al. (2003) Antioxidant vitamin supplementation in Crohn's disease decreases oxidative stress. a randomized controlled trial. Am J Gastroenterol 98, 348-353.

280. Trebble TM, Arden NK, Wootton SA, et al. (2004) Fish oil and antioxidants alter the composition and function of circulating mononuclear cells in Crohn disease. Am J Clin Nutr 80, $1137-1144$.

281. Kelly FJ, Mudway I, Blomberg A, et al. (1999) Altered lung antioxidant status in patients with mild asthma. Lancet 354, $482-483$.

282. Winklhofer-Roob BM, Ellemunter H, Fruhwirth M, et al. (1997) Plasma vitamin C concentrations in patients with cystic fibrosis: evidence of associations with lung inflammation. Am J Clin Nutr 65, 1858-1866.

283. Schwartz J \& Weiss ST (1994) Relationship between dietary vitamin $\mathrm{C}$ intake and pulmonary function in the First National Health and Nutrition Examination Survey (NHANES I). Am J Clin Nutr 59, 110-114.

284. Britton JR, Pavord ID, Richards KA, et al. (1995) Dietary antioxidant vitamin intake and lung function in the general population. Am J Respir Crit Care Med 151, 1383-1387.

285. Harik-Khan RI, Muller DC \& Wise RA (2004) Serum vitamin levels and the risk of asthma in children. Am J Epidemiol 159, 351-357.

286. Devereux G, Turner SW, Craig LC, et al. (2006) Low maternal vitamin $\mathrm{E}$ intake during pregnancy is associated with asthma in 5-year-old children. Am J Respir Crit Care Med 174, 499-507.

287. Aderele WI, Ette SI, Oduwole O, et al. (1985) Plasma vitamin $\mathrm{C}$ (ascorbic acid) levels in asthmatic children. Afr J Med Med Sci 14, 115-120.

288. Bucca C, Rolla G \& Farina JC (1992) Effect of vitamin C on transient increase of bronchial responsiveness in conditions affecting the airways. Ann N Y Acad Sci 669, 175-186.

289. Cohen HA, Neuman I \& Nahum H (1997) Blocking effect of vitamin $\mathrm{C}$ in exercise-induced asthma. Arch Pediatr Adolesc Med 151, 367-370.

290. Tecklenburg SL, Mickleborough TD, Fly AD, et al. (2007) Ascorbic acid supplementation attenuates exercise-induced bronchoconstriction in patients with asthma. Respir Med 101, $1770-1778$.

291. Hagfors L, Leanderson P, Skoldstam L, et al. (2003) Antioxidant intake, plasma antioxidants and oxidative stress in a randomized, controlled, parallel, Mediterranean dietary intervention study on patients with rheumatoid arthritis. Nutr $J$ 2,5 .

292. Traber MG (2007) Heart disease and single-vitamin supplementation. Am J Clin Nutr 85, 293S-299S.

293. van Herpen-Broekmans WM, Klopping-Ketelaars IA, Bots ML, et al. (2004) Serum carotenoids and vitamins in relation to markers of endothelial function and inflammation. Eur $J$ Epidemiol 19, 915-921.

294. Plantinga Y, Ghiadoni L, Magagna A, et al. (2007) Supplementation with vitamins $\mathrm{C}$ and $\mathrm{E}$ improves arterial stiffness and endothelial function in essential hypertensive patients. Am J Hypertens 20, 392-397.

295. Tahir M, Foley B, Pate G, et al. (2005) Impact of vitamin E and $\mathrm{C}$ supplementation on serum adhesion molecules in chronic degenerative aortic stenosis: a randomized controlled trial. Am Heart J 150, 302-306.
296. Traber MG, Burton GW, Ingold KU, et al. (1990) RRR- and SRR-alpha-tocopherols are secreted without discrimination in human chylomicrons, but RRR-alpha-tocopherol is preferentially secreted in very low density lipoproteins. $J$ Lipid Res 31, 675-685.

297. Schultz M, Leist M, Elsner A, et al. (1997) Alpha-carboxyethyl-6-hydroxychroman as urinary metabolite of vitamin E Methods Enzymol 282, 297-310.

298. Lodge JK, Ridlington J, Leonard S, et al. (2001) Alpha- and gamma-tocotrienols are metabolized to carboxyethyl-hydroxychroman derivatives and excreted in human urine. Lipids 36, $43-48$.

299. McLaughlin PJ \& Weihrauch JL (1979) Vitamin E content of foods. J Am Diet Assoc 75, 647-665.

300. Lehmann J, Martin HL, Lashley EL, et al. (1986) Vitamin E in foods from high and low linoleic acid diets. J Am Diet Assoc 86, $1208-1216$.

301. Murphy SP, Subar AF \& Block G (1990) Vitamin E intakes and sources in the United States. Am J Clin Nutr 52, 361-367.

302. Slesinski MJ, Subar AF \& Kahle LL (1996) Dietary intake of fat, fiber and other nutrients is related to the use of vitamin and mineral supplements in the United States: the 1992 National Health Interview Survey. J Nutr 126, 3001-3008.

303. Burton GW \& Ingold KU (1986) Vitamin E: Application of the principles of physical organic chemistry to the exploration of its structure and functions. Acc Chem Rec 19, 194-201.

304. Kamal-Eldin A \& Appelqvist LA (1996) The chemistry and antioxidant properties of tocopherols and tocotrienols. Lipids 31, 671-701.

305. Jiang Q, Christen S, Shigenaga MK, et al. (2001) Gammatocopherol, the major form of vitamin $\mathrm{E}$ in the US diet, deserves more attention. Am J Clin Nutr 74, 714-722.

306. Winklhofer-Roob BM, Rock E, Ribalta J, et al. (2003) Effects of vitamin $\mathrm{E}$ and carotenoid status on oxidative stress in health and disease. Evidence obtained from human intervention studies. Mol Aspects Med 24, 391-402.

307. Winklhofer-Roob BM, Ziouzenkova O, Puhl H, et al. (1995) Impaired resistance to oxidation of low density lipoprotein in cystic fibrosis: improvement during vitamin E supplementation. Free Radic Biol Med 19, 725-733.

308. Saldeen T, Li D \& Mehta JL (1999) Differential effects of alpha- and gamma-tocopherol on low-density lipoprotein oxidation, superoxide activity, platelet aggregation and arterial thrombogenesis. J Am Coll Cardiol 34, 1208-1215.

309. Liu M, Wallin R, Wallmon A, et al. (2002) Mixed tocopherols have a stronger inhibitory effect on lipid peroxidation than alpha-tocopherol alone. J Cardiovasc Pharmacol 39, $714-721$.

310. Devaraj S, Hugou I \& Jialal I (2001) Alpha-tocopherol decreases CD36 expression in human monocyte-derived macrophages. J Lipid Res 42, 521-527.

311. Bieri JG \& Evarts RP (1974) Vitamin E activity of gammatocopherol in the rat, chick and hamster. J Nutr 104, 850-857.

312. Cooney RV, Franke AA, Harwood PJ, et al. (1993) Gammatocopherol detoxification of nitrogen dioxide: superiority to alpha-tocopherol. Proc Natl Acad Sci U S A 90, 1771-1775.

313. Christen S, Woodall AA, Shigenaga MK, et al. (1997) Gamma-tocopherol traps mutagenic electrophiles such as $\mathrm{NO}(\mathrm{X})$ and complements alpha-tocopherol: physiological implications. Proc Natl Acad Sci U S A 94, 3217-3222.

314. Devaraj S \& Jialal I (2000) Alpha tocopherol supplementation decreases serum C-reactive protein and monocyte interleukin-6 levels in normal volunteers and type 2 diabetic patients. Free Radic Biol Med 29, 790-792.

315. Sundl I, Gruber HJ \& Roob JM (2005) Effects of mixed tocopherols versus alpha-tocopherol on nuclear factor kappa 
B activation in mononuclear leukocytes of healthy male volunteers. Clin Nutr 24, 595.

316. Tasinato A, Boscoboinik D, Bartoli GM, et al. (1995) D-alphatocopherol inhibition of vascular smooth muscle cell proliferation occurs at physiological concentrations, correlates with protein kinase $\mathrm{C}$ inhibition, and is independent of its antioxidant properties. Proc Natl Acad Sci U S A 92, 12190-12194.

317. Devaraj S \& Jialal I (1999) Alpha-tocopherol decreases interleukin- 1 beta release from activated human monocytes by inhibition of 5-lipoxygenase. Arterioscler Thromb Vasc Biol 19, $1125-1133$.

318. Jiang Q, Lykkesfeldt J, Shigenaga MK, et al. (2002) Gammatocopherol supplementation inhibits protein nitration and ascorbate oxidation in rats with inflammation. Free Radic Biol Med 33, 1534-1542.

319. Grammas P, Hamdheydari L, Benaksas EJ, et al. (2004) Anti-inflammatory effects of tocopherol metabolites. Biochem Biophys Res Commun 319, 1047-1052.

320. Jiang Q \& Ames BN (2003) Gamma-tocopherol, but not alpha-tocopherol, decreases proinflammatory eicosanoids and inflammation damage in rats. FASEB $J$ 17, 816-822.

321. Li D, Saldeen T \& Mehta JL (1999) Gamma-tocopherol decreases ox-LDL-mediated activation of nuclear factorkappaB and apoptosis in human coronary artery endothelial cells. Biochem Biophys Res Commun 259, 157-161.

322. Jiang Q, Elson-Schwab I, Courtemanche C, et al. (2000) Gamma-tocopherol and its major metabolite, in contrast to alpha-tocopherol, inhibit cyclooxygenase activity in macrophages and epithelial cells. Proc Natl Acad Sci U S A 97, 11494-11499.

323. Heliovaara M, Knekt P, Aho K, et al. (1994) Serum antioxidants and risk of rheumatoid arthritis. Ann Rheum Dis 53, 51-53.

324. De Bandt M, Grossin M, Driss F, et al. (2002) Vitamin E uncouples joint destruction and clinical inflammation in a transgenetic mouse model of rheumatoid arthritis. Arthritis Rheum 46, 522-532.

325. Venkatraman JT \& Chu WC (1999) Effects of dietary omega-3 and omega- 6 lipids and vitamin $\mathrm{E}$ on serum cytokines, lipid mediators and anti-DNA antibodies in a mouse model for rheumatoid arthritis. J Am Coll Nutr 18, 602-613.

326. Edmonds SE, Winyard PG, Guo R, et al. (1997) Putative analgesic activity of repeated oral doses of vitamin $\mathrm{E}$ in the treatment of rheumatoid arthritis. Results of a prospective placebo controlled double blind trial. Ann Rheum Dis 56, 649-655.

327. Dieber-Rotheneder M, Puhl H, Waeg G, et al. (1991) Effect of oral supplementation with D-alpha-tocopherol on the vitamin $\mathrm{E}$ content of human low density lipoproteins and resistance to oxidation. J Lipid Res 32, 1325-1332.

328. Steinbrecher UP, Parthasarathy S, Leake DS, et al. (1984) Modification of low density lipoprotein by endothelial cells involves lipid peroxidation and degradation of low density lipoprotein phospholipids. Proc Natl Acad Sci U S A 81, 3883-3887.

329. Azzi A, Boscoboinik D \& Marilley D (1995) Vitamin E: a sensor and an information transducer of the cell oxidation state. Am J Clin Nutr 62, 1337S-1346S.

330. Freedman JE, Farhat JH, Loscalzo J, et al. (1996) Alphatocopherol inhibits aggregation of human platelets by a protein kinase C-dependent mechanism. Circulation 94, 2434-2440.

331. Devaraj S, Li D \& Jialal I (1996) The effects of alpha tocopherol supplementation on monocyte function. Decreased lipid oxidation, interleukin 1 beta secretion, and monocyte adhesion to endothelium. J Clin Invest 98, 756-763.
332. Chan AC \& Leith MK (1981) Decreased prostacyclin synthesis in vitamin E-deficient rabbit aorta. Am J Clin Nutr 34, 2341-2347.

333. Tran K \& Chan AC (1990) R,R,R-alpha-tocopherol potentiates prostacyclin release in human endothelial cells. Evidence for structural specificity of the tocopherol molecule. Biochim Biophys Acta 1043, 189-197.

334. Chan AC, Wagner M, Kennedy C, et al. (1998) Vitamin E up-regulates arachidonic acid release and phospholipase A2 in megakaryocytes. Mol Cell Biochem 189, 153-159.

335. Cominacini L, Garbin U, Pasini AF, et al. (1997) Antioxidants inhibit the expression of intercellular cell adhesion molecule-1 and vascular cell adhesion molecule-1 induced by oxidized LDL on human umbilical vein endothelial cells. Free Radic Biol Med 22, 117-127.

336. Ohrvall M, Sundlof G \& Vessby B (1996) Gamma, but not alpha, tocopherol levels in serum are reduced in coronary heart disease patients. J Intern Med 239, 111-117.

337. Handelman GJ, Machlin LJ, Fitch K, et al. (1985) Oral alphatocopherol supplements decrease plasma gamma-tocopherol levels in humans. $J$ Nutr 115, 807-813.

338. Sundl I, Resch U, Bergmann AR, et al. (2004) The decrease in gamma-tocopherol in plasma and lipoprotein fractions levels off within two days of vitamin E supplementation. Ann $N Y$ Acad Sci 1031, 378-380.

339. Drewnowski A, Rock CL, Henderson SA, et al. (1997) Serum beta-carotene and vitamin $\mathrm{C}$ as biomarkers of vegetable and fruit intakes in a community-based sample of French adults. Am J Clin Nutr 65, 1796-1802.

340. Krinsky NI (1993) Actions of carotenoids in biological systems. Annu Rev Nutr 13, 561-587.

341. Krinsky NI (2002) Possible biologic mechanisms for a protective role of xanthophylls. J Nutr 132, 540S-542S.

342. Bai SK, Lee SJ, Na HJ, et al. (2005) beta-Carotene inhibits inflammatory gene expression in lipopolysaccharide-stimulated macrophages by suppressing redox-based NF-kappaB activation. Exp Mol Med 37, 323-334.

343. Flohé L, Brigelius-Flohé R, Saliou C, et al. (1997) Redox regulation of NF-kB activation. Free Radic Biol Med 22, 1115-1126.

344. Louw JA, Werbeck A, Louw ME, et al. (1992) Blood vitamin concentrations during the acute-phase response. Crit Care Med 20, 934-941.

345. Kritchevsky SB, Bush AJ, Pahor M, et al. (2000) Serum carotenoids and markers of inflammation in nonsmokers. Am J Epidemiol 152, 1065-1071.

346. Quasim T, McMillan DC, Talwar D, et al. (2003) Lower concentrations of carotenoids in the critically ill patient are related to a systemic inflammatory response and increased lipid peroxidation. Clin Nutr 22, 459-462.

347. Lakshman MR \& Rao MN (1999) Purification and characterization of cellular carotenoid-binding protein from mammalian liver. Methods Enzymol 299, 441-456.

348. Kim GY, Kim JH, Ahn SC, et al. (2004) Lycopene suppresses the lipopolysaccharide-induced phenotypic and functional maturation of murine dendritic cells through inhibition of mitogen-activated protein kinases and nuclear factor-kappaB. Immunology 113, 203-211.

349. Ohgami K, Shiratori K, Kotake S, et al. (2003) Effects of astaxanthin on lipopolysaccharide-induced inflammation in vitro and in vivo. Invest Ophthalmol Vis Sci 44, 2694-2701.

350. Lee EH, Faulhaber D, Hanson KM, et al. (2004) Dietary lutein reduces ultraviolet radiation-induced inflammation and immunosuppression. J Invest Dermatol 122, 510-517.

351. Watzl B, Kulling SE, Möseneder J, et al. (2005) A 4-week intervention with high intake of carotenoid-rich vegetables 
and fruit reduces plasma C-reactive protein in healthy, nonsmoking men. Am J Clin Nutr 82, 1052-1058.

352. Riso P, Visioli F, Grande S, et al. (2006) Effect of a tomatobased drink on markers of inflammation, immunomodulation, and oxidative stress. J Agric Food Chem 54, 2563-2566.

353. Rumi G Jr, Szabo I, Vincze A, et al. (2000) Decrease of serum carotenoids in Crohn's disease. J Physiol Paris 94, 159-161.

354. Reifen R, Nur T, Matas Z, et al. (2001) Lycopene supplementation attenuates the inflammatory status of colitis in a rat model. Int J Vitam Nutr Res 71, 347-351.

355. Wood LG, Garg ML, Blake RJ, et al. (2004) Carotenoid concentrations in asthmatics versus healthy controls. Asia Pac J Clin Nutr 13, S74.

356. Cerhan JR, Saag KG, Merlino LA, et al. (2003) Antioxidant micronutrients and risk of rheumatoid arthritis in a cohort of older women. Am J Epidemiol 157, 345-354.

357. Pattison DJ, Symmons DP, Lunt M, et al. (2005) Dietary betacryptoxanthin and inflammatory polyarthritis: results from a population-based prospective study. Am J Clin Nutr 82, 451-455.

358. Comstock GW, Burke AE, Hoffman SC, et al. (1997) Serum concentrations of alpha tocopherol, beta carotene, and retinol preceding the diagnosis of rheumatoid arthritis and systemic lupus erythematosus. Ann Rheum Dis 56, 323-325.

359. Sesso HD, Buring JE, Norkus EP, et al. (2004) Plasma lycopene, other carotenoids, and retinol and the risk of cardiovascular disease in women. Am J Clin Nutr 79, 47-53.

360. Sesso HD, Buring JE, Norkus EP, et al. (2005) Plasma lycopene, other carotenoids, and retinol and the risk of cardiovascular disease in men. Am J Clin Nutr 81, 990-997.

361. Suzuki K, Inoue T, Hioki R, et al. (2006) Association of abdominal obesity with decreased serum levels of carotenoids in a healthy Japanese population. Clin Nutr 25, 780-789.

362. Wallstrom P, Wirfalt E, Lahmann PH, et al. (2001) Serum concentrations of beta-carotene and alpha-tocopherol are associated with diet, smoking, and general and central adiposity. Am J Clin Nutr 73, 777-785.

363. Winklhofer-Roob BM, Sargsyan K \& Maritschnegg M (2005) Relations between antioxidants and biomarkers of consumption of fruits and vegetables and body mass index in the STYrian Juvenile Obesity Study (STYJOBS). Int J Obes 29, S150.

364. Manach C, Scalbert A, Morand C, et al. (2004) Polyphenols: food sources and bioavailability. Am J Clin Nutr 79, 727-747.

365. Kuhnau J (1976) The flavonoids. A class of semi-essential food components: their role in human nutrition. World Rev Nutr Diet 24, 117-191.

366. Baumann J, von Bruchhausen F \& Wurm G (1980) Flavonoids and related compounds as inhibition of arachidonic acid peroxidation. Prostaglandins 20, 627-639.

367. Lee TP, Matteliano ML \& Middleton E Jr (1982) Effect of quercetin on human polymorphonuclear leukocyte lysosomal enzyme release and phospholipid metabolism. Life Sci 31, 2765-2774.

368. Welton AF, Tobias LD, Fiedler-Nagy C, et al. (1986) Effect of flavonoids on arachidonic acid metabolism. Prog Clin Biol Res 213, 231-242.

369. Noreen Y, Serrano G, Perera P, et al. (1998) Flavan-3-ols isolated from some medicinal plants inhibiting COX-1 and COX2 catalysed prostaglandin biosynthesis. Planta Med 64, $520-524$.

370. Chiesi M \& Schwaller R (1995) Inhibition of constitutive endothelial NO-synthase activity by tannin and quercetin. Biochem Pharmacol 49, 495-501.

371. Kim OK, Murakami A, Nakamura Y, et al. (1998) Screening of edible Japanese plants for nitric oxide generation inhibitory activities in RAW 264.7 cells. Cancer Lett 125, 199-207.
372. Liang YC, Huang YT, Tsai SH, et al. (1999) Suppression of inducible cyclooxygenase and inducible nitric oxide synthase by apigenin and related flavonoids in mouse macrophages. Carcinogenesis 20, 1945-1952.

373. Kim HK, Cheon BS, Kim YH, et al. (1999) Effects of naturally occurring flavonoids on nitric oxide production in the macrophage cell line RAW 264.7 and their structure-activity relationships. Biochem Pharmacol 58, 759-765.

374. Geng Y, Zhang B \& Lotz M (1993) Protein tyrosine kinase activation is required for lipopolysaccharide induction of cytokines in human blood monocytes. J Immunol 151, 6692-6700.

375. Cho JY, Kim PS, Park J, et al. (2000) Inhibitor of tumor necrosis factor-alpha production in lipopolysaccharidestimulated RAW264.7 cells from Amorpha fruticosa. J Ethnopharmacol 70, 127-133.

376. Wadsworth TL, McDonald TL \& Koop DR (2001) Effects of Ginkgo biloba extract (EGb 761) and quercetin on lipopolysaccharide-induced signaling pathways involved in the release of tumor necrosis factor-alpha. Biochem Pharmacol 62, 963-974.

377. Wadsworth TL \& Koop DR (2003) Effects of Ginkgo biloba extract (EGb 761) and quercetin on lipopolysaccharideinduced release of nitric oxide. Chem Biol Interact 137, 43-58.

378. Cho SY, Park SJ, Kwon MJ, et al. (2003) Quercetin suppresses proinflammatory cytokines production through MAP kinases and NF-kappaB pathway in lipopolysaccharide-stimulated macrophage. Mol Cell Biochem 243, 153-160.

379. Morikawa K, Nonaka M, Narahara M, et al. (2003) Inhibitory effect of quercetin on carrageenan-induced inflammation in rats. Life Sci 74, 709-721.

380. Takahashi K, Morikawa A, Kato Y, et al. (2001) Flavonoids protect mice from two types of lethal shock induced by endotoxin. FEMS Immunol Med Microbiol 31, 29-33.

381. Ueda H, Yamazaki C \& Yamazaki M (2004) A hydroxyl group of flavonoids affects oral anti-inflammatory activity and inhibition of systemic tumor necrosis factor-alpha production. Biosci Biotechnol Biochem 68, 119-125.

382. Estruch R, Sacanella E, Badia E, et al. (2004) Different effects of red wine and gin consumption on inflammatory biomarkers of atherosclerosis: a prospective randomized crossover trial. Effects of wine on inflammatory markers. Atherosclerosis 175, 117-123.

383. Zern TL, Wood RJ, Greene C, et al. (2005) Grape polyphenols exert a cardioprotective effect in pre- and postmenopausal women by lowering plasma lipids and reducing oxidative stress. J Nutr 135, 1911-1917.

384. Widlansky ME, Duffy SJ, Hamburg NM, et al. (2005) Effects of black tea consumption on plasma catechins and markers of oxidative stress and inflammation in patients with coronary artery disease. Free Radic Biol Med 38, 499-506.

385. Song Y, Manson JE, Buring JE, et al. (2005) Associations of dietary flavonoids with risk of type 2 diabetes, and markers of insulin resistance and systemic inflammation in women: a prospective study and cross-sectional analysis. J Am Coll Nutr 24, 376-384.

386. Bogani P, Galli C, Villa M, et al. (2007) Postprandial antiinflammatory and antioxidant effects of extra virgin olive oil. Atherosclerosis 190, 181-186.

387. Rolfe R (1996) Colonization resistance. In Gastrointentestinal Microbiology, 501-536, , [RI Mackie, BA White and RE Isaacson, editors]. New York: Chapman \& Hall.

388. Holzapfel WH (2006) Introduction to prebiotics and probiotics. In Probiotics in Food Safety and Human Health, pp. 1-33 [I Goktepe, VK Juneja and M Ahmedna, editors]. Boca Raton, FL: CRC Press. 
389. Edwards CA \& Parrett AM (2002) Intestinal flora during the first months of life: new perspectives. Br J Nutr $\mathbf{8 8}$, Suppl. 1, S11-S18.

390. Holzapfel WH, Haberer P, Snel J, et al. (1998) Overview of gut flora and probiotics. Int J Food Microbiol 41, 85-101.

391. Rhee KJ, Sethupathi P, Driks A, et al. (2004) Role of commensal bacteria in development of gut-associated lymphoid tissues and preimmune antibody repertoire. $J$ Immunol 172, $1118-1124$

392. Tanaka K \& Ishikawa H (2004) Role of intestinal bacterial flora in oral tolerance induction. Histol Histopathol 19, 907-914.

393. Yamanaka T, Helgeland L, Farstad IN, et al. (2003) Microbial colonization drives lymphocyte accumulation and differentiation in the follicle-associated epithelium of Peyer's patches. J Immunol 170, 816-822.

394. Wostmann BS (1996) Germfree and Gnotobiotic Animal Models. Boca Raton, FL: CRC Press.

395. Ouwehand A, Isolauri E \& Salminen S (2002) The role of the intestinal microflora for the development of the immune system in early childhood. Eur J Nutr 41, Suppl. 1, I32-I37.

396. Kalliomaki M, Kirjavainen P, Eerola E, et al. (2001) Distinct patterns of neonatal gut microflora in infants in whom atopy was and was not developing. J Allergy Clin Immunol 107, 129-134.

397. Rautava S, Ruuskanen O, Ouwehand A, et al. (2004) The hygiene hypothesis of atopic disease-an extended version. J Pediatr Gastroenterol Nutr 38, 378-388.

398. Sudo N, Sawamura S, Tanaka K, et al. (1997) The requirement of intestinal bacterial flora for the development of an $\mathrm{IgE}$ production system fully susceptible to oral tolerance induction. J Immunol 159, 1739-1745.

399. Macfarlane S \& Macfarlane GT (2003) Food and the large intestine. In Gut Flora, Nutrition, Immunity and Health, $[R$ Fuller and $G$ Perdigon, editors]. Oxford: Blackwell Publishing.

400. Gibson GR \& Roberfroid MB (1995) Dietary modulation of the human colonic microbiota: introducing the concept of prebiotics. J Nutr 125, 1401-1412.

401. Fuller R \& Gibson GR (1997) Modification of the intestinal microflora using probiotics and prebiotics. Scand J Gastroenterol Suppl 222, 28-31.

402. Fooks LJ, Fuller R \& Gibson GR (1999) Prebiotics, probiotics and human gut microbiology. Int Dairy J 9, 53-61.

403. van Loo J (2006) Inulin-type fructans as prebiotics. In Prebiotics: Development \& Application, pp. 57-100 [GR Gibson and RA Rastall, editors]. Chichester: John Wiley \& Sons.

404. van Loo J, Coussement P, de Leenheer L, et al. (1995) On the presence of inulin and oligofructose as natural ingredients in the western diet. Crit Rev Food Sci Nutr 35, 525-552.

405. Rastall RA (2006) Galacto-oligosaccharides as prebiotics. In Prebiotics: Development \& Application, pp. 101-110 [GR Gibson and RA Rastall, editors]. Chichester: John Wiley \& Sons.

406. Videla S, Vilaseca J, Antolin M, et al. (2001) Dietary inulin improves distal colitis induced by dextran sodium sulfate in the rat. Am J Gastroenterol 96, 1486-1493.

407. Schultz M, Munro K, Tannock GW, et al. (2004) Effects of feeding a probiotic preparation (SIM) containing inulin on the severity of colitis and on the composition of the intestinal microflora in HLA-B27 transgenic rats. Clin Diagn Lab Immunol 11, 581-587.

408. Hoentjen F, Welling GW, Harmsen HJ, et al. (2005) Reduction of colitis by prebiotics in HLA-B27 transgenic rats is associated with microflora changes and immunomodulation. Inflamm Bowel Dis 11, 977-985

409. Femia AP, Luceri C, Dolara P, et al. (2002) Antitumorigenic activity of the prebiotic inulin enriched with oligofructose in combination with the probiotics Lactobacillus rhamnosus and Bifidobacterium lactis on azoxymethane-induced colon carcinogenesis in rats. Carcinogenesis 23, 1953-1960.

410. Saavedra JM, Tschernia A, Moore N, et al. (1999) Gastrointestinal function in infants consuming a weaning food supplemented with oligo-fructose, a prebiotic. $J$ Pediatr Gastroenterol Nutr 29, A95.

411. Waligora-Dupriet AJ, Campeotto F, Nicolis I, et al. (2007) Effect of oligofructose supplementation on gut microflora and well-being in young children attending a day care centre. Int J Food Microbiol 113, 108-113.

412. Moro G, Arslanoglu S, Stahl B, et al. (2006) A mixture of prebiotic oligosaccharides reduces the incidence of atopic dermatitis during the first six months of age. Arch Dis Child 91, 814-819.

413. Welters CF, Heineman E, Thunnissen FB, et al. (2002) Effect of dietary inulin supplementation on inflammation of pouch mucosa in patients with an ileal pouch-anal anastomosis. Dis Colon Rectum 45, 621-627.

414. Casellas F, Borruel N, Torrejon A, et al. (2007) Oral oligofructose-enriched inulin supplementation in acute ulcerative colitis is well tolerated and associated with lowered faecal calprotectin. Aliment Pharmacol Ther 25, 1061-1067.

415. FAO-WHO (2001) Joint FAO/WHO Expert Consultation on Health and Nutritional Properties of Probiotics in Food including Powder Milk with Live Lactic Acid Bacteria

416. Bergonzelli GE, Granato D, Pridmore RD, et al. (2006) GroEL of Lactobacillus johnsonii La1 (NCC 533) is cell surface associated: potential role in interactions with the host and the gastric pathogen Helicobacter pylori. Infect Immun 74, 425-434.

417. Buck BL, Altermann E, Svingerud T, et al. (2005) Functional analysis of putative adhesion factors in Lactobacillus acidophilus NCFM. Appl Environ Microbiol 71, 8344-8351.

418. Penner R, Fedorak RN \& Madsen KL (2005) Probiotics and nutraceuticals: non-medicinal treatments of gastrointestinal diseases. Curr Opin Pharmacol 5, 596-603.

419. Petrof EO, Kojima K, Ropeleski MJ, et al. (2004) Probiotics inhibit nuclear factor-kappaB and induce heat shock proteins in colonic epithelial cells through proteasome inhibition. Gastroenterology 127, 1474-1487.

420. Cario E, Gerken G \& Podolsky DK (2004) Toll-like receptor 2 enhances ZO-1-associated intestinal epithelial barrier integrity via protein kinase C. Gastroenterology 127, 224-238.

421. Neish AS, Gewirtz AT, Zeng H, et al. (2000) Prokaryotic regulation of epithelial responses by inhibition of IkappaB-alpha ubiquitination. Science 289, 1560-1563.

422. Kelly D, Campbell JI, King TP, et al. (2004) Commensal anaerobic gut bacteria attenuate inflammation by regulating nuclear-cytoplasmic shuttling of PPAR-gamma and RelA. Nat Immunol 5, 104-112.

423. O’Hara AM, O'Regan P, Fanning A, et al. (2006) Functional modulation of human intestinal epithelial cell responses by Bifidobacterium infantis and Lactobacillus salivarius. Immunology 118, 202-215.

424. Ruiz PA, Hoffmann M, Szcesny S, et al. (2005) Innate mechanisms for Bifidobacterium lactis to activate transient pro-inflammatory host responses in intestinal epithelial cells after the colonization of germ-free rats. Immunology 115, 441-450.

425. Miettinen M, Lehtonen A, Julkunen I, et al. (2000) Lactobacilli and streptococci activate NF-kappa B and STAT signaling pathways in human macrophages. J Immunol 164, 3733-3740.

426. Matsuguchi T, Takagi A, Matsuzaki T, et al. (2003) Lipoteichoic acids from Lactobacillus strains elicit strong tumor necrosis factor alpha-inducing activities in macrophages through Tolllike receptor 2. Clin Diagn Lab Immunol 10, 259-266. 
427. Smits HH, Engering A, van der Kleij D, et al. (2005) Selective probiotic bacteria induce IL-10-producing regulatory $\mathrm{T}$ cells in vitro by modulating dendritic cell function through dendritic cell-specific intercellular adhesion molecule 3-grabbing nonintegrin. J Allergy Clin Immunol 115, 1260-1267.

428. Hahm KB, Im YH, Lee C, et al. (2000) Loss of TGF-beta signaling contributes to autoimmune pancreatitis. J Clin Invest 105, 1057-1065.

429. Monteleone G, Kumberova A, Croft NM, et al. (2001) Blocking Smad7 restores TGF-betal signaling in chronic inflammatory bowel disease. J Clin Invest 108, 601-609.

430. Di Giocinto C, Marinaro M, Sanchez M, et al. (2005) Probiotics ameliorate recurrent Th1-mediated murine colitis by inducing IL-10 and IL-10-dependent TGF-beta-bearing regulatory cells. J Immunol 174, 3237-3246.

431. Fujii T, Ohtsuka Y, Lee T, et al. (2006) Bifidobacterium breve enhances transforming growth factor betal signaling by regulating Smad7 expression in preterm infants. J Pediatr Gastroenterol Nutr 43, 83-88.

432. Mimura T, Rizzello F, Helwig U, et al. (2004) Once daily high dose probiotic therapy (VSL\#3) for maintaining remission in recurrent or refractory pouchitis. Gut 53, 108-114.

433. Kuisma J, Mentula S, Jarvinen H, et al. (2003) Effect of Lactobacillus rhamnosus GG on ileal pouch inflammation and microbial flora. Aliment Pharmacol Ther 17, 509-515.

434. Gupta P, Andrew H, Kirschner BS, et al. (2000) Is Lactobacillus GG helpful in children with Crohn's disease? Results of a preliminary, open-label study. J Pediatr Gastroenterol Nutr 31, 453-457.

435. Schultz M, Timmer A, Herfarth HH, et al. (2004) Lactobacillus $\mathrm{GG}$ in inducing and maintaining remission of Crohn's disease. BMC Gastroenterol 4, 5.

436. Prantera C, Scribano ML, Falasco G, et al. (2002) Ineffectiveness of probiotics in preventing recurrence after curative resection for Crohn's disease: a randomised controlled trial with Lactobacillus GG. Gut 51, 405-409.

437. Marteau P, Lemann M, Seksik P, et al. (2006) Ineffectiveness of Lactobacillus johnsonii LA1 for prophylaxis of postoperative recurrence in Crohn's disease: a randomised, double blind, placebo controlled GETAID trial. Gut 55, 842-847.

438. Van Gossum A, Dewit O, Louis E, et al. (2007) Multicenter randomized-controlled clinical trial of probiotics (Lactobacillus johnsonii, LA1) on early endoscopic recurrence of Crohn's disease after lleo-caecal resection. Inflamm Bowel Dis 13, 135-142.

439. Kruis W, Fric P, Pokrotnieks J, et al. (2004) Maintaining remission of ulcerative colitis with the probiotic Escherichia coli Nissle 1917 is as effective as with standard mesalazine. Gut 53, 1617-1623.

440. Rembacken BJ, Snelling AM, Hawkey PM, et al. (1999) Non-pathogenic Escherichia coli versus mesalazine for the treatment of ulcerative colitis: a randomised trial. Lancet 354, 635-639.

441. Tursi A, Brandimarte G, Giorgetti GM, et al. (2004) Low-dose balsalazide plus a high-potency probiotic preparation is more effective than balsalazide alone or mesalazine in the treatment of acute mild-to-moderate ulcerative colitis. Med Sci Monit 10, I126-I131.

442. Zocco MA, dal Verme LZ, Cremonini F, et al. (2006) Efficacy of Lactobacillus GG in maintaining remission of ulcerative colitis. Aliment Pharmacol Ther 23, 1567-1574.
443. Cui HH, Chen CL, Wang JD, et al. (2004) Effects of probiotic on intestinal mucosa of patients with ulcerative colitis. World $J$ Gastroenterol 10, 1521-1525.

444. Ishikawa H, Akedo I, Umesaki Y, et al. (2003) Randomized controlled trial of the effect of bifidobacteria-fermented milk on ulcerative colitis. J Am Coll Nutr 22, 56-63.

445. Rovensky J, Svik K, Stancikova M, et al. (2002) Treatment of experimental adjuvant arthritis with the combination of methotrexate and lyophilized Enterococcus faecium enriched with organic selenium. Folia Microbiol (Praha) 47, $573-578$.

446. Hatakka K, Martio J, Korpela M, et al. (2003) Effects of probiotic therapy on the activity and activation of mild rheumatoid arthritis - a pilot study. Scand J Rheumatol 32, 211-215.

447. Shida K, Makino K, Morishita A, et al. (1998) Lactobacillus casei inhibits antigen-induced IgE secretion through regulation of cytokine production in murine splenocyte cultures. Int Arch Allergy Immunol 115, 278-287.

448. Matsuzaki T, Yamazaki R, Hashimoto S, et al. (1998) The effect of oral feeding of Lactobacillus casei strain Shirota on immunoglobulin E production in mice. J Dairy Sci 81, 48-53.

449. Murosaki S, Yamamoto Y, Ito K, et al. (1998) Heat-killed Lactobacillus plantarum L-137 suppresses naturally fed antigen-specific IgE production by stimulation of IL-12 production in mice. J Allergy Clin Immunol 102, 57-64.

450. Shida K, Takahashi R, Iwadate E, et al. (2002) Lactobacillus casei strain Shirota suppresses serum immunoglobulin E and immunoglobulin G1 responses and systemic anaphylaxis in a food allergy model. Clin Exp Allergy 32, 563-570.

451. Björkstén B, Sepp E, Julge K, et al. (2001) Allergy development and the intestinal microflora during the first year of life. J Allergy Clin Immunol 108, 516-520.

452. Ouwehand AC, Isolauri E, He F, et al. (2001) Differences in Bifidobacterium flora composition in allergic and healthy infants. J Allergy Clin Immunol 108, 144-145.

453. Majamaa H \& Isolauri E (1997) Probiotics: a novel approach in the management of food allergy. J Allergy Clin Immunol 99, 179-185.

454. Isolauri E, Arvola T, Sütas Y, et al. (2000) Probiotics in the management of atopic eczema. Clin Exp Allergy 30, 1604-1610.

455. Kalliomäki M, Salminen S, Arvilommi H, et al. (2001) Probiotics in primary prevention of atopic disease: a randomised placebo-controlled trial. Lancet 357, 1076-1079.

456. Kalliomäki M, Salminen S, Poussa T, et al. (2003) Probiotics and prevention of atopic disease: 4-year follow-up of a randomised placebo-controlled trial. Lancet 361, 1869-1871.

457. Rosenfeldt V, Benfeldt E, Nielsen SD, et al. (2003) Effect of probiotic Lactobacillus strains in children with atopic dermatitis. J Allergy Clin Immunol 111, 389-395.

458. Hlivak P, Odraska J, Ferencik M, et al. (2005) One-year application of probiotic strain Enterococcus faecium M-74 decreases serum cholesterol levels. Bratisl Lek Listy 106, 67-72.

459. Naruszewicz M, Johansson ML, Zapolska-Downar D, et al. (2002) Effect of Lactobacillus plantarum 299v on cardiovascular disease risk factors in smokers. Am J Clin Nutr 76, 1249-1255.

460. Bach JF (2002) The effect of infections on susceptibility to autoimmune and allergic diseases. New Engl J Med 347, 911-920. 